Florida International University FIU Digital Commons

$11-23-2016$

\title{
Optical Coherence Photoacoustic Microscopy (OC-PAM) for Multimodal Imaging
}

Xiaojing Liu

Florida International University, xliu031@fiu.edu

DOI: $10.25148 /$ etd.FIDC001785

Follow this and additional works at: https://digitalcommons.fiu.edu/etd

Part of the Bioimaging and Biomedical Optics Commons, and the Ophthalmology Commons

\section{Recommended Citation}

Liu, Xiaojing, "Optical Coherence Photoacoustic Microscopy (OC-PAM) for Multimodal Imaging" (2016). FIU Electronic Theses and Dissertations. 3189.

https://digitalcommons.fiu.edu/etd/3189 


\title{
FLORIDA INTERNATIONAL UNIVERSITY
}

Miami, Florida

\section{OPTICAL COHERENCE PHOTOACOUSTIC MICROSCOPY (OC-PAM) \\ FOR MULTIMODAL IMAGING}

\author{
A dissertation submitted in partial fulfillment of \\ the requirements for the degree of \\ DOCTOR OF PHILOSOPHY
}

in

BIOMEDICAL ENGINEERING

by

Xiaojing Liu

2017 
To: $\quad$ Interim Dean Ranu Jung College of Engineering and Computing

This dissertation, written by Xiaojing Liu, and entitled Optical Coherence Photoacoustic Microscopy (OC-PAM) for Multimodal Imaging, having been approved in respect to style and intellectual content, is referred to you for judgment.

We have read this dissertation and recommend that it be approved.

$\begin{array}{r}\text { Richard A. Bone } \\ \hline \text { Chenzhong Li } \\ \hline \text { Jessica Ramella-Roman } \\ \hline \text { Shuliang Jiao, Major Professor }\end{array}$

Date of Defense: November 23, 2016

The dissertation of Xiaojing Liu is approved.

Interim Dean Ranu Jung College of Engineering and Computing

Andrés G. Gil

Vice President for Research and Economic Development and Dean of the University Graduate School

Florida International University, 2017 
Portion of Chapter 4 (C IOP Publishing. Reproduced with permission. All rights

reserved. https://doi.org/10.1088/2040-8978/18/6/064001

All other materials (C) Copyright 2017 by Xiaojing Liu 


\section{DEDICATION}

I dedicate this dissertation to my father, Zhengyi Liu and my mother, Xiangdong Li. Their unconditional support, encouragement, and selfless love have made this journey possible. 


\section{ACKNOWLEDGMENTS}

I would like to express my special appreciation and thanks to my major professor Dr. Shuliang Jiao. This dissertation could not have been completed without the great support that I have received from Dr. Jiao. His mentorship enlightened my view of biomedical research and assisted me to overcome the difficulties in my studies and my life. I would also like to give thanks to my committee members, Dr. Richard A. Bone, Dr. Chenzhong Li, Dr. Wei-Chiang Lin and Dr. Jessica Ramella-Roman, for all the help and support I received from them. I would like to thank all my lab members, Dr. Tan Liu, Dr. Cuixia Dai, Dr. Minshan Jiang, Daniela Rodriguez, Aime Ruiz, and Zahra Nafar for their assistance and support in the research projects. As a freshman in this lab four years ago, I received tremendous help and advice from all the past lab members and that's the foundation of my research career. I would also like to acknowledge the assistance provided by Dr. Rong Wen and Dr. Yiwen Li from Bascom Palmer Eye Institute for performing histological analysis on rat eyes and advising how to do tail vein injection of nanorods successfully.

I would like to acknowledge the financial support received throughout my Ph.D. study, including the Teaching Assistantship and Research Assistantship from the Department of Biomedical Engineering and Research Assistantship from National Institute of Health grants to Dr. Shuliang Jiao.

My gratitude also goes to all my friends and colleagues, without whom, this experience would have been incomplete. Their general help and friendship were all greatly appreciated. 
Finally my most important acknowledgments are towards my family who have been encouraging, supportive and shown belief in me and my work. I thank my parents Zhengyi Liu and Xiangdong Li, my Auntie Lisa and my whole family for their unconditional love and support, which have kept me going through the hard times. 
ABSTRACT OF THE DISSERTATION

OPTICAL COHERENCE PHOTOACOUSTIC MICROSCOPY (OC-PAM)

FOR MULTIMODAL IMAGING

by

Xiaojing Liu

Florida International University, 2017

Miami, Florida

Professor Shuliang Jiao, Major Professor

Optical coherence tomography (OCT) and Photoacoustic microscopy (PAM) are two noninvasive, high-resolution, three-dimensional, biomedical imaging modalities based on different contrast mechanisms. OCT detects the light backscattered from a biological sample either in the time or spectral domain using an interferometer to form an image. PAM is sensitive to optical absorption by detecting the light-induced acoustic waves to form an image. Due to their complementary contrast mechanisms, OCT and PAM are suitable for being combined to achieve multimodal imaging.

In this dissertation, an optical coherence photoacoustic microscopy (OC-PAM) system was developed for in vivo multimodal retinal imaging with a pulsed broadband NIR light source. To test the capabilities of the system on multimodal ophthalmic imaging, the retina of pigmented rats was imaged. The OCT images showed the retinal structures with quality similar to conventional OCT, while the PAM images revealed the distribution of melanin in the retina since the NIR PAM signals are generated mainly from melanin in the posterior segment of the eye.

By using the pulsed broadband light source, the OCT image quality highly depends on the pulse-to-pulse stability of the light source without averaging. In addition, laser safety is always a 
concern for in vivo applications, especially for eye imaging with a pulsed light source. Therefore, a continuous wave (CW) light source is desired for OC-PAM applications. An OC-PAM system using an intensity-modulated CW superluminescent diode was then developed. The system was tested for multimodal imaging the vasculature of a mouse ear in vivo by using Gold Nanorods (GNRs) as contrast agent for PAM, as well as excised porcine eyes ex vivo.

Since the quantitative information of the optical properties extracted from the proposed NIR OC-PAM system is potentially able to provide a unique technique to evaluate the existence of melanin and lipofuscin specifically, a phantom study has been conducted and the relationship between image intensity of OCT and PAM was interpreted to represent the relationship between the optical scattering property and optical absorption property. It will be strong evidence for practical application of the proposed NIR OC-PAM system. 


\section{TABLE OF CONTENTS}

CHAPTER

PAGE

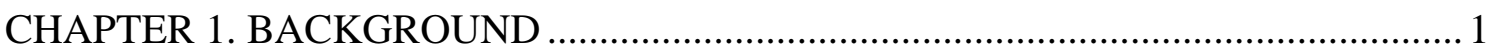

1.1 Optical Coherence Tomography (OCT) ......................................................... 1

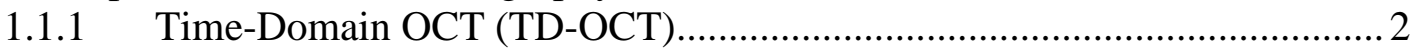

1.1.2 Spectral-Domain OCT (SD-OCT) ……………....................................... 4

1.2 Photoacoustic Microscopy (PAM) ................................................................... 6

1.2.1 Acoustic-Resolution PAM (AR-PAM) …………………………………. 7

1.2.2 Optical-Resolution PAM (OR-PAM) ....................................................... 7

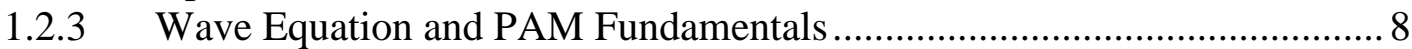

1.3 Combination of OCT and PAM ..................................................................

1.4 Optical Coherence Photoacoustic Microscopy (OC-PAM) …………………...... 10

1.5 Significance and Aims of the Study ............................................................... 11

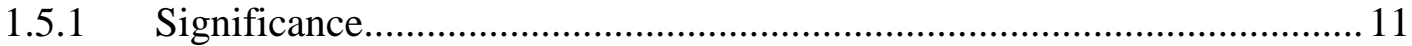

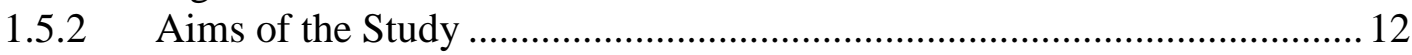

\section{CHAPTER 2. EFFECT OF CONTACT LENS ON OPTICAL COHERENCE} TOMOGRAPHY IMAGING OF RODENT RETINA.................................................... 14

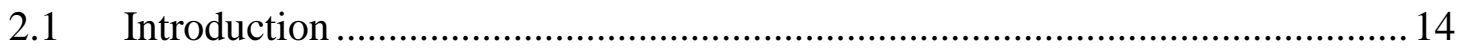

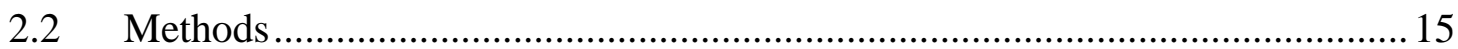

2.2.1 Experimental System .......................................................................... 15

2.2.2 Animal Imaging ................................................................................ 16

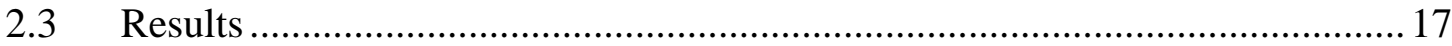

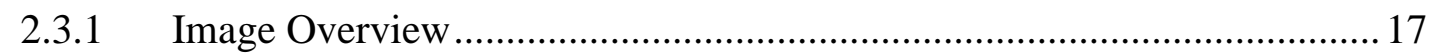

2.3.2 Spatial Resolution .......................................................................... 19

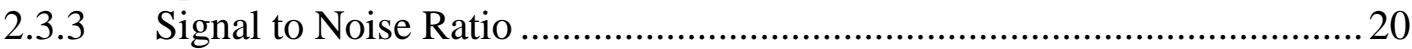

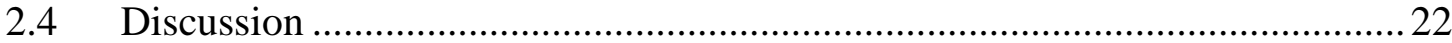

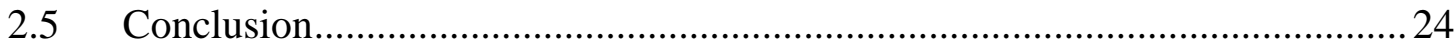

CHAPTER 3. OPTICAL COHERENCE PHOTOACOUSTIC MICROSCOPY FOR IN VIVO MULTIMODAL RETINAL IMAGING ………….............................................25

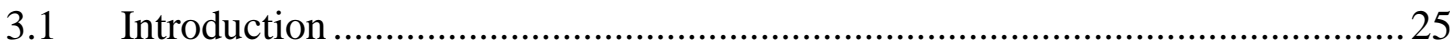

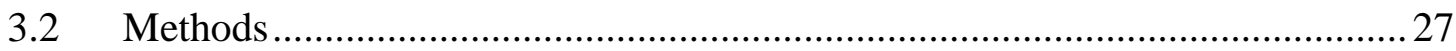

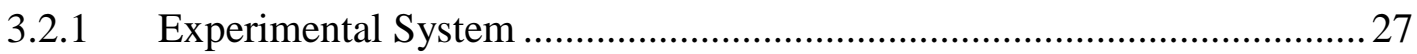

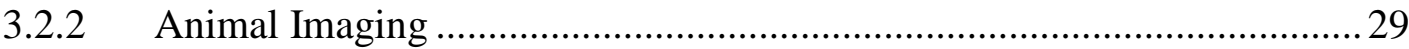

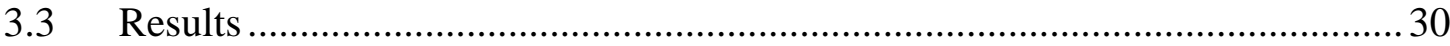

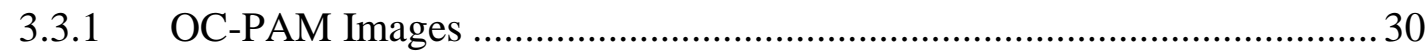

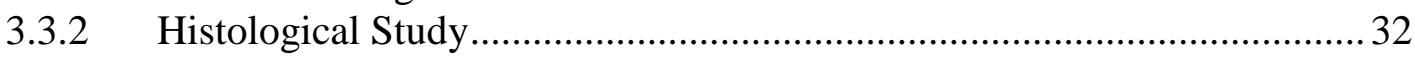

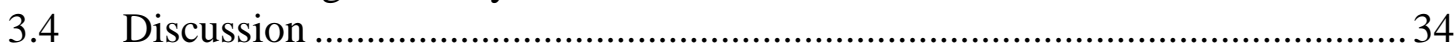

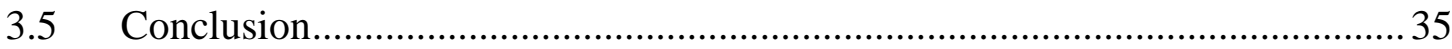




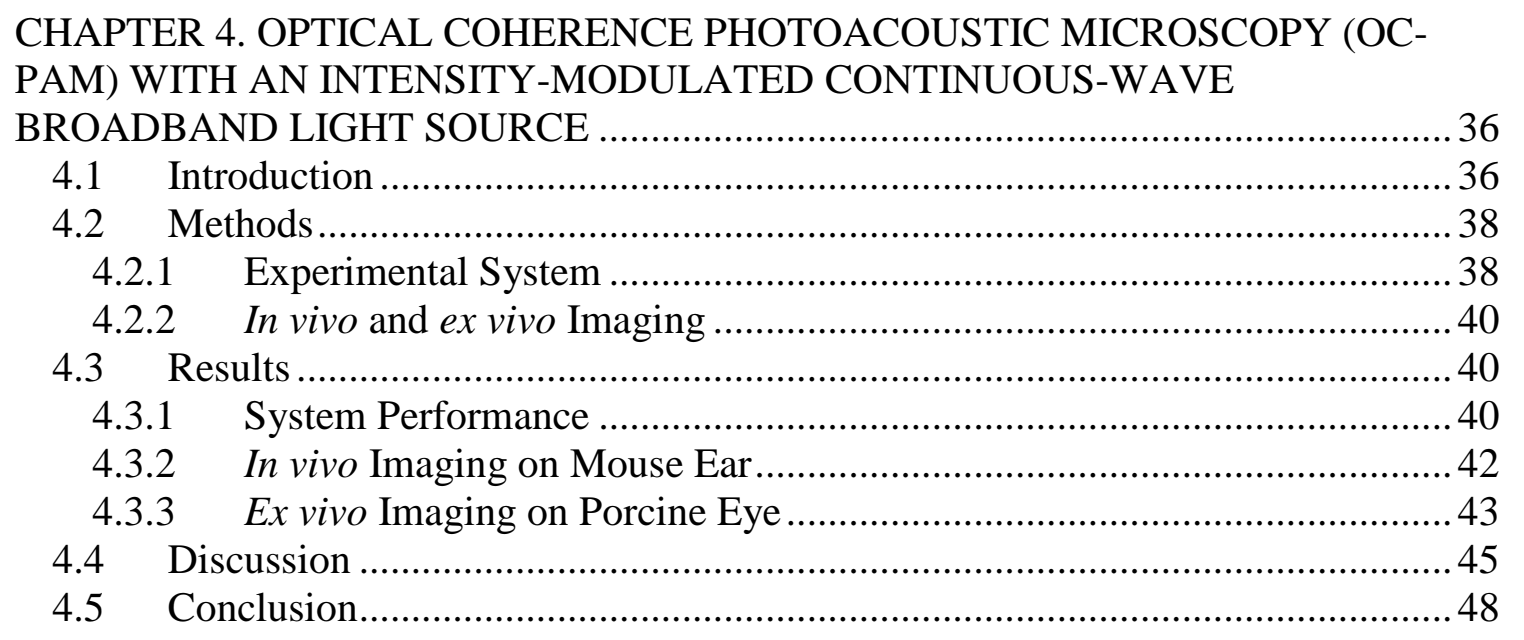

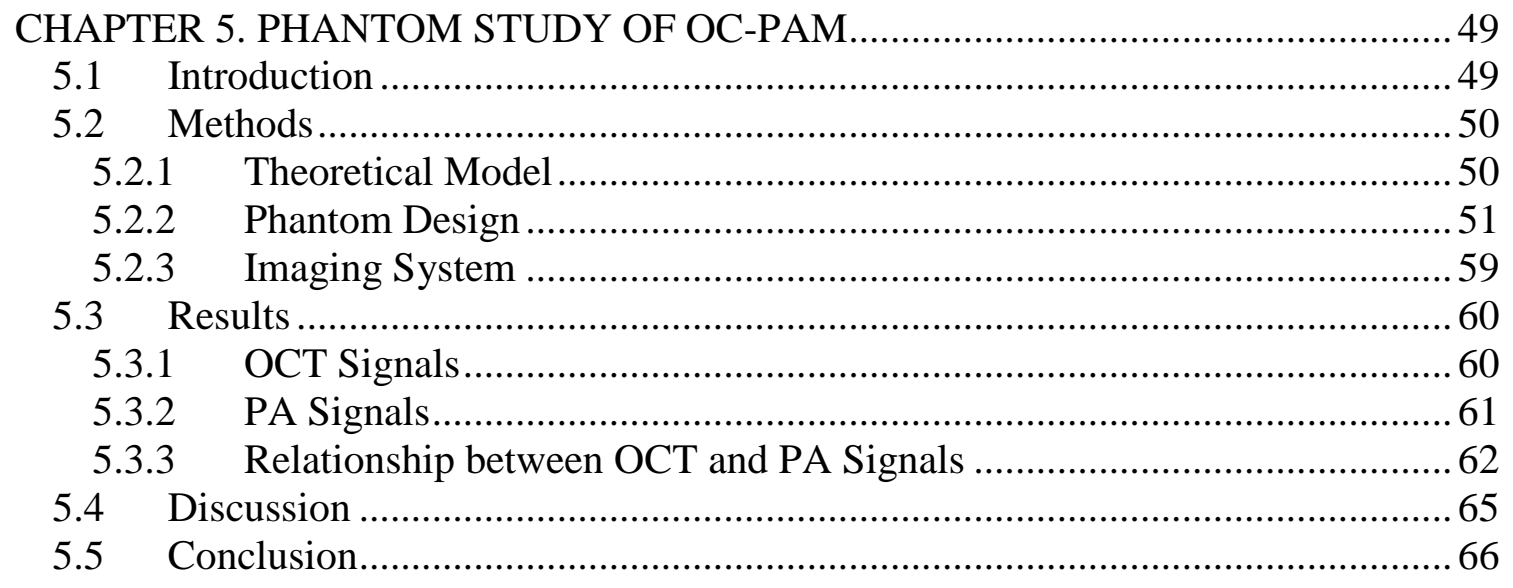

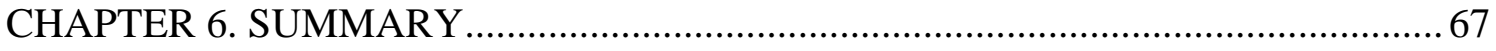

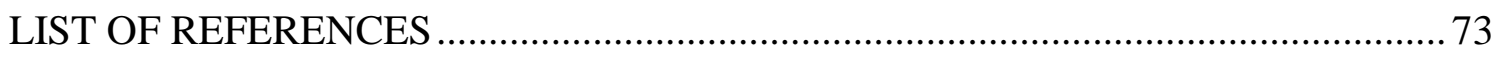

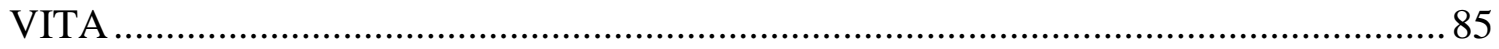




\section{LIST OF TABLES}

TABLE

PAGE

Table 2.1 SNR Calculation of OCT A-lines with and without contact lens ................... 21

Table 2.2 Summarized information of rats shown in Figure 2.6 ................................. 24

Table 5.1 Concentration of Intralipid and NIR dye in the phantom solution ..................58

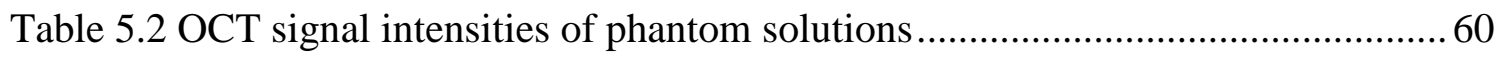

Table 5.3 PA signal intensities of phantom solutions............................................61

Table 5.4 Ratio of OCT signal intensities to PA signal intensities of phantom

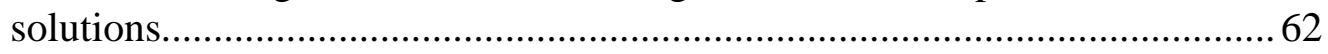




\section{LIST OF FIGURES}

FIGURE

PAGE

Figure 1.1 Schematic of Time-Domain OCT …....................................................... 2

Figure 2.1 Schematic of the experimental OCT system. M: Mirror; C1, C2:

Collimator; L1, L2: Lens; PC: Polarization controller.

Figure 2.2 OCT fundus images generated from the acquired 3D OCT datasets for the retina of rat 1 (a and b), rat 2 (c and d) and rat 3 (e and f), with (b, d, f) and without (a, c, e) contact lens, respectively. (Bar: $150 \mu \mathrm{m})$

Figure 2.3 Two pairs of OCT images for the retina of two rats with and without contact lens. (a) and (b): OCT images of rat 1 without and with contact lens. (c) and (d): OCT images of rat 2 without and with contact lens. The images consist of 2048 A-lines. (Bar: $200 \mu \mathrm{m}$ ).

Figure 2.4 Blood vessel profiles generated by summation of the OCT signal along the depth direction. (a) The profile of blood vessel 1 in the area marked red in Figure 2.3 (a-b); (b) The profile of blood vessel 2 in the area marked yellow in Figure 2.3(c-d).

Figure 2.5 OCT signals at the same location of the retina chosen from a pair of OCT images with and without contact lens, the locations of which are marked as white lines in Figure 2(a) and Figure 2(b). (c) OCT signal without contact lens at the location marked in (b). (Bar: $200 \mu \mathrm{m})$

Figure 2.6 Typical OCT images of rat's cornea. (a)-(c) Cornea images of Sprague Dawley rats in different ages, the red arrows indicate the location of cornea defects. (d) and (e) Cornea images of Long Evans rats in different ages, the red arrows indicate the location of cornea defects. (f) The rat's cornea with contact lens, the white arrow indicates the front surface of contact lens and red arrow shows the interface between rear surface of contact lens and rat's cornea. The images consist of 2048 A-lines.

Figure 3.1 Schematic of the experimental system of a fiber-based OC-PAM. L1, L2: lens; PC: polarization controller; UT: ultrasonic transducer; M1, M2: mirror; C1, C2: collimator.

Figure 3.2 Performance of the OCT mode. (a) The measured spectrum of the light source. (b) The calculated PSF of the OCT subsystem.

Figure 3.3 The simultaneously acquired OCT and PAM images of two rat eyes. (a) and (c): OCT fundus images generated from the acquired 3D OCT 
datasets. (b) and (d): The maximum-amplitude-projection (MAP) of the PA datasets. Bar: $200 \mu \mathrm{m}$

Figure 3.4 The simultaneously acquired OCT and PAM B-scan images. The red arrow indicates retinal blood vessel. Bar: $100 \mu \mathrm{m}$

Figure 3.5 Comparison of the histologic image (a) with the OCT cross-sectional image (b) of rat retina. Bar: $100 \mu \mathrm{m}$. (c-e) Additional histologic images with different areas of $593 \times 444 \mu \mathrm{m}^{2}, 213 \times 213 \mu \mathrm{m}^{2}$ and $195 \times 147 \mu \mathrm{m}^{2}$, respectively

Figure 4.1 Schematic of the experimental system of a fiber-based OC-PAM with an intensity-modulated light source. L1, L2, L3: achromatic lens; PC: polarization controller; UT: ultrasonic transducer; M1, M2: mirror; C1, C2: collimator.

Figure 4.2 Performance of the OCT. (a) The measured spectrum of the light source. (b) The calculated PSF of the OCT subsystem.

Figure 4.3 Images of USAF 1951 resolution target. (a) Microscopic image. (b) Zoomed-in microscopic image of the scanned area. (c) PA image. (d) OCT projection image generated from the acquired 3D OCT dataset..

Figure 4.4 Simultaneously acquired PAM and OCT images of a mouse ear in vivo with GNRs injection. (a) PA image. (b) OCT projection images generated from the acquired 3D OCT dataset. (c) OCT cross-sectional image, whose location is marked as a white line on (a) and (b). Bar: $300 \mu \mathrm{m}(\mathrm{a}, \mathrm{b}) 180 \mu \mathrm{m}$ (c)

Figure 4.5 Simultaneously acquired PAM and OCT images of a porcine eye ex vivo. (a) and (c) PA images. (b) and (d) OCT projection images generated from the acquired 3D OCT datasets. Bar: Bar: $150 \mu \mathrm{m}(\mathrm{a}, \mathrm{b}) 300 \mu \mathrm{m}(\mathrm{c}, \mathrm{d})$

Figure 4.6 Comparison of OCT cross-sectional image and histologic image of porcine eye ex vivo. (a) PA image. (b) OCT projection image, where the white line marks the location of the OCT cross-sectional image shown in (d). (c) Histologic image of porcine eye. (d) OCT cross-sectional image of porcine eye. Bar: $150 \mu \mathrm{m}(\mathrm{a}, \mathrm{b}) 50 \mu \mathrm{m}$ (c) $90 \mu \mathrm{m}(\mathrm{d})$.

Figure 4.7 Comparison of OCT cross-sectional images acquired by OCT system using pulsed broadband light source and cw light source, respectively. (a) OCT cross-sectional images using pulse broadband light source (b) OCT cross-sectional images using cw light source.

Figure 5.1 The absorption coefficient of Intralipid-10\%................................................52 
Figure 5.2 The scattering properties of Intralipid-10\%. (a) The scattering coefficient of Intralipid-10\%. (b) The anisotropy of scattering of Intralipid-10\%.........5 52

Figure 5.3 The reduced scattering coefficient of Intralipid-10\%................................53

Figure 5.4 The absorption spectrum of NIR869A. ................................................... 54

Figure 5.5 The absorption coefficient of melanosome. ............................................ 55

Figure 5.6 OCT signal intensities of pilot phantom solutions ....................................56

Figure 5.7 PA signal intensities of pilot phantom solutions ......................................56

Figure 5.8 Ratio of OCT signal intensities to PA signal intensities of pilot phantom

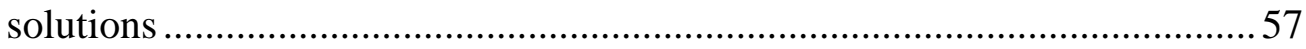

Figure 5.9 Photos of the phantom solutions prepared based on the mix ratio in Table 5.1. (a) Group A with 2\% Intralipid. (b) Group B with 8\% Intralipid. (c) Group C with 14\% Intralipid. (d) Group D with 20\% Intralipid. In each picture, the ratio of the reduced scattering coefficient to the absorption coefficient is $0.3,0.4,0.45,0.5,0.55$ and 0.6 from left to right. ..................58

Figure 5.10 OCT signal intensities of phantom solutions ........................................60

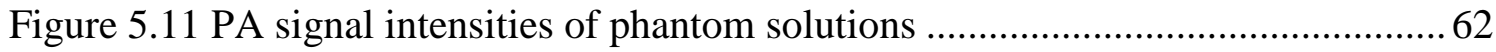

Figure 5.12 Ratio of OCT signal intensities to PA signal intensities of phantom solutions

Figure 5.13 Mean and standard deviation of the ratio of OCT signal intensities to PA signal intensities of phantom solutions 


\section{CHAPTER 1. BACKGROUND}

\subsection{Optical Coherence Tomography (OCT)}

Optical coherence tomography $(\mathrm{OCT})[1,2]$ is a low-coherence, interferometry-based, threedimensional imaging modality. In OCT, the contrasts are provided by the photons backscattered from the biological tissues, which carry information about mainly their scattering properties. OCT can thus provide imaging of the micro-structures of biological tissues like the layered structures of the retina.

OCT was first reported by Huang et al.[1] in 1991 and demonstrated to produce a twodimensional image of microstructure in retina and coronary artery in vitro. Since then, the development of OCT has greatly changed the landscape of diagnosis and patient care in ophthalmology. In vivo measurements of human retinal structure with OCT was first described by Swanson et al. in 1993.[3] Hee et al. demonstrated its capability of quantitative assessment of retinal thickness with high resolution in patients with macular edema[4]. They also used OCT to study the morphology of eye with age-related macular degeneration (AMD) and Choroidal neovascularization (CNV).[5] First commercialized OCT was licensed to Carl Zeiss Meditec, Incorporated for ophthalmic applications in 1996. OCT has been used to evaluate posterior vitreous detachment caused by aging[6], and it also improved understanding of pathogenesis, staging, and management of macular hole[7]. Medeiros et al. has demonstrated the ability of OCT to differentiate between healthy eyes and eyes with glaucoma by measuring the retinal nerve fiber layer (RNFL), the optic nerve head, and macular thickness parameters.[8] OCT has also been used for measurement and imaging of water concentration in the human cornea in vitro.[9] Furthermore, several studies have used OCT to evaluate the efficacy and safety of different therapies for ocular diseases.[10-12] 
Besides ophthalmology, the clinical applications of OCT have also been demonstrated in a variety of areas, including dermatology[13, 14], cardiology[15-20], gastroenterology[21-24], oncology[25, 26] and more[27-30]. Currently, more research on OCT has been focus on the improvement of light source, bean delivery instruments, detection schemes and multimodal application so as to improve imaging quality, imaging speed and imaging contrast.

\subsubsection{Time-Domain OCT (TD-OCT)}

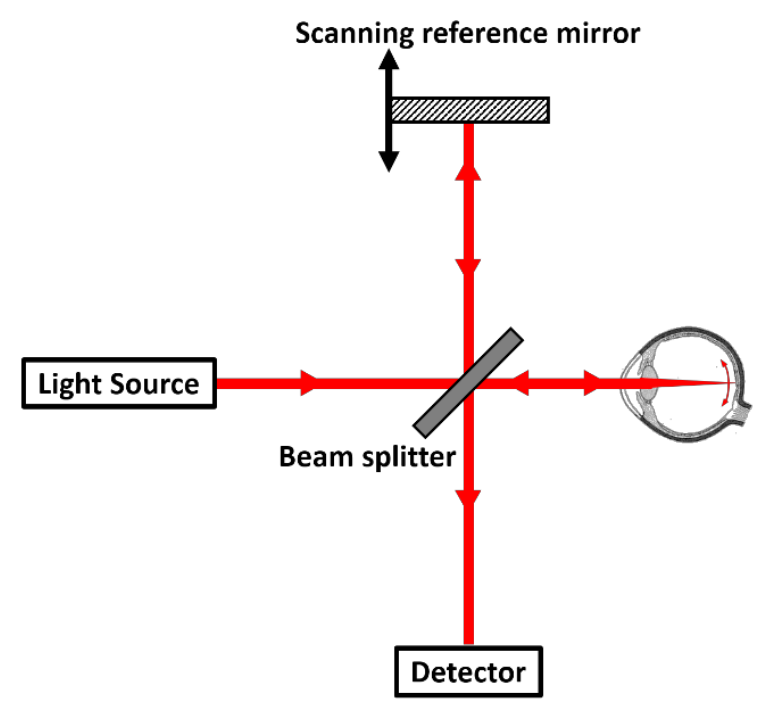

Figure 1.1 Schematic of Time-Domain OCT

Conventional OCT detects the interference signal caused by the back-scattered light from sample and the reflected light from a scanning reference mirror.[1] This interference signal carries the information about the reflectivity and the location of the structure within a sample and it is detected by an interferometer to form a reflectivity profile of the sample. By using a low-coherence broadband light source, the light will only coherently interfere within its coherence length, thus the depth of the structure can be determined by the location of the scanning reference mirror. When the light is scanning laterally on the sample, a two or three- dimensional image of the reflectivity profile can be obtained. Figure 1.1 shows a schematic of conventional Time-Domain OCT. 
As a Gaussian spectral distributed broadband light source used in TD-OCT, the power density can be expressed as:

$$
G(v)=\frac{2 \sqrt{\ln 2}}{\Delta v \sqrt{\pi}} \exp \left[-\left(2 \sqrt{\ln 2} \frac{v-\bar{v}}{\Delta v}\right)^{2}\right]
$$

where $v$ is the frequency of light source, $\bar{v}$ is the center frequency, and $\Delta v$ is the bandwidth.

The complex amplitude of the electromagnetic field in the reference arm and the sample arm can be expressed as:

$$
\boldsymbol{E}_{s}=E_{S} e^{i\left(k l_{s}-\omega t+\phi_{1}\right)}, \boldsymbol{E}_{r}=E_{r} e^{i\left(k l_{r}-\omega t+\phi_{2}\right)},
$$

where $E_{s}$ and $E_{r}$ are the magnitude of the electromagnetic field at the detector reflected from the sample arm and reference arm, respectively; $\mathrm{k}$ is the free space wave number; $l_{s}$ and $l_{r}$ are the roundtrip optical path length in the sample arm and reference arm, respectively; $\phi_{1}$ and $\phi_{2}$ are the initial phase of the electromagnetic field in the sample arm and reference arm, respectively.

The light source is divided into the reference arm and sample arm by a beam splitter and the reflected light beams from the reference mirror and sample are recombined at the beam splitter. So the intensity of the light collect by detector can be expressed as[31]:

$$
\begin{aligned}
I & =|\boldsymbol{E}|^{2}=\left[\boldsymbol{E}_{s}+\boldsymbol{E}_{r}\right]\left[\boldsymbol{E}_{s}+\boldsymbol{E}_{r}\right]^{*} \\
& =\left(E_{s} e^{i\left(k l_{s}-\omega t+\phi_{1}\right)}+E_{r} e^{i\left(k l_{r}-\omega t+\phi_{2}\right)}\right) \cdot\left(E_{s} e^{i\left(k l_{s}-\omega t+\phi_{1}\right)}+E_{r} e^{i\left(k l_{r}-\omega t+\phi_{2}\right)}\right)^{*} \\
& =E_{s}{ }^{2}+E_{r}{ }^{2}+2 E_{s} E_{r} \cos \Delta \phi
\end{aligned}
$$

where the phase difference is: $\Delta \phi=k\left(l_{s}-l_{r}\right)+\left(\phi_{1}-\phi_{2}\right)=\frac{2 \pi}{\lambda}\left(l_{s}-l_{r}\right)+\Delta \phi_{0}$

Equation 1.3 can be written as:

$$
I=\frac{1}{2} P\left(R_{S}+R_{r}\right)+P \sqrt{R_{S} R_{r}} \int G(v) \cos (2 \pi v \tau) d v
$$


where $\mathrm{P}$ is the power of light source; $R_{s}$ and $R_{r}$ are the intensity reflectivity of the sample and reference mirror, respectively. In this Equation, the first term is the DC component and the second term is the interference. By using Equation 1.4, the detected interference signal can be expressed as:

$$
\begin{aligned}
\tilde{I} & =\sqrt{R_{s} R_{r}} \int \frac{2 \sqrt{\ln 2}}{\Delta v \sqrt{\pi}} \exp \left[-\left(2 \sqrt{\ln 2} \frac{v-\bar{v}}{\Delta v}\right)^{2}\right] \cos (2 \pi v \tau) d v \\
& =\sqrt{R_{s} R_{r}} \frac{\sqrt{\ln 2}}{\Delta v \sqrt{\pi}} \int \exp \left[-\left(2 \sqrt{\ln 2} \frac{v-\bar{v}}{\Delta v}\right)^{2}\right] \exp (i 2 \pi v \tau) d v \\
& =\frac{\sqrt{R_{s} R_{r}}}{2 \sqrt{2 \pi}} \exp \left[-\left(\frac{\pi \Delta v \tau}{2 \sqrt{\ln 2}}\right)^{2}\right] \cos (2 \pi \bar{v} \tau)
\end{aligned}
$$

The depth resolution can be derived by the FWHM (full width at half maximum) of the interference signal as[32]:

$$
\Delta l_{F W H M}=\frac{2 \ln 2}{\pi} \frac{\lambda_{0}^{2}}{\Delta \lambda} \approx 0.44 \frac{\lambda_{0}^{2}}{\Delta \lambda}
$$

where $\lambda_{0}$ is the center wavelength and $\Delta \lambda$ is the bandwidth of the light source.

\subsubsection{Spectral-Domain OCT (SD-OCT)}

A major limitation of TD-OCT is its relatively slow imaging speed because a mechanical scan is required in the reference arm.[33] However, in Spectral-Domain OCT the axial scan can be implemented by Fourier transforming the spectrum of light, which improves its imaging speed and sensitivity. By using a linear array detector such as a CCD camera, the spectral-domain signal reflected from a fixed reference mirror and sample arm can be detected.[33, 34] The spectral density of the combined reference and sample light can be expressed as[35]: 


$$
\begin{aligned}
G_{d}(v)=G_{s}(v)\{1 & +\sum_{n} R_{n}+2 \sum_{n \neq m} \sqrt{R_{n} R_{m}} \cos \left[2 \pi v\left(\tau_{n}-\tau_{m}\right)\right] \\
& \left.+2 \sum_{n} \sqrt{R_{n}} \cos \left[2 \pi v\left(\tau_{n}-\tau_{r}\right)\right]\right\}
\end{aligned}
$$

where $v$ is the light frequency, $R_{n}$ and $R_{m}$ are the reflectivity of the $n$th and $m$ th scatterer, respectively, the reflection of the reference arm is treated as unity, $G_{s}(v)$ is the spectral density of the light source; $\tau_{n}$ and $\tau_{m}$ are the propagation time of the light reflected by $n$th and $m$ th scatterer, respectively and $\tau_{r}$ is the propagation time of the light reflected by reference mirror.

The spectral-domain signal can be transformed to the time-domain by using the WienerKhinchin theorem[36]:

$$
\Gamma(\tau)=<E(t) E^{*}(t+\tau)>=F T^{-1}\left[G_{s}(v)\right]
$$

where $\Gamma(\tau)$ is the autocorrelation function of the light source, $E(t)$ is the amplitude of the electromagnetic field of light, $F T^{-1}$ denotes the inverse Fourier transform and the angle brackets denote integration over time. By taking inverse Fourier transformation of Equation 1.7, the timedomain intensity signal can be obtained as:

$$
\begin{aligned}
I(\tau) & =\Gamma(\tau)+\Gamma(\tau) \sum_{n} R_{n} \\
& +2 \sum_{n \neq m} \sqrt{R_{n} R_{m}} \Gamma\left[\tau \pm 2\left(\tau_{n}-\tau_{m}\right)\right]+2 \sum_{n} \sqrt{R_{n}} \Gamma\left[\tau \pm 2\left(\tau_{n}-\tau_{r}\right)\right]
\end{aligned}
$$

In Equation 1.6 and 1.8, the third terms are the mutual interference for light scattered within the sample and the last terms are the interference between sample and reference arm, which can be used to derive the reflectivity profile of the sample.

The depth resolution of SD-OCT is the same as TD-OCT: 


$$
\Delta l_{F W H M}=\frac{2 \ln 2}{\pi} \frac{\lambda_{0}^{2}}{\Delta \lambda} \approx 0.44 \frac{\lambda_{0}^{2}}{\Delta \lambda}
$$

where $\lambda_{0}$ is the center wavelength and $\Delta \lambda$ is the bandwidth of the light source.

\subsection{Photoacoustic Microscopy (PAM)}

Photoacoustic microscopy (PAM)[37-41] is a novel microscopic three-dimensional noninvasive imaging modality that is used for imaging the microvasculature and the associated blood oxygenation of biological tissues[42]. PAM is based on the optical-absorption properties of biological tissues. When irradiated by a short laser pulse, the optical energy absorbed by a substance, such as hemoglobin or melanin in tissue, is converted to heat, which induces localized thermo-elastic expansion and leads to the generation of wideband ultrasonic waves. The ultrasonic waves can be detected with an ultrasonic transducer to reconstruct an image mapping the location and absorption strength of the absorbers.

The photoacoustic effect was first reported by Alexander Graham Bell in 1880[43] while its application in biomedical area began in the 1970s[44]. Since then, the progress of photoacoustic technique was very slow until a reconstruction algorithm was demonstrated and photoacoustic tomography (PAT) was developed in 1995.[45, 46] In vivo functional imaging of rat brains by PAT was reported in 2003.[47] With the rapid development of PAT in the past decade, the applications of PAT have been demonstrated in a variety of areas, including dermatology[48, 49], cardiology[50, 51], gastroenterology[52, 53], oncology[54, 55], neurology[47, 56, 57] and ophthalmology[39, 58].

Currently, major PAT modalities can be classified into: photoacoustic computed tomography (PACT) [59-61], photoacoustic microscopy (PAM) [38, 62] and photoacoustic endoscopy (PAE) [50-53]. In PAM, focused laser excitation or focused ultrasonic detection is implemented to achieve 
better sensitivity. The axial resolution of PAM is determined by the time-resolved acoustic detection. Depending on the method used to determine the lateral resolution, there are two types of PAM: acoustic-resolution PAM (AR-PAM)[62] and optical-resolution PAM (OR-PAM)[38].

\subsubsection{Acoustic-Resolution PAM (AR-PAM)}

In AR-PAM, a focused ultrasonic transducer is used to detect the acoustic signal generated within its focal zone under diffusive illumination of a pulsed laser source.[63] AR-PAM is more desirable for deep-tissue imaging because the acoustic scattering is relatively small compared with the optical scattering in biological tissues and a focused ultrasonic transducer can suppress signals generated outside its focal zone. [46] A dark-field AR-PAM system was developed and demonstrated to achieve a $45 \mu \mathrm{m}$ lateral resolution and $3 \mathrm{~mm}$ imaging depth at $50 \mathrm{MHz}$ ultrasonic frequency.[62] With a transducer of lower frequency as $5 \mathrm{MHz}$, AR-PAM has been proved to be able to image as deep as 38mm.[64] The lateral resolution of AR-PAM is determined by the ultrasonic focal spot and the axial resolution is determined by the ultrasonic center frequency and bandwidth.[65]

\subsubsection{Optical-Resolution PAM (OR-PAM)}

To improve the resolution of AR-PAM, an ultrasonic transducer with higher frequency can be used. However, the severely attenuated acoustic signal limits the imaging depth even lower than the optical penetration. [66] Different from AR-PAM, optical focusing is used in OR-PAM to provide better lateral resolution for PAM imaging and both focused transducer and unfocused transducer can be used. OR-PAM can provide lateral resolution ranging from a few hundred nanometers to a few micrometers determined by the optical focal spot. Same as many other optical imaging techniques, the image depth is limited by optical scattering in biological tissue within the 
ballistic regime, but OR-PAM still benefits from its absorption-specific imaging contrast.[67] Thus, OR-PAM is promising to image low scattering tissues such as eye.[39, 63]

\subsubsection{Wave Equation and PAM Fundamentals}

In response to a heat source $H(\boldsymbol{r}, t)$, without considering thermal diffusion and kinematical viscosity, the pressure $p(\boldsymbol{r}, t)$ at position $\boldsymbol{r}$ and time $t$ in an acoustically homogeneous liquid-like medium obeys the following wave equation[68]:

$$
\nabla^{2} p(\boldsymbol{r}, t)-\frac{1}{c^{2}} \frac{\partial^{2}}{\partial t^{2}} p(\boldsymbol{r}, t)=-\frac{\beta}{C_{p}} \frac{\partial}{\partial t} H(\boldsymbol{r}, t)
$$

where $p(\boldsymbol{r}, t)$ is the wave pressure at position $\boldsymbol{r}$ and time $t, c$ is the speed of sound, $\beta$ is the isobaric volume expansion coefficient, $C_{p}$ is the heat capacity and $H(\boldsymbol{r}, t)$ is the heating function defined as the thermal energy deposited by the electromagnetic radiation per unit time and per unit volume.

We can rewrite Equation 1.11 in terms of a velocity potential $\phi$ as:

$$
\left(\nabla^{2}-\frac{1}{c^{2}} \frac{\partial^{2}}{\partial t^{2}}\right) \phi(\boldsymbol{r}, t)=-\frac{\beta}{\rho_{f} C_{p}} H(\boldsymbol{r}, t),
$$

where $p=-\rho_{f} \partial \phi / \partial t, \rho_{f}$ is the fluid density.

Based on Green's function, the solution to the wave equation for the velocity potential can be expressed as:

$$
\phi(\boldsymbol{r}, t)=\frac{\beta}{4 \pi \rho_{f} C_{p}} \int_{0}^{t} d t^{\prime} \int d \boldsymbol{r}^{\prime} g\left(\boldsymbol{r}, t \mid \boldsymbol{r}^{\prime}, t^{\prime}\right) H\left(\boldsymbol{r}^{\prime}, t^{\prime}\right)
$$

where $\mathrm{g}\left(\boldsymbol{r}, t \mid \boldsymbol{r}^{\prime}, t^{\prime}\right)$ is the Green's function for an infinite medium.

The heating function can be written as the product of a spatial absorption function of objects $A(\boldsymbol{r})$ and a temporal illumination function for stimulating $I(t)$ : 


$$
H(\boldsymbol{r}, t)=A(\boldsymbol{r}) I(t),
$$

Particularly, if $I(t)=\delta(t)$, the initial photoacoustic pressure at position $\boldsymbol{r}$ equals

$$
p_{0}(\boldsymbol{r})=\Gamma(\boldsymbol{r}) A(\boldsymbol{r}),
$$

where $\Gamma(\tau)$ is the Grüneisen parameter equal to $c^{2} \beta / C_{p}$.

The axial resolution of PAM is determined by the center frequency and bandwidth of the ultrasonic transducer, which can be expressed as:

$$
R_{\text {axial }}=\frac{c}{2 \cdot f_{c} \cdot B W},
$$

where $\mathrm{c}$ is the speed of sound, $f_{c}$ is the center frequency of the ultrasonic transducer and $B W$ is the bandwidth. For example, in our original PAM system an ultrasonic transducer with center frequency of $30 \mathrm{MHz}$ and $-6 \mathrm{~dB}$ bandwidth of $53 \%$ was used. The axial resolution of this transducer was $46 \mu \mathrm{m}$. As the Equation 1.16 shows, the ultrasonic transducer with higher center frequency will produce better axial resolution. However, considering the propagation of photoacoustic signal in tissues, the acoustic attenuation coefficient $\alpha_{f}$ is defined as:

$$
\alpha_{f}=\alpha_{0} f^{n},
$$

where $\alpha_{0}$ is a constant equal to the attenuation coefficient at $1 \mathrm{MHz}, f$ is the acoustic frequency and $\mathrm{n}$ is a frequency-dependent parameter. For many biological tissues, $n \approx 1$ and $\alpha_{0} \approx 0.5 \mathrm{~dB}$. $M \mathrm{~Hz}^{-1} \cdot \mathrm{cm}^{-1}$.[69] Therefore, higher frequency components of photoacoustic signal will attenuate more than lower frequency components.

\subsection{Combination of OCT and PAM}

Multimodal imaging with combined OCT and PAM is able to provide both the optical scattering and optical absorption contrasts of a sample. Previous studies about integrated OCT and PAM imaging have demonstrated the complementary nature of the contrasts provided by the two 
imaging technologies.[67, 70] The combined OCT and PAM is thus potentially able to provide more comprehensive imaging of a subject. As an example, combined OCT and PAM is able to provide simultaneous imaging of the retinal structures, retinal vasculature, and melanin in the retinal pigment epithelium.[38, 71] By combining Doppler OCT and multi-spectral PAM it is also possible to quantify the metabolism of Oxygen in biological tissues in vivo. [72, 73]

These two technologies, however, have different requirements for their light sources. OCT needs a broadband light source to achieve depth resolution. PAM requires a pulsed laser to deposit the light energy in the absorber in a time scale shorter than the requirements of thermal and stress confinement.[74] Thus, two different light sources are usually used to achieve simultaneous OCT and PAM imaging.[67]

\subsection{Optical Coherence Photoacoustic Microscopy (OC-PAM)}

Using a single light source to achieve simultaneous OCT and PAM imaging was first reported in 2012, in which a dye-laser-based pulsed broadband light source centered at $580 \mathrm{~nm}$ was used.[75] The technique was termed optical coherence photoacoustic microscopy (OC-PAM) because OCT and PAM are an integral part of the technology. To demonstrate the feasibility of imaging biological tissues the system was successfully used to image a mouse ear in a transmission mode. Lee et al. described an OC-PAM system in the near infrared (NIR) spectral range.[76] However, no in vivo imaging of biological samples was presented in their report.

Because of its better penetration depth and better tolerance of the subject, NIR light is more suitable for imaging the retina. Most ophthalmic OCT systems are in the NIR and have a center wavelength of $\sim 830 \mathrm{~nm} .[1,3,35]$ As a result, we believe an OC-PAM system at a center wavelength of around $830 \mathrm{~nm}$ will be more suitable for retinal imaging. Since the optical absorption coefficient of hemoglobin in the NIR is much smaller than that within the visible spectrum, a PAM 
in NIR would not be expected to provide good contrast for imaging the retinal vasculature. In contrast to hemoglobin, melanin in the retinal pigment epithelium (RPE) cells and in the choroid has broad absorption spectrum extending from the visible to the NIR.[77, 78] As a result, we expect that melanin will be the major contributor to the signals of the NIR-PAM imaging.

\subsection{Significance and Aims of the Study}

\subsubsection{Significance}

The RPE is a monolayer of pigmented cells between the photoreceptors and choriocapillaris in the eye. There are two major pigments in RPE, melanin and lipofuscin. RPE melanin has a photoprotective role by absorbing radiation and scavenging free radicals and reactive oxygen species (ROS).[79, 80] On the other hand, lipofuscin is a by-product of phagocytosis of the

photoreceptor outer segments, which accumulates with aging of the retina. Excessive levels of lipofuscin accumulation could compromise essential RPE functions and contribute to the pathogenesis of age-related macular degeneration (AMD)[81], a leading cause of blinding diseases in developed countries. Hence, in vivo imaging of melanin and lipofuscin can provide important aging information of the retina, which is important for AMD research and clinical diagnosis.[82]

In the NIR spectrum range, PAM is able to provide melanin-specific imaging of the retina and OCT is able to image scattering contrast, which is related to the amount of lipofuscin accumulated in the RPE layer. Thus, the proposed OC-PAM system is potentially able to provide a unique technique to image melanin and lipofuscin specifically, which will benefit the diagnosis and research of AMD. 


\subsubsection{Aims of the Study}

Aim 1: To develop optical coherence photoacoustic microscopy (OC-PAM) for in vivo multimodal retinal imaging with a pulsed broadband NIR light source.

Since the OC-PAM system with pulsed visible light source has been developed and its feasibility of imaging biological tissues was demonstrated, to provide melanin-specific imaging of the retina, a pulsed NIR light source would be used instead in the proposed OC-PAM system.

Aim 2: To develop OC-PAM for in vivo multimodal imaging with an intensity-modulated continuous-wave light source in the NIR.

Previous OC-PAM systems used a pulsed broadband light source in which each light pulse generates one depth scan (A-line) for both OCT and PAM. In this configuration the OCT image quality highly depends on the pulse-to-pulse stability of the light source without averaging. In addition, laser safety is always a concern for in vivo applications, especially for eye imaging with a pulsed light source. Therefore, a continuous wave (CW) light source is desired for OC-PAM applications.

Aim 3: In vivo application of NIR OC-PAM on small animals.

To demonstrate the feasibility of the NIR OC-PAM system in imaging living animals would provide evidence for further applications in clinical and disease diagnosis. Therefore, the system will be used for in vivo imaging of rats or mice.

Aim 4: To conduct a phantom study and extract quantitative information of the optical properties from OC-PAM.

The quantitative information of the optical properties extracted from the proposed NIR OCPAM system is potentially able to provide a unique technique to evaluate the existence of melanin and lipofuscin specifically. Thus, to conduct the phantom study and convert the relationship 
between image intensity of OCT and PAM to the relationship between the optical scattering property and optical absorption property would be strong evidence for practical application of the proposed NIR OC-PAM system. 


\section{CHAPTER 2. EFFECT OF CONTACT LENS ON OPTICAL COHERENCE TOMOGRAPHY IMAGING OF RODENT RETINA[83]}

\subsection{Introduction}

Since the development of spectral-domain (SD) detection technique, OCT is rapidly becoming an indispensable non-invasive imaging modality for the diagnosis and treatment monitoring of various ocular diseases in human.[33, 34] SD-OCT has also been widely used to image the eye of animals including small animal models of various retinal diseases.[84-90] By providing unprecedented in vivo high resolution visualization of the retinal structures small animal ophthalmic OCT has enabled the study of the time course of retinal diseases using the same animal. We anticipate that with the development of automatic segmentation software and more user friendly interface OCT will play a more and more important role in ophthalmic research. However, the diagnostic capability of OCT in both clinical applications and research highly depends on its image quality.

In addition to the dependence on the technology of an OCT machine, the imaging quality of retina, e.g. lateral resolution and signal-to-noise ratio (SNR), also depends on the optical properties of the anterior segment of the eye including its optical aberrations and transparency. Optical aberrations have been recognized as the ultimate limiting factor for the achievable lateral resolution of retinal images. To improve the lateral resolution of retinal imaging many groups are working on adaptive optics to correct the optical aberrations of the eye.[91-94] Studies have shown that rodent eyes have high amplitude of various orders of optical aberrations.[95-98] Application of adaptive optics has successfully improved the lateral resolutions of rodent retinal imaging.[94]

There is an additional hurdle in achieving high quality imaging of rodent retina: cataract formation when the animal is anesthetized. To keep the eye transparent during imaging, 
examination time is required to be short, and artificial tears need to be applied to the cornea repeatedly.[84] However, even with the application of artificial tears in our experiments cataract can still occur when the imaging time lasts longer (e.g. more than 10 minutes). To solve this problem powerless contact lenses have been used in imaging rodent retina with various imaging modalities.[99, 100]

Contact lenses may have additional benefits for imaging the rodent retina. Gesine et al. used a custom-made contact lens (focal length: $10 \mathrm{~mm}$ ) to reduce the risk of corneal dehydration and edema and to act as a collimator.[101] In a previous human study, prescribed contact lens was used to partially compensate for corneal aberrations of human eye for adaptive optics scanning laser ophthalmoscopy.[92, 102] Srinivasan et al. found that imaging small animal eye with a 'contact lens’ made from a flat microscope coverslip and Hydroxypropyl Methylcellulose can effectively remove the refractive power of the air-corneal interface and the aberrations from irregularities in the cornea while maintaining corneal hydration and clarity during imaging.[98] However, since this type of 'contact lens' is used to cancel the refractive power of the eye it is incompatible with standard retinal imaging techniques.

We used a powerless contact lens on rat's eye to slow cataract formation in OCT imaging experiments. We found that the retinal OCT images had better quality after applying a contact lens. The purpose of this study is to verify our observations by directly compare the OCT images of rat retina acquired with and without a contact lens.

\subsection{Methods}

\subsubsection{Experimental System}

A schematic of the experimental system is shown in Figure 2.1. A three-module superluminescence diode (SLD, T-840 Broadlighter, Superlum Diodes Ltd. Moscow, Russia) with 
a center wavelength of $840 \mathrm{~nm}$ and full-width-half-magnitude(FWHM) bandwidth of $100 \mathrm{~nm}$ was used in the system, which can achieve a depth resolution of $\sim 3 \mu \mathrm{m}$ in tissue. In the sample arm, the light was collimated, scanned by an $\mathrm{X}-\mathrm{Y}$ galvanometer scanner, and then focused by an achromatic lens (L1, $\mathrm{f}=75 \mathrm{~mm})$. For imaging the retina in vivo, an ocular lens (L2, $\mathrm{f}=19 \mathrm{~mm})$ was added. The light beam was collimated after the ocular lens and focused onto the retina by the anterior segment of the eye. In the detection arm, the reflected light from the sample and reference arms was collimated and detected by a spectrometer, which consists of an 1200 line/mm transmission grating, a multi-element imaging lens ( $\mathrm{f}=150 \mathrm{~mm})$, and a line scan CCD camera (AVIIVA EM4 2k 4×12bits, 2048 pixels with 14 micron pixel size, e2V). An image acquisition board (NI PCI-1429) was used to acquire the image captured by the CCD camera and transfer the data to a computer for signal processing and image display.

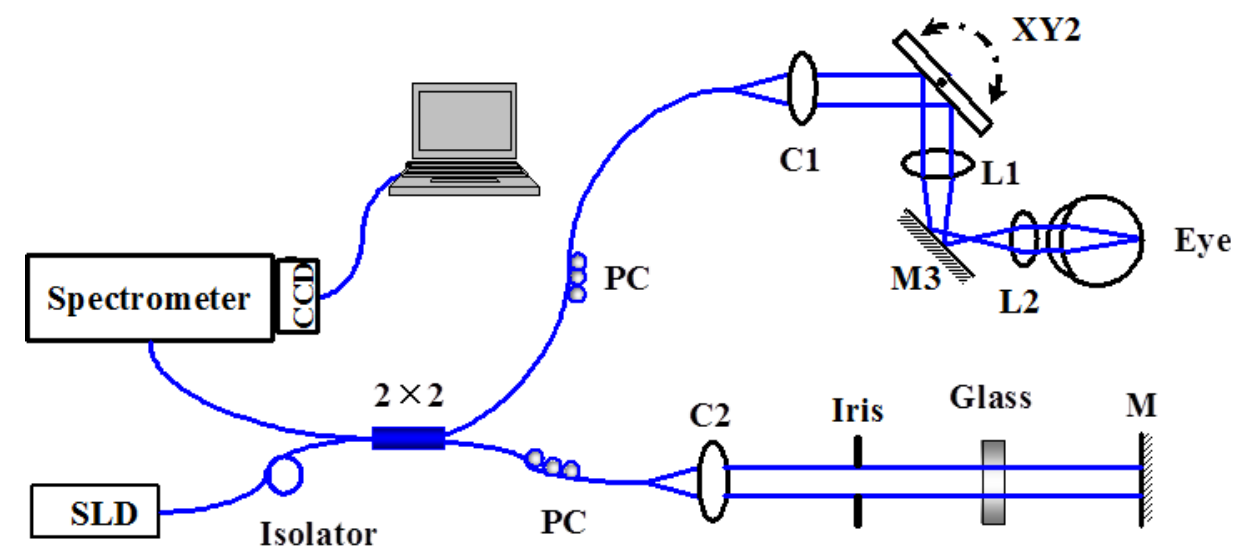

Figure 2.1 Schematic of the experimental OCT system. M: Mirror; C1, C2: Collimator; L1, L2: Lens; PC: Polarization controller.

\subsubsection{Animal Imaging}

To evaluate the effect of contact lens on OCT retinal imaging, the system was applied to imaging the normal rat retina in vivo. The animal (Sprague Dawley rat, body weight: $450 \mathrm{~g}$, Charles 
Rivers) was anesthetized by intraperitoneal injection of a cocktail containing ketamine (54 mg/kg body weight) and xylazine (6 mg/kg body weight). The pupil was dilated with $10 \%$ phenylephrine solution. All experiments were performed in compliance with the guidelines of the University of Southern California's Institutional Animal Care and Use Committee. When no contact lens was used, drops of artificial tears were applied to the eyes every 2 minutes to prevent dehydration of the cornea and cataract formation. After anesthetization, the animals were restrained in a mounting tube, which was fixed on a five-axis platform. The light power in front of the eye was about 900 $\mu \mathrm{W}$, which is below the ANSI safety limits for eye imaging.[103] The rat eye was first imaged without contact lens. The image quality on the real-time display was optimized by adjusting the ocular lens. Immediately after the imaging, we put a few drops of artificial tears onto a powerless contact lens (Cantor + Nissel, PMMA 2.70/5.20, radius of curvature of the central optic zone: 2.70mm; diameter: 5.20mm) and put it on the imaged eye gently. During this procedure, we tried our best to keep the eye in the same position. We then acquired another set of retinal OCT images. With contact lens we put no artificial tears on the eye during the imaging procedure. After all the images were acquired, we post-processed them for analyses.

\subsection{Results}

\subsubsection{Image Overview}

Figure 2.2(a-d) shows two pairs of en-face images (OCT fundus image) of the acquired threedimensional dataset for the retina of two rats with and without contact lens, respectively. The images cover an area of $1.5 \times 1.5 \mathrm{~mm}^{2}$, consisting of 512 (horizontal) $\times 128$ (vertical) pixels, and are displayed in grayscale. According to the retinal blood vessel pattern, we can see that the position of the eye is almost the same before and after putting on the contact lens. Compared with the fundus image without contact lens, the one with contact lens obviously provides higher resolution (sharper 
blood vessel image) and shows more small blood vessels. The contrast and sharpness of blood vessels increase significantly after applying contact lens.
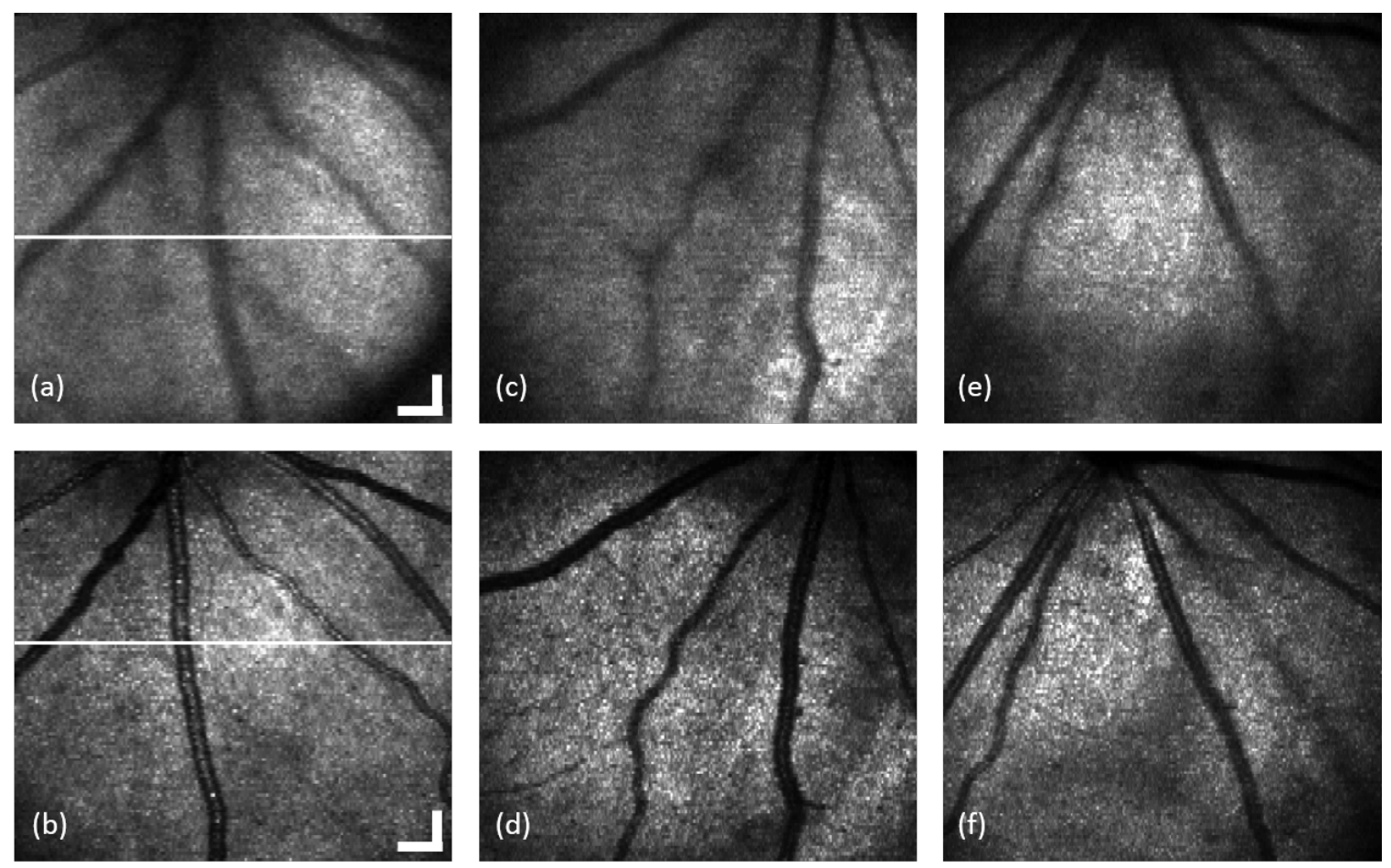

Figure 2.2 OCT fundus images generated from the acquired 3D OCT datasets for the retina of rat 1 (a and b), rat 2 (c and d) and rat 3 (e and f), with (b, d, f) and without (a, c, e) contact lens, respectively. (Bar: $150 \mu \mathrm{m}$ )

Figure 2.3 shows two pairs of B-scan images for the retina of two rats with and without contact lens, respectively. The images consist of $2048 \mathrm{~A}$-lines and are displayed in grayscale with the same dynamic range. From the brightness of these images we can see that the signal intensity of the Bscan images with contact lens is higher than that without contact lens. The retinal layers in the OCT images also become clearer after applying contact lens. 

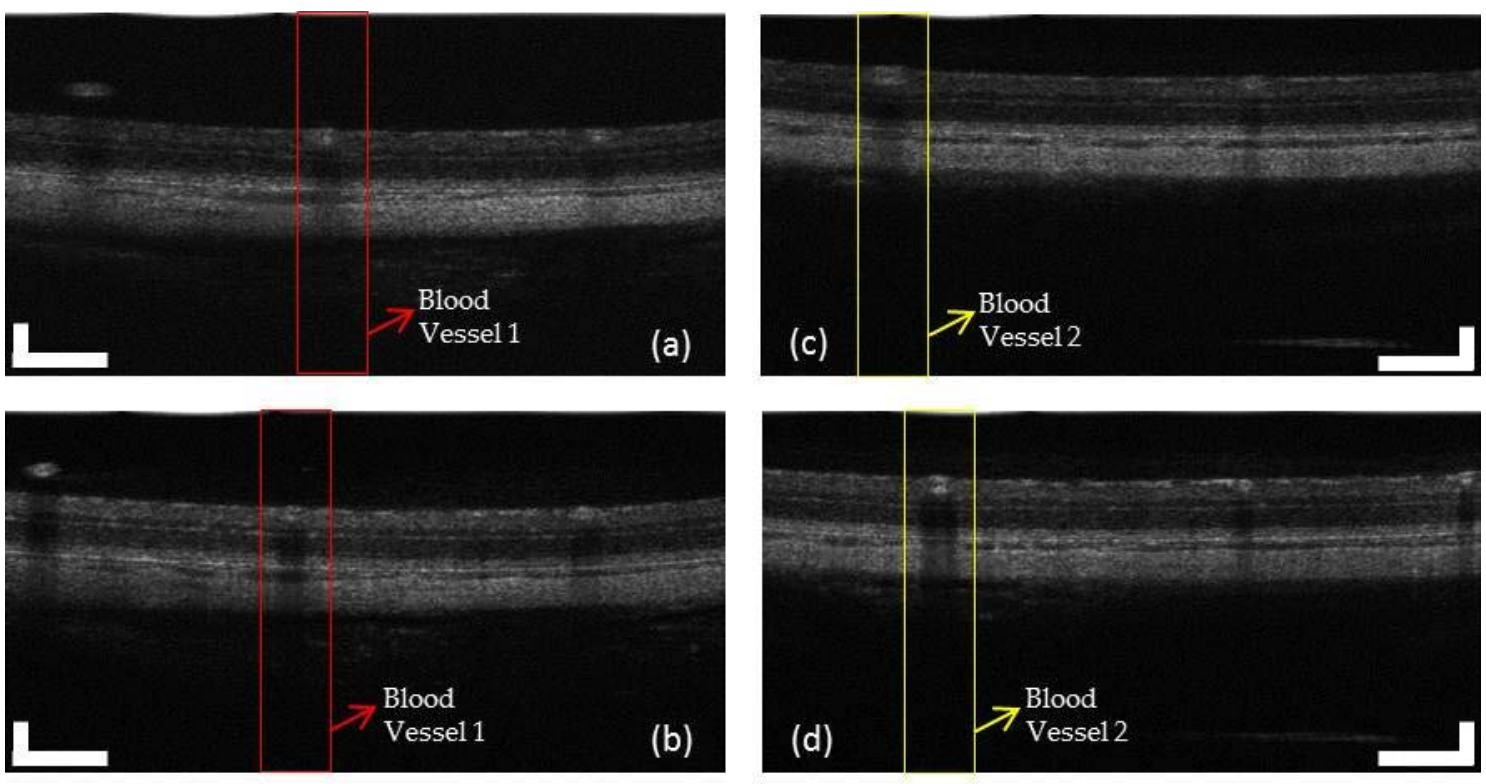

Figure 2.3 Two pairs of OCT images for the retina of two rats with and without contact lens. (a) and (b): OCT images of rat 1 without and with contact lens. (c) and (d): OCT images of rat 2 without and with contact lens. The images consist of 2048 A-lines. (Bar:

$200 \mu \mathrm{m})$

\subsubsection{Spatial Resolution}

We evaluate the improvement of lateral resolutions of the OCT images by comparing the sharpness of the retinal blood vessels on the corresponding OCT fundus images. To compare the sharpness of retinal blood vessels, we manually identified the OCT cross-sectional images with and without contact lens that contain the same blood vessels at the same location. Figure 2.3 shows two pairs of the OCT images for two rats (a and c: rat 1 and 2 without contact lens; b and d: rat 1 and 2 with contact lens). We then calculated the vessel profile by summing the pixel intensities in the depth direction, which are shown in Figure 2.4. As shown in Figure 2.4, the vessel profiles in the images with contact lens are sharper than that without contact lens for both rats. The central peak (higher reflection) in the vessel profiles is caused by the specular reflection of the vessel wall in the central region of the vessels. The retinal blood vessel profiles are typical when extracted from fundus images acquired by imaging technologies like fundus camera, SLO or OCT.[104, 105] 


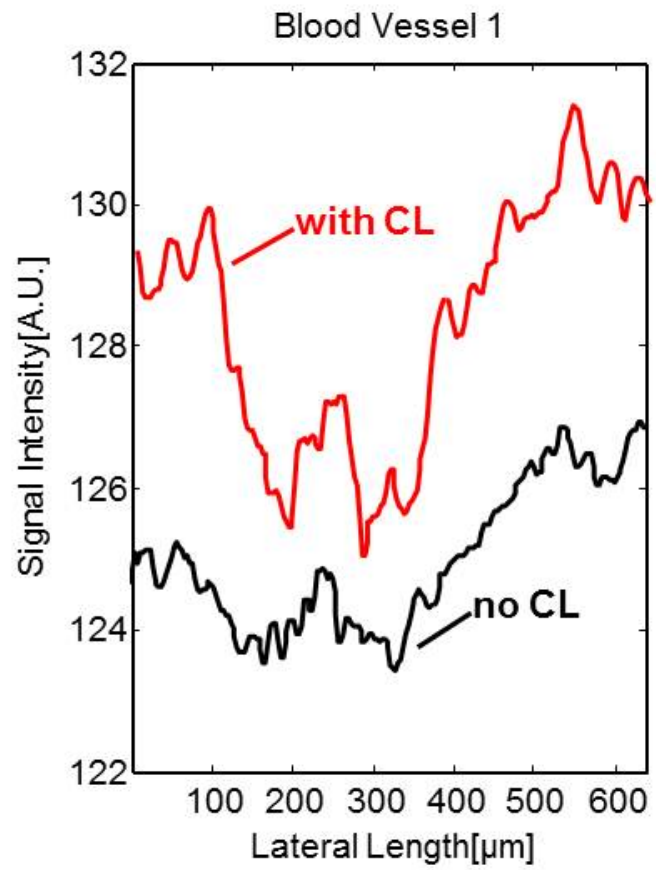

(a)

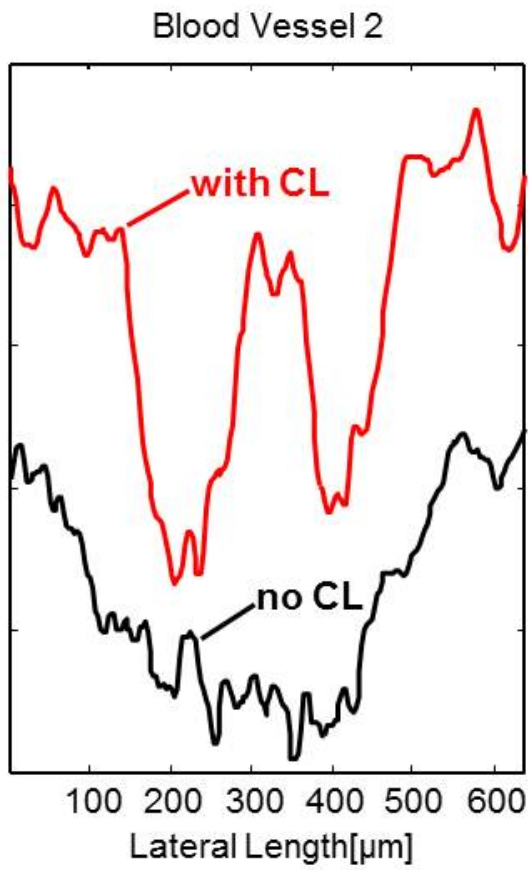

(b)

Figure 2.4 Blood vessel profiles generated by summation of the OCT signal along the depth direction. (a) The profile of blood vessel 1 in the area marked red in Figure 2.3 (a-b); (b) The profile of blood vessel 2 in the area marked yellow in Figure 2.3(c-d).

\subsubsection{Signal to Noise Ratio}

We compared the signal-to-noise ratio (SNR) between the OCT images with and without contact lens. We manually chose several pairs of B-scan images at the same location of the retina in the corresponding dataset. Figure 2.5(a)-(b) show one pair of corresponding B-scans chosen from the location marked as a white line in Figure 2.2(a)-(b), with and without contact lens, respectively. A-line signals (or OCT signal) strength at locations marked as white lines on the Bscan images are shown in Figure 2.5(c)-(d). The A-line signals are displayed in linear scale. We can see that at the same location the OCT signal is much stronger with contact lens than that without contact lens. 


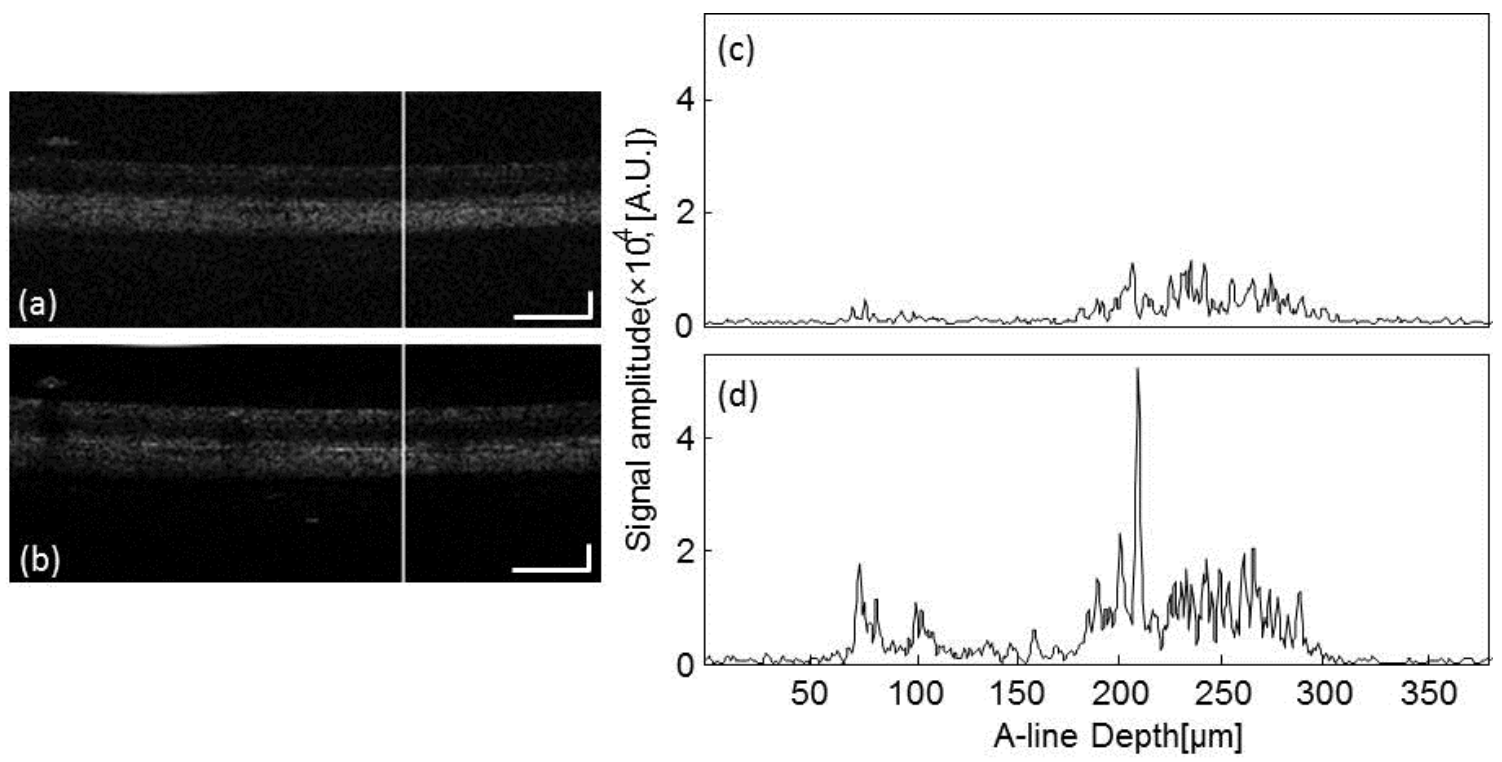

Figure 2.5 OCT signals at the same location of the retina chosen from a pair of OCT images with and without contact lens, the locations of which are marked as white lines in Figure 2(a) and Figure 2(b). (c) OCT signal without contact lens at the location marked in (b). (Bar: $200 \mu \mathrm{m}$ )

Table 2.1 SNR Calculation of OCT A-lines with and without contact lens

\begin{tabular}{|c|c|c|c|c|c|c|c|c|}
\hline & & \multicolumn{3}{|c|}{ No lens } & \multicolumn{3}{|c|}{ With lens } & \multirow[b]{2}{*}{$\begin{array}{c}\Delta \text { SNR } \\
\text { (dB) }\end{array}$} \\
\hline & & $\begin{array}{c}\text { signal } \\
\text { strength } \\
\text { (counts) }\end{array}$ & $\begin{array}{c}\text { noise } \\
\text { strength } \\
\text { (counts) }\end{array}$ & $\begin{array}{l}\text { SNR } \\
(\mathrm{dB})\end{array}$ & $\begin{array}{c}\text { signal } \\
\text { strength } \\
\text { (counts) }\end{array}$ & $\begin{array}{c}\text { noise } \\
\text { strength } \\
\text { (counts) }\end{array}$ & $\begin{array}{l}\text { SNR } \\
(\mathrm{dB})\end{array}$ & \\
\hline \multirow{3}{*}{$\begin{array}{c}\text { Fundus } \\
1\end{array}$} & A-line \#1 & $2.11 \times 10^{3}$ & 531.93 & 11.98 & $4.84 \times 10^{3}$ & 509.92 & 19.54 & 7.56 \\
\hline & A-line \#2 & $2.03 \times 10^{3}$ & 585.14 & 10.79 & $5.96 \times 10^{3}$ & 597.09 & 19.98 & 9.19 \\
\hline & A-line \#3 & $2.71 \times 10^{3}$ & 548.79 & 13.87 & $6.13 \times 10^{3}$ & 601.64 & 20.16 & 6.29 \\
\hline \multirow{3}{*}{$\begin{array}{c}\text { Fundus } \\
2\end{array}$} & A-line \#4 & $3.13 \times 10^{3}$ & 555.58 & 15.01 & $5.91 \times 10^{3}$ & 561.86 & 20.44 & 5.43 \\
\hline & A-line \#5 & $3.12 \times 10^{3}$ & 531.73 & 15.37 & $4.43 \times 10^{3}$ & 540.53 & 18.27 & 2.90 \\
\hline & A-line \#6 & $3.90 \times 10^{3}$ & 564.10 & 16.80 & $7.08 \times 10^{3}$ & 611.34 & 21.27 & 4.47 \\
\hline \multirow{3}{*}{$\begin{array}{c}\text { Fundus } \\
3\end{array}$} & A-line \#7 & $3.05 \times 10^{3}$ & 558.51 & 14.74 & $7.85 \times 10^{3}$ & 600.12 & 22.33 & 7.59 \\
\hline & A-line \#8 & $2.47 \times 10^{3}$ & 570.37 & 12.73 & $7.51 \times 10^{3}$ & 544.96 & 22.78 & 10.06 \\
\hline & A-line \#9 & $3.26 \times 10^{3}$ & 609.55 & 14.57 & $6.54 \times 10^{3}$ & 612.62 & 20.57 & 5.99 \\
\hline
\end{tabular}


We calculated the SNR of nine pairs of A-lines from three pairs of OCT images of three different rat eyes. The results are shown in Table 2.1. The SNR is calculated by using the formula

$S N R=20 \log _{10} \frac{S_{\text {signal }}}{N_{\text {noise }}}$. In the calculation we determined the noise level by averaging the OCT signals before the top surface of the retina and that below the choroid, while the signal is determined by averaging the OCT signals between the RNFL and the choroid. From the results we can see that the SNR increased $2.9 \mathrm{~dB}$ to $10 \mathrm{~dB}$ after applying contact lens on the rat eyes.

\subsection{Discussion}

From the experimental results we can see that both the spatial resolution and SNR of the OCT images were improved significantly after applying contact lens on the rat eyes. The results suggest that after applying contact lens the probing beam was focused better on the retina (smaller focal spot). Since the contact lens used in our experiments is powerless and each OCT image was acquired by adjusting the ocular lens to achieve the best image quality, we believe that the improvement of image quality is not caused by focus change.

We hypothesize that the image quality was improved as a result of smaller wave-front error of the anterior segment of the eye with contact lens. We all know that the corneal surface of rodent eyes frequently has various defects. These defects induce wave-front errors, which will add to the aberrations of the rodent eye. Since we added artificial tears in the contact lens before put it on the eye, the defects of the corneal surface may be smoothed out by the post-lens tear film. As a result, the aberrations of the eye may be partially compensated by the contact lens and lead to better OCT image quality. Since we do not have a wave-front sensor to measure the aberrations of the rodent eyes the hypothesis was not verified in our lab. Further experiments using a wave-front sensor may help verify the hypothesis. 

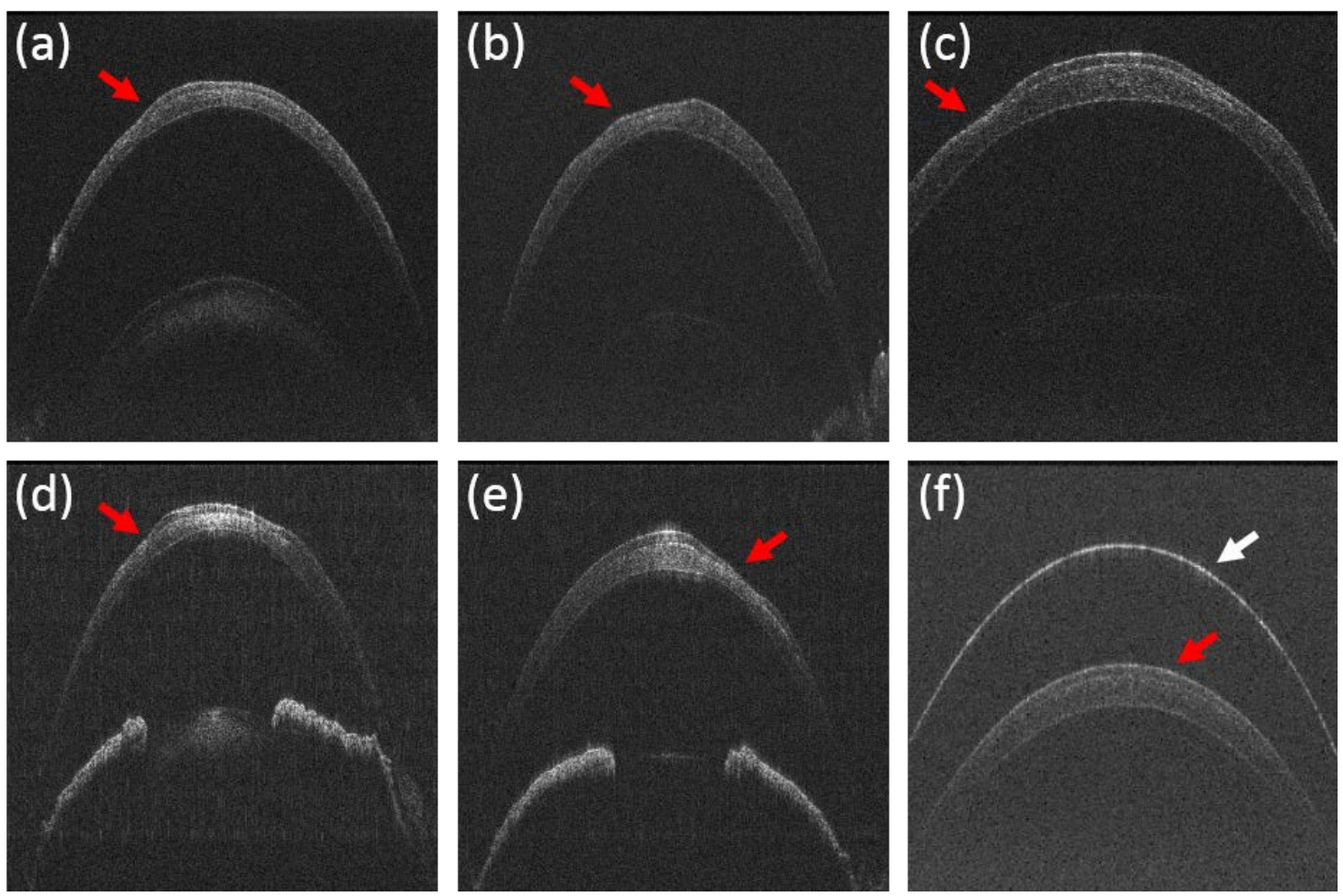

Figure 2.6 Typical OCT images of rat's cornea. (a)-(c) Cornea images of Sprague Dawley rats in different ages, the red arrows indicate the location of cornea defects. (d) and (e) Cornea images of Long Evans rats in different ages, the red arrows indicate the location of cornea defects. (f) The rat's cornea with contact lens, the white arrow indicates the front surface of contact lens and red arrow shows the interface between rear surface of contact lens and rat's cornea. The images consist of 2048 A-lines.

As a follow-up study of this topic, another OCT system was built to image the anterior segment of rat's eye in order to observe the condition of cornea. Totally 26 rats in different ages and different strains were imaged, among which 16 were Sprague Dawley rats and 10 were Long Evans rats. Different levels of defects were found on almost every rat's cornea. Figure 2.6 shows some of the typical cornea images. Figure 2.6(a)-(c) show cornea images of Sprague Dawley rats in different ages and the red arrows indicate the location of cornea defects. Figure 2.6(d) and (e) show cornea images of Long Evans rats in different ages and the red arrows indicate the location of cornea defects. The defects found on rat cornea are most likely some raised or sunken areas on the surface of cornea. Figure 2.6(f) shows the rat's cornea with contact lens, the white arrow indicates the front 
surface of contact lens and red arrow shows the interface between rear surface of contact lens and rat's cornea. Table 2.2 summarizes the strains, ages and body weight of the rats shown in Figure 2.6.

Table 2.2 Summarized information of rats shown in Figure 2.6

\begin{tabular}{ccccccc}
\hline Figure No. & (a) & (b) & (c) & (d) & (e) & (f) \\
\hline \multirow{2}{*}{ Rat strain } & Sprague & Sprague & Sprague & Long & Long & Sprague \\
Dawe & Dawley & Dawley & Dawley & Evans & Evans & Dawley \\
B weeks & 3 months & 9 months & 4 weeks & 6 weeks & 9 months \\
Body weight & $330 \mathrm{~g}$ & $385 \mathrm{~g}$ & $652 \mathrm{~g}$ & $233 \mathrm{~g}$ & $300 \mathrm{~g}$ & $626 \mathrm{~g}$ \\
\hline
\end{tabular}

The results in this study show the structure of anterior segment of rat eye, and cornea defects were observed on most of the rats' eye. These uneven surfaces of cornea may induce severe aberrations to the eye when performing optical imaging such as OCT on the eye. After applying contact lens, the surface of eye became smooth and even, which improves the optics system of anterior segment of rat eye. This supplement study is a support of our previous hypothesis that the improvement of OCT image quality by applying contact lens is a result of compensation of the optical aberration caused by the defects of rat cornea.

\subsection{Conclusion}

In conclusion, we have for the first time investigated the effect of powerless contact lens on OCT imaging of rodent retina. By comparing the OCT images of rat retina with and without contact lens, we have demonstrated that using contact lens on rat eyes can significantly improve the lateral resolution and SNR of OCT images. This work demonstrated that contact lens can not only help prevent cataract formation in rodent eyes during imaging but also significantly improve the image quality of rodent retina. 


\section{CHAPTER 3. OPTICAL COHERENCE PHOTOACOUSTIC MICROSCOPY FOR IN VIVO MULTIMODAL RETINAL IMAGING[106]}

\subsection{Introduction}

Optical coherence tomography (OCT)[1, 2, 34, 84, 98] is a low-coherence interferometrybased three-dimensional imaging modality, which has been widely used in ophthalmology and animal research for high-resolution imaging of the retina. In OCT, the contrasts are provided by the photons backscattered from the biological tissues, which carry information about mainly their scattering properties. OCT can thus provide imaging of the micro-structures of biological tissues like the layered structures of the retina. The development of OCT has greatly changed the landscape of diagnosis and patient care in ophthalmology clinics.

Photoacoustic microscopy (PAM)[37-41] is a novel microscopic three-dimensional noninvasive imaging modality that is used for imaging the microvasculature and the associated blood oxygenation of biological tissues.[42] PAM is based on the optical-absorption properties of biological tissues. When irradiated by a short laser pulse, the optical energy absorbed by a substance, such as hemoglobin or melanin in tissue, is converted to heat, which induces localized thermo-elastic expansion and leads to the generation of wideband ultrasonic waves. The ultrasonic waves can be detected with an ultrasonic transducer to reconstruct an image mapping the location and absorption strength of the absorbers.

Multimodal imaging with combined OCT and PAM is able to provide both the optical scattering and optical absorption contrasts of a sample. Previous studies about integrated OCT and PAM imaging have demonstrated the complementary nature of the contrasts provided by the two imaging technologies. The combined OCT and PAM is thus potentially able to provide more comprehensive information of a subject.[67, 70] As an example, combined OCT and PAM is able 
to provide simultaneous imaging of the retinal structures, retinal vasculature, and melanin in the retinal pigment epithelium.[39, 71] By combining Doppler OCT and multi-spectral PAM it is also possible to quantify the metabolism of Oxygen in biological tissues in vivo.[72, 73]

The two technologies, however, have different requirements for their light sources. OCT needs a broadband light source to achieve depth resolution whereas PAM requires a pulsed laser to deposit the light energy in the absorber in a time scale shorter than the requirements of thermal and stress confinement.[74] Thus, two different light sources are usually used to achieve simultaneous OCT and PAM imaging.

Using a single light source to achieve simultaneous OCT and PAM imaging was first reported in 2012, in which a dye-laser-based pulsed broadband light source centered at $580 \mathrm{~nm}$ was used.[75] The technique was termed optical coherence photoacoustic microscopy (OC-PAM) because OCT and PAM are an integral part of the technology. To demonstrate the feasibility of imaging biological tissues the system was successfully used to image mouse ear in a transmission mode. Lee et al. described an OC-PAM system in the near infrared (NIR) spectral range.[76] However, no in vivo imaging of biological samples was presented in their report.

Because of its better penetration depth and better tolerance by the eye of an imaging subject, NIR light is more suitable for imaging the retina. Most ophthalmic OCT systems are using NIR light source, which has a center wavelength of $\sim 830 \mathrm{~nm}$. $[1,3,35]$ As a result, we believe an OCPAM system with a light source at a center wavelength of around $830 \mathrm{~nm}$ will be more suitable for retinal imaging. Since the optical absorption coefficient of hemoglobin in the NIR spectrum range is much smaller than that within the visible spectrum, a PAM in NIR would not be expected to provide good contrast for imaging the retinal vasculature. In contrast to hemoglobin, melanin in the RPE cells and in the choroid has broad absorption spectrum extending from the visible to the 
NIR.[77, 78] As a result, we expect that melanin will be the major contributor to the signals of the NIR-PAM imaging mode.

\subsection{Methods}

\subsubsection{Experimental System}

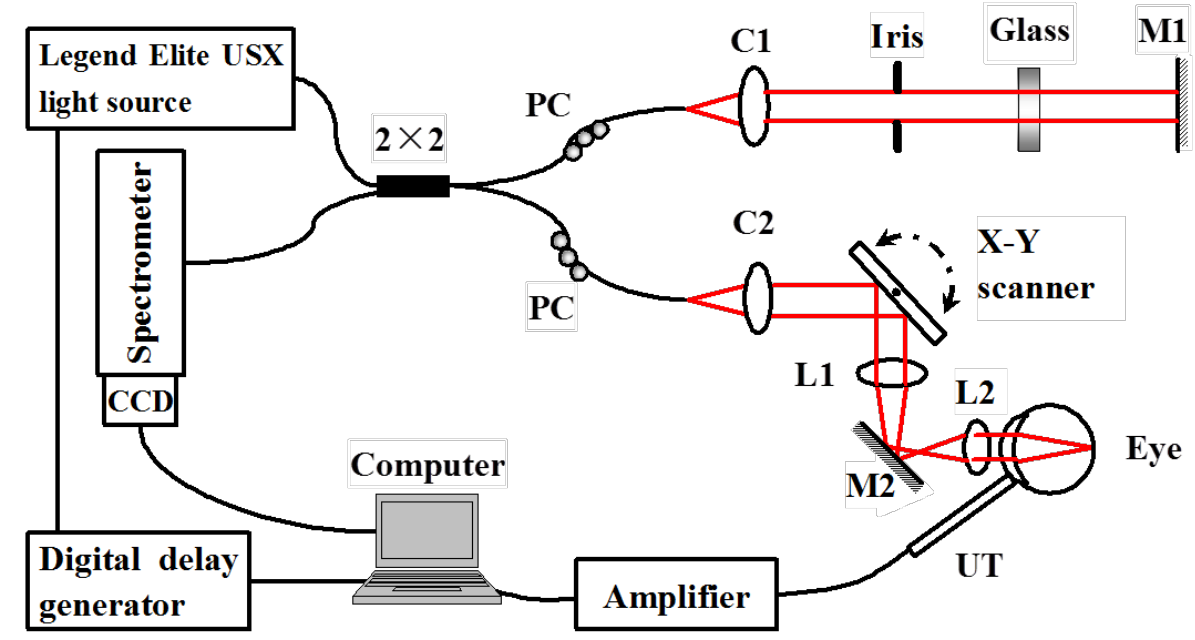

Figure 3.1 Schematic of the experimental system of a fiber-based OC-PAM. L1, L2: lens; PC: polarization controller; UT: ultrasonic transducer; M1, M2: mirror; C1, C2: collimator.

Figure 3.1 shows a schematic of the experimental system. A commercial ultrafast Ti: sapphire laser amplifier (Legend Elite HE+ USX-10K-I, Coherent Inc.) operating under an unseeded mode was used as the light source. The light source is able to provide light pulses with the following parameters: pulse energy >0.1mJ; center wavelength, $800 \mathrm{~nm}$; bandwidth, $30 \mathrm{~nm}$; pulse duration, $3 \mathrm{~ns}$; pulse repetition rate (PRR), $10 \mathrm{kHz}$. The output laser pulses were first attenuated to the desired energy with a series of neutral density filters. The beam size was reduced with a beam reducer to fit the aperture of the optical components. The laser pulses were then coupled into the source arm of a $2 \times 2$ single-mode optical fiber coupler, which forms the basis of a fiber-based Michelson interferometer. The bare-fiber tips of the output arms of the $2 \times 2$ fiber coupler were polished to $8^{\circ}$ 
to reduce back reflection from the glass-air boundaries. In the sample arm, the light output from the fiber was collimated and scanned by an x-y galvanometer scanner. The light was delivered to the eye through the combination of an achromatic lens L1 ( $\mathrm{f}=75 \mathrm{~mm}$ ) and an ocular lens L2 ( $\mathrm{f}=20$ mm, 49322INK, Edmund Optics). The light pulse energy was measured to be $400 \mathrm{~nJ}$ at the surface of the eye.

For the OCT imaging mode, the combined reflected light from the sample and reference arms of the interferometer was detected in the detection arm with a spectrometer, i.e. accomplish the OCT function in the spectral domain. The spectrometer consisted of a $1200 \mathrm{line} / \mathrm{mm}$ transmission grating, an imaging lens ( $\mathrm{f}=150 \mathrm{~mm}$ ), and a line scan CCD camera (AVIIVA EM2 $2 \mathrm{k} \times 14 \mu \mathrm{m}$ pixel size, e2V). The theoretical depth resolution of the OCT mode was $9.4 \mu \mathrm{m}$.

For PAM imaging mode, the induced photoacoustic waves from the sample were detected by a custom-built needle ultrasonic transducer (30 MHz; bandwidth: 50\%; active element diameter: $0.4 \mathrm{~mm}$ ). When imaging the retina the ultrasonic transducer was placed in contact with the eyelid coupled with ultrasound coupling gel. The detected photoacoustic (PA) signals were first amplified by $40 \mathrm{~dB}$, and then digitized and streamed to the computer by a high speed 14-bit digitizer (PCI5122, National Instruments) at a sampling rate of $100 \mathrm{MS} / \mathrm{s}$. Synchronization among the output of the laser pulse, scanning of the galvanometer, acquisition of the OCT interfering spectrum, and acquisition of PAM data was achieved by a multi-channel digital delay generator (DG645, Stanford Research Systems). A synchronization signal from the light source was used to trigger the delay generator. The outputs of the delay generator served as the sample clock of an analog output board (PCI-6731, National Instruments), which controlled the x-y galvanometer scanner, triggered the image acquisition board for the CCD camera of the spectrometer and digitizer for acquisition of the PAM signals. 
Figure 3.2 shows the measured spectrum of the light source and the corresponding pointspread-function (PSF) of the OCT system. The PSF was measured with a mirror as the sample and the path length difference was set at $1 \mathrm{~mm}$. The measured depth resolution is $9.9 \mu \mathrm{m}$ in air, which well agreed with the theoretical prediction.
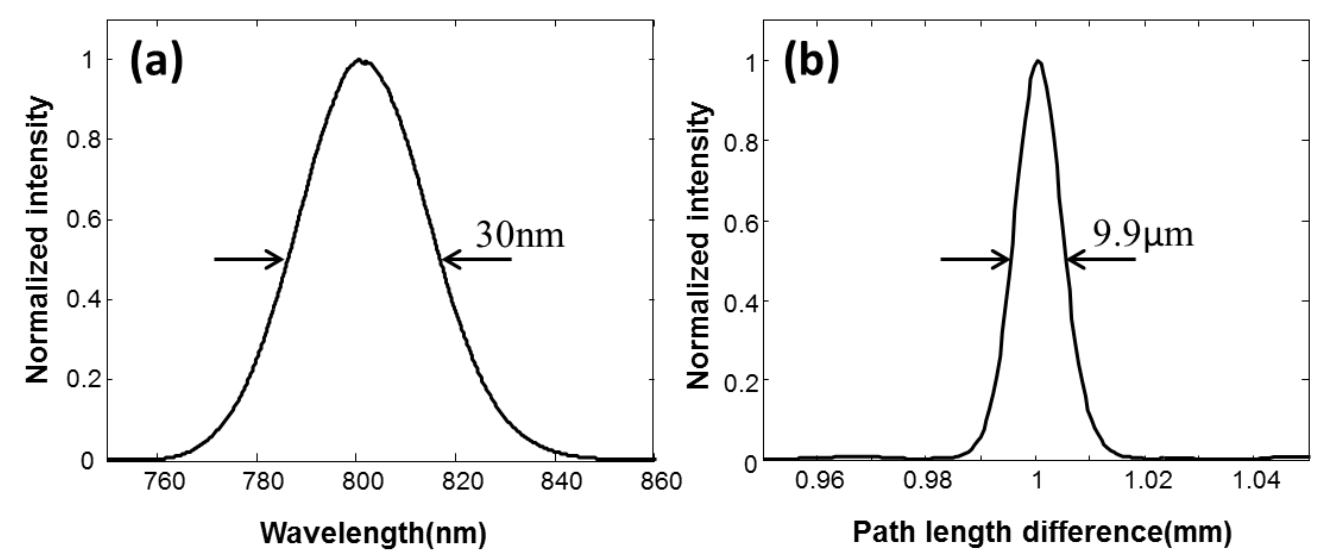

Figure 3.2 Performance of the OCT mode. (a) The measured spectrum of the light source. (b) The calculated PSF of the OCT subsystem.

\subsubsection{Animal Imaging}

To test the capabilities of the system for imaging biological tissues in vivo, we imaged the eyes of Long Evans rats (body weight: 600 g, Charles Rivers). The animals were anesthetized by intraperitoneal (IP) injection of a cocktail containing Ketamine (54 mg/kg body weight) and Xylazine (6 mg/kg body weight). Then we dilated the rats' pupils with $0.5 \%$ tropicamide ophthalmic solution. Artificial tears were applied to the animals' eyes every two minutes to prevent dehydration of the cornea and cataract formation. After anesthetization, the rats were restrained in an animal mount, which was fixed on a five-axis platform. All experiments were performed in compliance with the guidelines of the Florida International University’s Institutional Animal Care and Use Committee. 


\subsection{Results}

\subsubsection{OC-PAM Images}
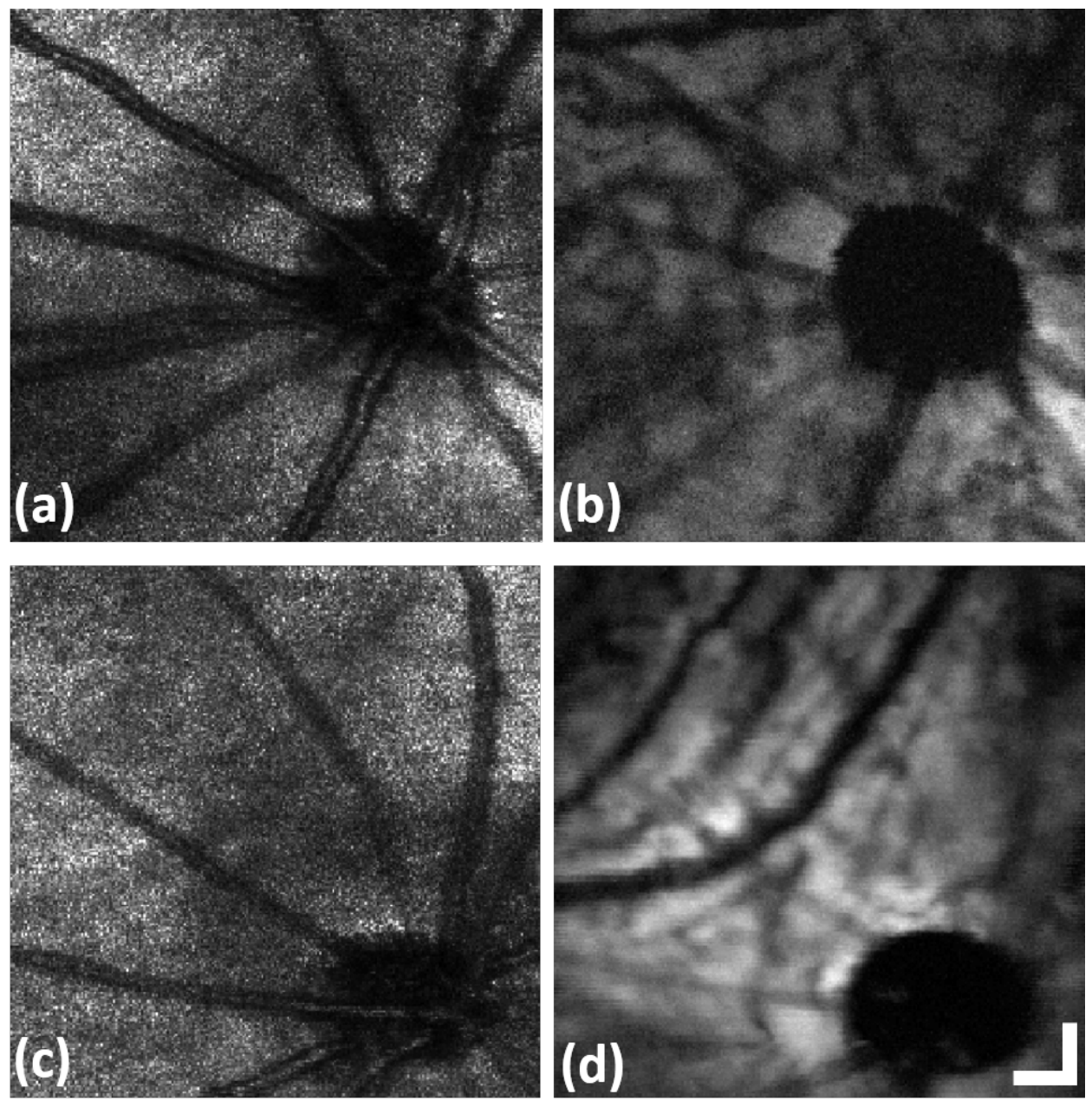

Figure 3.3 The simultaneously acquired OCT and PAM images of two rat eyes. (a) and (c): OCT fundus images generated from the acquired 3D OCT datasets. (b) and (d): The maximum-amplitude-projection (MAP) of the PA datasets. Bar: $200 \mu \mathrm{m}$

Figure 3.3 shows the simultaneously acquired OCT and PAM images of a rat eye. Figure 3.3 (a) and (c) show the OCT fundus images generated from the acquired 3D OCT datasets of rat 1 and rat 2, respectively.[35] Figure 3.3 (b) and (d) show the maximum-amplitude-projection (MAP) of the photoacoustic datasets of rat 1 and rat 2, respectively. All 3D datasets consist of $256 \times 256$ A-lines (depth scans), covering a retinal area of $1.7 \times 1.7 \mathrm{~mm}^{2}$. Since both OCT and PAM images 
are generated from the same group of photons, they are automatically and precisely registered in the lateral directions, which is evidenced in the figure. The lateral positions of each pixel of OCT and PAM image are determined by the scanning of the light pulse. Each light pulse contributes to both one A-line of OCT and one A-line of PAM.

We can see that the projected OCT and PAM images show significantly different features of the retina although the corresponding signals were generated from the same group of photons. The OCT fundus image shows clearly the structure of the major retinal blood vessels while the contrast of these blood vessels to the background of the PAM image is so small that they are barely recognizable. In the projected PAM image we also see thinner blood vessel-like shadows in areas between the major retinal blood vessels and these shadows are absent in the OCT fundus image. Our previous retinal imaging results of pigmented rats with visible light PAM have shown that except the retinal blood vessels the photoacoustic signals also come from melanin in the RPE cells.

In the current PAM image if the photoacoustic signals come from the RPE layer as in the case of visible light PAM, one would expect that the shadows are casted by the retinal vessels because only the retinal vessels are in front of the RPE layer and can block the illuminating light. However, in the wavelength range of the light source the light absorption by hemoglobin in the retinal vessels is weak. As a result, although the major light absorbers are melanin either in the RPE cells or in the choroid, the shadows were unlikely casted by the small retinal vessels.

A careful analysis of the B-scan images of OCT and PAM indicated that the shadows are corresponding to the locations of choroidal vessels, as shown in Figure 3.4. The simultaneously acquired OCT [Figure 3.4(a-b)] and PAM [Figure 3.4(c-d)] B-scan images, each of 2048 A-lines, clearly show that the shadows in the PAM image correspond well with choroidal vessels in the OCT image [Figure 3.4, vertical lines]. Figure 3.4(a) and (c) are the images of rat 1. Figure 3.4(b) 
and (d) are the images of rat 2. A shadow casted by a retinal blood vessel [Figure 3.4(a), red arrow] also casted a shadow in the corresponding PAM image [Figure 3.4(c)].
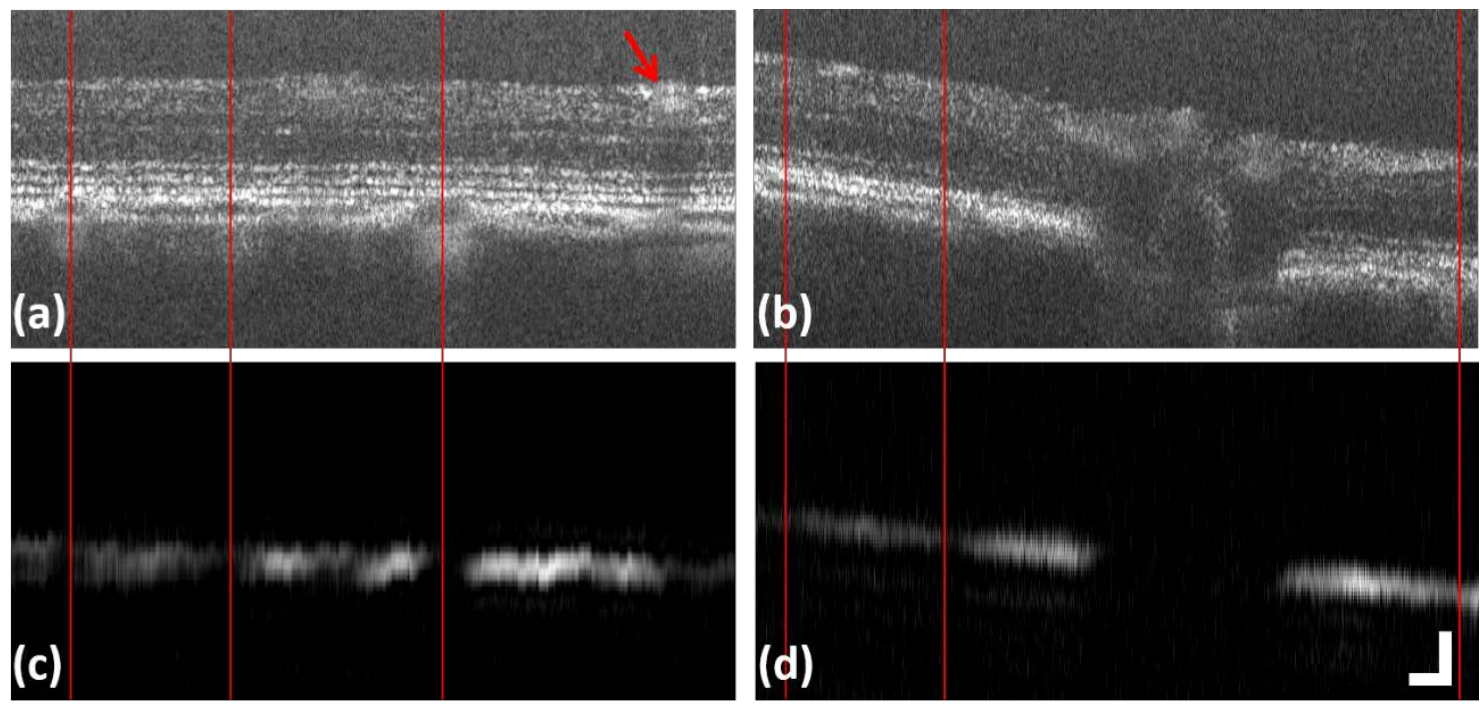

Figure 3.4 The simultaneously acquired OCT and PAM B-scan images. The red arrow indicates retinal blood vessel. Bar: $100 \mu \mathrm{m}$

\subsubsection{Histological Study}

To further verify that many of the shadows in the PAM image were choroidal vessels, we performed histological analysis on one of the rat eyes. Cross sections of the eye were obtained with a cryostat. From the histological image [Figure 3.5(a) and Figure 3.5(c-e)] it is clear that the concentration of melanin in the choroid is much higher than that in the RPE, indicating that choroidal melanin contributes much more to the absorption contrast than the RPE melanin in PAM images. The absence of melanin in choroidal blood vessels and concentrated melanin in the surrounding tissue should produce high contrast images in PAM with weak absorption at the sites where the choroidal blood vessels locate and much stronger signal in surrounding tissue. These features are in good agreement with the weak signals of blood vessels in photoacoustic image. In addition, the choroidal blood vessels in the histological image [Figure 3.5(a), red arrows] match 
well the choroidal vessels in the OCT cross-sectional image [Figure 3.5(b), red arrows]. These results provide strong evidence that the shadows in PAM image are the distribution of choroidal blood vessels.
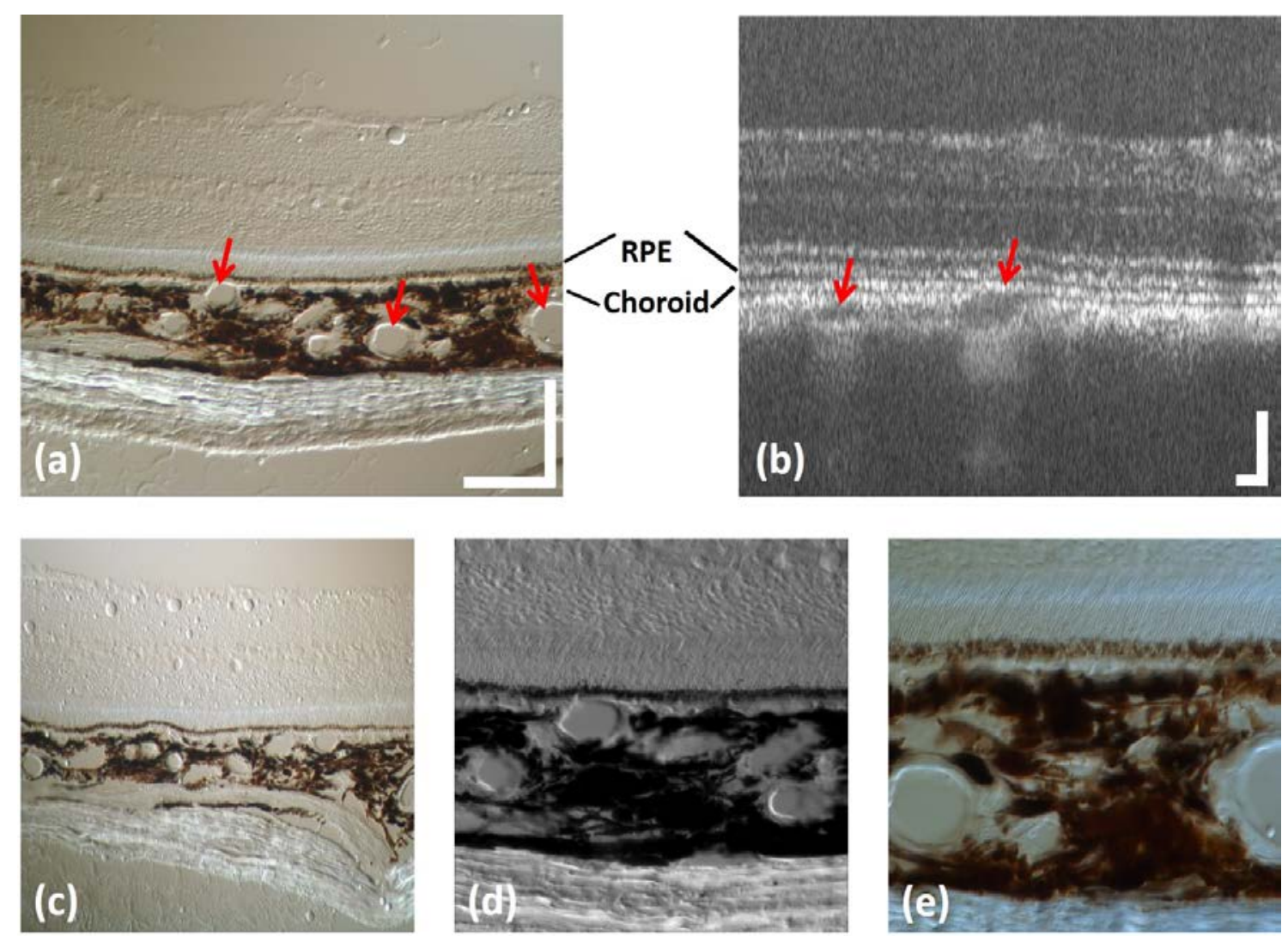

Figure 3.5 Comparison of the histologic image (a) with the OCT cross-sectional image (b) of rat retina. Bar: $100 \mu \mathrm{m}$. (c-e) Additional histologic images with different areas of $593 \times 444 \mu \mathrm{m}^{2}, 213 \times 213 \mu \mathrm{m}^{2}$ and $195 \times 147 \mu \mathrm{m}^{2}$, respectively

Studies by other groups have shown that differences exist between the melanin concentrations in the RPE between rodents and primates (results not published). The rat's RPE layer could not be imaged by photoacoustic microscopy whereas the RPE layers of monkey and primes could be. We haven't performed such study yet but we believe this is what we can further prove with this system in the future. 


\subsection{Discussion}

The maximum permissible exposure (MPE) at $800 \mathrm{~nm}$ for the eye is calculated to be $305 \mathrm{~nJ}$ for single pulse illumination according to the ANSI laser safety standard. When multiple light pulses overlap in the retina in the case of short scanning range, the MPE will be lower. As a result, the pulse energy of $\sim 400 \mathrm{~nJ}$ in this experiment is higher than the MPE due to the sensitivity limitation of the ultrasonic transducer. The pulse energy can be lowered when a transducer with better sensitivity is available. Although the high pulse energy used in the experiments, we didn't see any damage to the rat retina after at least ten times of imaging experiments in a period of 6 months.

Comparing with PAM using a monochromatic light source, the broader bandwidth in OCPAM may have a negative effect on the signal intensity of PAM imaging depending on the absorption spectrum of the absorber. Because the absorption coefficient of RPE melanin decreases monotonically with wavelength in the visible and NIR spectral range, as long as the spectrum of the light source is symmetric the bandwidth of the light source should not affect the signal-to-noise ratio. Achromatic aberration of the optical system including that of the eye may affect the lateral resolution of both the two imaging modes and thus the energy deposition in the absorbers. This may affect the signal intensity of PAM. In the current experiment the bandwidth of the light source is only $30 \mathrm{~nm}, \Delta \lambda / \lambda=3.8 \%$, the effect can be neglected.

The PRR of the light source is $10 \mathrm{kHz}$, i.e. the imaging speed is 10,000 lines/s, which is not as high as conventional spectral-domain OCT. Increasing the PRR can increase the imaging speed. However, increasing the PRR will also increase the average power of the light source, which may cause laser safety issues in practical applications when there is pulse overlapping in the retina, i.e. repetitive exposure in a single spot. Thus, in practical applications there is a tradeoff between pulse 
energy and PRR. In all situations an ultrasonic transducer with high sensitivity is a key for reducing the laser pulse energy, thus makes it safe for eye imaging.

We can see in the NIR spectrum in our current system configuration the major contribution of the PAM signal is from melanin in the RPE cells. Blood vessels appear dark in these NIR PAM images in contrast to the bright images in a visible light PAM[75]. To image the retinal or choroidal blood vessels with PAM a transducer with better sensitivity is necessary so that the weak photoacoustic signals generated from the blood vessels can be picked up. Using contrast agents to increase the absorption in the NIR is another possible solution.

\subsection{Conclusion}

In conclusion, we have demonstrated the feasibility of an OC-PAM system working in the near-infrared. By using a single pulsed broadband NIR light source, OC-PAM can image the scattering and absorption contrasts simultaneously. This system can provide both deep penetration depth as conventional OCT and melanin-specific absorption contrast, which is potentially suitable for human ophthalmic applications. 


\section{CHAPTER 4. OPTICAL COHERENCE PHOTOACOUSTIC MICROSCOPY (OC-PAM) WITH AN INTENSITY-MODULATED CONTINUOUS-WAVE BROADBAND LIGHT SOURCE[107]}

\subsection{Introduction}

Optical coherence tomography (OCT)[1, 2] and Photoacoustic microscopy (PAM)[37-41] are two noninvasive high-resolution three-dimensional biomedical imaging modalities based on different contrast mechanisms. OCT detects the light backscattered from a biological sample either in the time or spectral domain using an interferometer to form an image. The backscattered light carries information of different aspects of light tissue interactions, thus OCT can provide a variety of contrasts for imaging such as intensity, polarization, and Doppler shift. OCT is playing an indispensable role in ophthalmic clinics for the diagnosis of ocular diseases. OCT is also a very promising candidate for imaging the functions of the retina.[108, 109]

PAM is a branch of photoacoustic imaging. By detecting the light induced acoustic waves to form an image, PAM is sensitive to optical absorption, thus can provide absorption-specific imaging contrast. The most prominent application of PAM is to image the micro-vasculature of biological samples. In ophthalmic applications PAM is able to image the retinal vasculature and the melanin distribution in the retinal pigment epithelium (RPE) in vivo.[39] By using multiwavelength illumination, PAM is able to image the blood vessel oxygenation, which provides functional information of a living biological object.[37]

Due to their complementary contrast mechanisms OCT and PAM are suitable for being combined to achieve multimodal imaging. Previous studies have demonstrated that such a multimodal imaging technique is able to provide both the optical scattering and optical absorption information of a subject simultaneously. [39, 67, 70-73] However, all the imaging systems in those studies employed two different light sources for OCT and PAM, due to their different needs: to 
achieve good depth resolution OCT requires a broadband light source, usually in the near-infrared (NIR), whereas a short-pulsed laser is used for PAM to induce transient thermal-elastic expansion in the sample. Simultaneous multimodal OCT and PAM imaging with the same group of photons is achievable by using a pulsed broadband light source either in the visible[75] or NIR[106]. This technique of integrating OCT and PAM with the same light source was named "optical coherence photoacoustic microscopy (OC-PAM)” because OCT and PAM are an integral part of the technology. Previously, OC-PAM systems used a pulsed broadband light source in which each light pulse generates one depth scan (A-line) for both OCT and PAM. In this configuration, the OCT image quality highly depends on the pulse-to-pulse stability of the light source without averaging. In addition, laser safety is always a concern for in vivo applications, especially for eye imaging with a pulsed light source. Therefore, a continuous wave (CW) light source is desired for OC-PAM applications. CW light source has been used for photoacoustic imaging. Murray and Balogun[110] first reported a photoacoustic microscopy illuminated with an intensity-modulated CW laser. The ultrasonic waves were detected with a narrow-band ultrasonic transducer and a lockin amplifier. Maslov and Wang also reported a PA imaging system using an intensity-modulated CW laser diode and demonstrated the capability of imaging biological tissue such as rabbit ear.[111]

In this chapter, our latest progress on OC-PAM with an intensity-modulated superluminescent diode (SLD), a light source commonly used in OCT, is reported. The system was successfully tested on imaging biological tissues both ex vivo and in vivo. 


\subsection{Methods}

\subsubsection{Experimental System}

The experimental system is schematically shown in Figure 4.1. A commercial intensitymodulated SLD module (IPSDS0815-0311, Inphenix Inc.; output power: $6.3 \mathrm{~mW}$; center wavelength: $850 \mathrm{~nm}$; bandwidth: $33 \mathrm{~nm}$ ) was used as the light source. The modulation frequency was set at $2.25 \mathrm{MHz}$ to match the center frequency of the available commercial ultrasonic transducer. A 100\% modulation depth was achieved by applying a 2.2-V sinusoidal signal from a function generator. The modulated output light was coupled into the source arm of a $2 \times 2$ singlemode optical fiber coupler, which forms the basis of a fiber-based Michelson interferometer. In the sample arm, the light exiting the fiber was first collimated and the beam was expanded to fit the aperture of the objective lens (Microspot focusing objectives, $\mathrm{f}=40 \mathrm{~mm}, \mathrm{LMH}-5 \mathrm{X}-532$, Thorlabs). The light power was measured to be $2.3 \mathrm{~mW}$ at the surface of a sample. In the reference arm, a BK7 glass plate was used to compensate for the group-velocity dispersion mismatch between the two interfering arms.

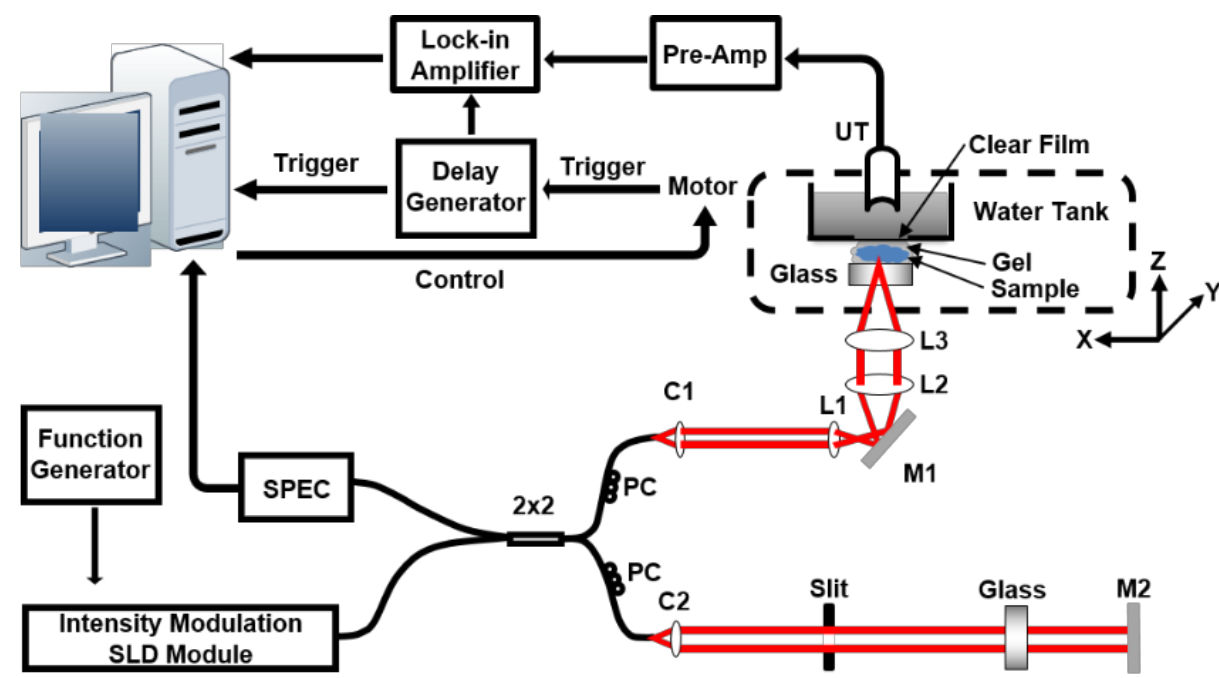

Figure 4.1 Schematic of the experimental system of a fiber-based OC-PAM with an intensity-modulated light source. L1, L2, L3: achromatic lens; PC: polarization controller; UT: ultrasonic transducer; M1, M2: mirror; C1, C2: collimator. 
For OCT imaging, the combined reflected light from the sample and reference arms of the interferometer was detected in the detection arm with a spectrometer. The spectrometer consisted of a 1200 line/mm transmission grating, an imaging lens ( $\mathrm{f}=150 \mathrm{~mm}$ ), and a line scan CCD camera

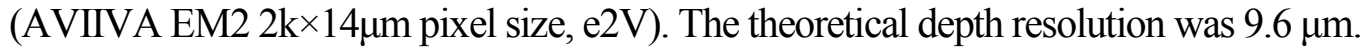

For PAM imaging, the induced ultrasonic waves from the sample were detected by a commercial 2.25 MHz focused ultrasonic transducer (V323-SM, focal length: 6mm, Panametrics) in transmission mode. The transducer was immersed in a water tank filled with distilled water. A window was opened at the bottom of the water tank and was sealed with a transparent polyethylene membrane. For imaging, a sample is placed under the window between the polyethylene membrane and a glass cube, and acoustically coupled with ultrasonic coupling gel. The components within the dashed-box in Figure 4.1 were translated by a 2-axis motorized stage (UniSlide, Velmex, Inc.). The PA signals were first amplified by two pre-amps with a total gain of $96 \mathrm{~dB}$. The amplified PA signal was detected by a lock-in amplifier (SR844, Stanford Research Systems) and then digitized by a high speed 12-bit digitizer (PCI-5124, National Instruments) at a sampling rate of $100 \mathrm{MS} / \mathrm{s}$. Synchronization among the mechanical scan of the motor, acquisition of the OCT interfering spectrum, and acquisition of PAM data was achieved by a multi-channel digital delay generator (DG645, Stanford Research Systems). At the end of each moving step, the TTL pulse output from the motor controller was used to trigger the delay generator. The delay generator then generated two $30 \mathrm{~ns}$ pulses, one of which was delayed by $30 \mathrm{~ms}$. The delayed output was used to trigger the digitizer for acquisition of the PAM signal and the other triggered the image acquisition board for the OCT spectrometer. The exposure time of the CCD camera for the spectrometer was set to $36 \mu \mathrm{s}$. At each scanning position, the digitizer acquired 100 data points at 
a sampling rate of $100 \mathrm{MS} / \mathrm{s}$. These 100 data points were averaged to generate one pixel of the PAM image.

\subsubsection{In vivo and ex vivo Imaging}

To test the capabilities of the system for imaging biological tissues in vivo, the ears of Swiss Webster mice (body weight: 28 g, Charles River) were imaged. The animals were anesthetized by intraperitoneal (IP) injection of a cocktail containing Ketamine (54 mg/kg body weight) and Xylazine (6 mg/kg body weight). All experiments were performed in compliance with the guidelines of the Florida International University’s Institutional Animal Care and Use Committee.

Since the optical absorption of hemoglobin is much weaker in the NIR than that in the visible spectrum, gold nanoparticles[112, 113] have been used as contrast agents to enhance the photoacoustic signals for vasculature imaging. Gold nanorods (GNRs) have been proved to be an effective contrast agents for photoacoustic imaging[114] of inflammatory responses[115], sentinel lymph node[116] and tumor in vivo[117, 118]. In our experiments, GNRs (D12M-850-250, Absorption peak: $850 \mathrm{~nm}$, Nanoparz) were injected (70 uL per mouse) via tail vein.

To verify the capability of the system for imaging ocular tissues, imaging was carried out ex vivo with porcine eye tissues (Animal Technologies, Inc.) in which a small piece of the posterior eye wall containing the pigment epithelium (RPE), the choroid, and the sclera was used.

\subsection{Results}

\subsubsection{System Performance}

Figure 4.2 shows the measured spectrum of the light source and the corresponding pointspread-function (PSF) of the OCT system. The PSF was measured with a mirror as the sample and 
the path length difference was set at $1 \mathrm{~mm}$. The measured depth resolution is $9.4 \mu \mathrm{m}$ in air, a value in good agreement with theoretical calculations.
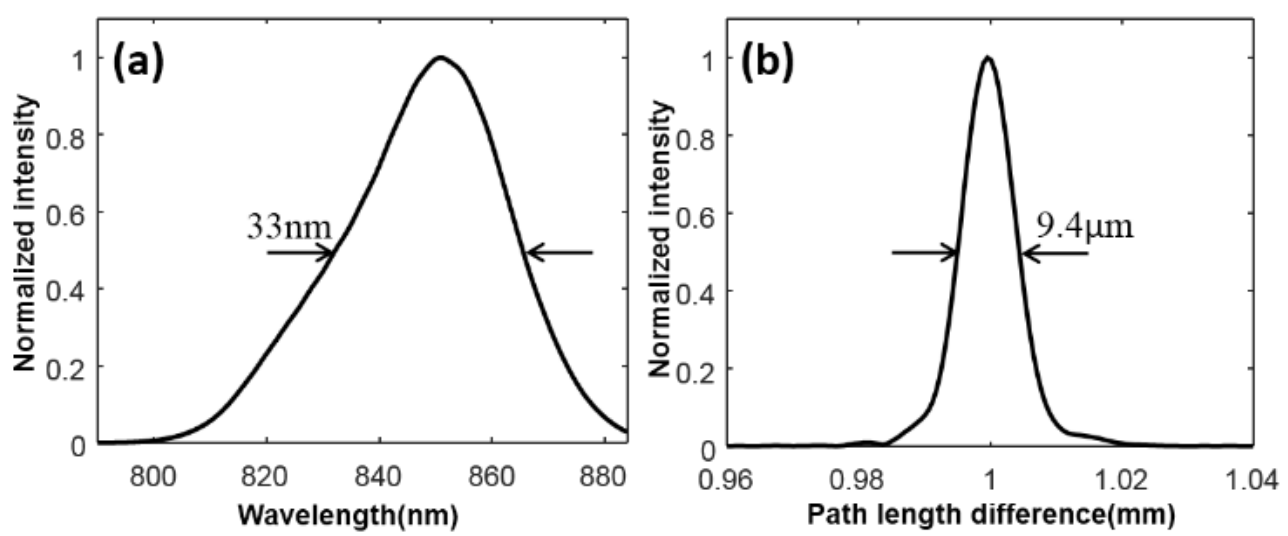

Figure 4.2 Performance of the OCT. (a) The measured spectrum of the light source. (b) The calculated PSF of the OCT subsystem.

(a)

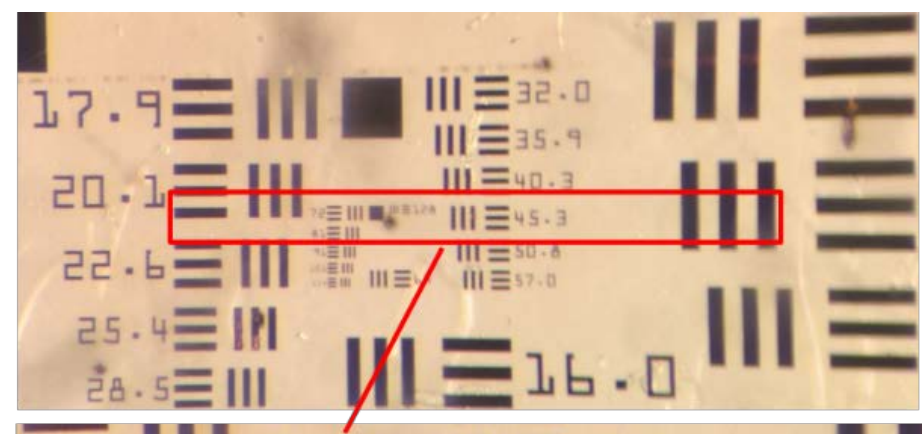

(b)

(c)

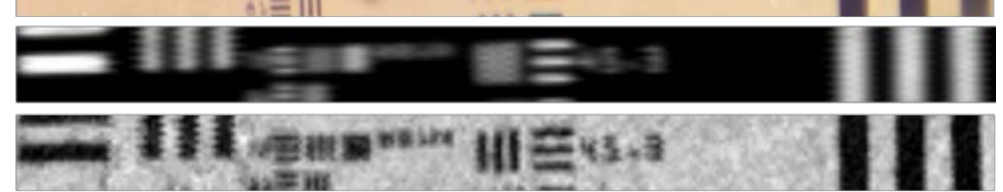

Figure 4.3 Images of USAF 1951 resolution target. (a) Microscopic image. (b) Zoomedin microscopic image of the scanned area. (c) PA image. (d) OCT projection image generated from the acquired 3D OCT dataset.

The system was first tested to image an USAF 1951 resolution target (Figure 4.3). Figure 4.3(a) shows a microphotograph of the resolution target taken with a conventional microscope. The red box in Figure 4.3(a) is the area imaged with the OC-PAM system, which is zoomed in and shown in Figure 4.3(b). Figure 4.3(c) shows the PAM image of the imaged area and Figure 4.3(d) shows 
the OCT projection images generated from the acquired 3D OCT dataset. Both of the PAM and OCT images consist of $256 \times 20$ pixels, covering an area of $2.3 \times 0.18 \mathrm{~mm}^{2}$. The PAM image indicates that the minimum visible resolution bar is in Group 6 Element 2 with the line width of $6.96 \mu \mathrm{m}$.

\subsubsection{In vivo Imaging on Mouse Ear}
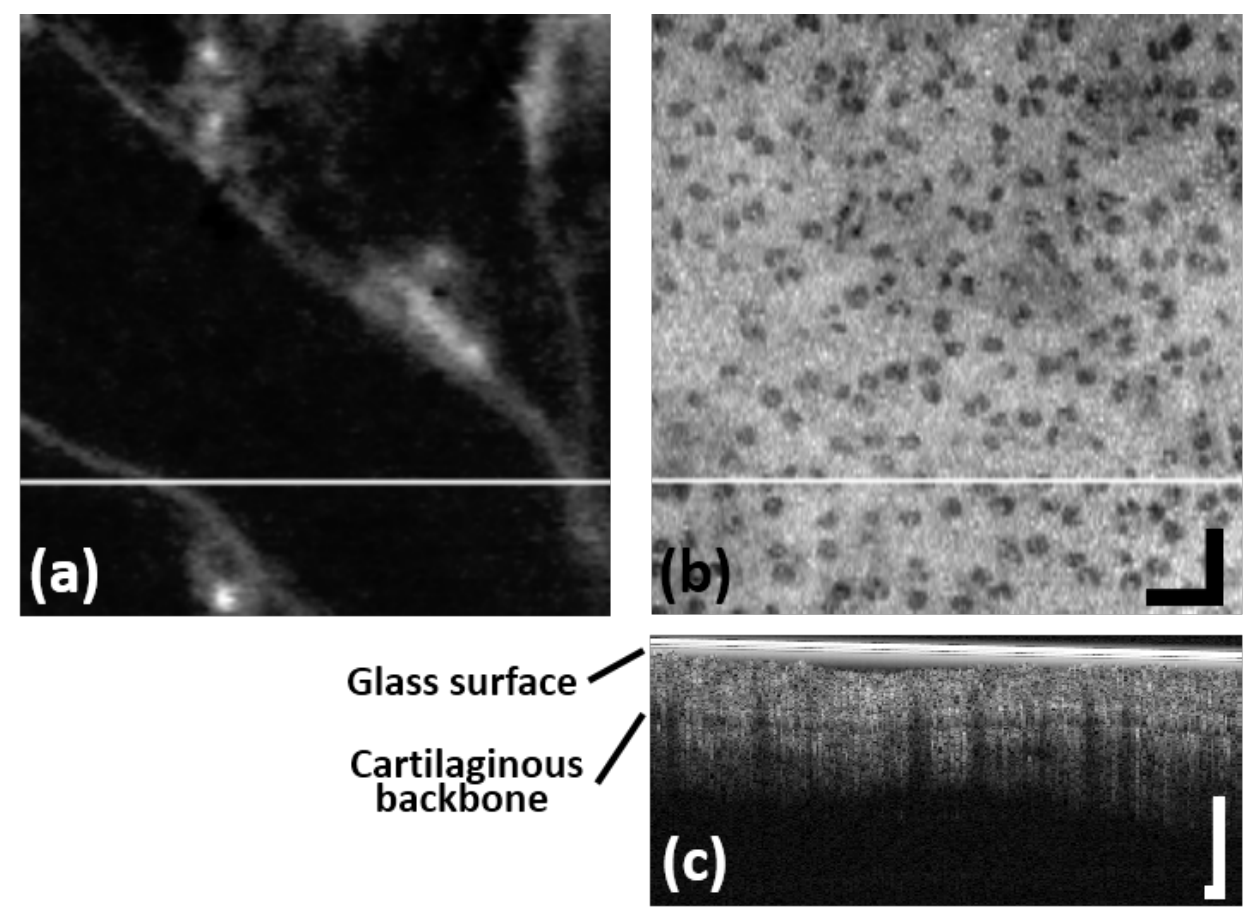

Figure 4.4 Simultaneously acquired PAM and OCT images of a mouse ear in vivo with GNRs injection. (a) PA image. (b) OCT projection images generated from the acquired 3D OCT dataset. (c) OCT cross-sectional image, whose location is marked as a white line on (a) and (b). Bar: $300 \mu \mathrm{m}(\mathrm{a}, \mathrm{b}) 180 \mu \mathrm{m}(\mathrm{c})$

Figure 4.4 shows the simultaneously acquired OCT and PAM images of a mouse ear (Figure 4.4(a): PAM image; Figure 4.4(b): the OCT projection image; Figure 4.4(c): the OCT crosssectional image). GNRs were injected via the tail vein as contrast agent. These images consist of $256 \times 128$ pixels, covering an area of $2.3 \times 2.3 \mathrm{~mm}^{2}$. With the same group of photons, the PAM 
image reveals the blood vessels with good contrast provided by GNRs, whereas the sebaceous glands can be clearly seen with good contrast in the OCT projection image.

\subsubsection{Ex vivo Imaging on Porcine Eye}
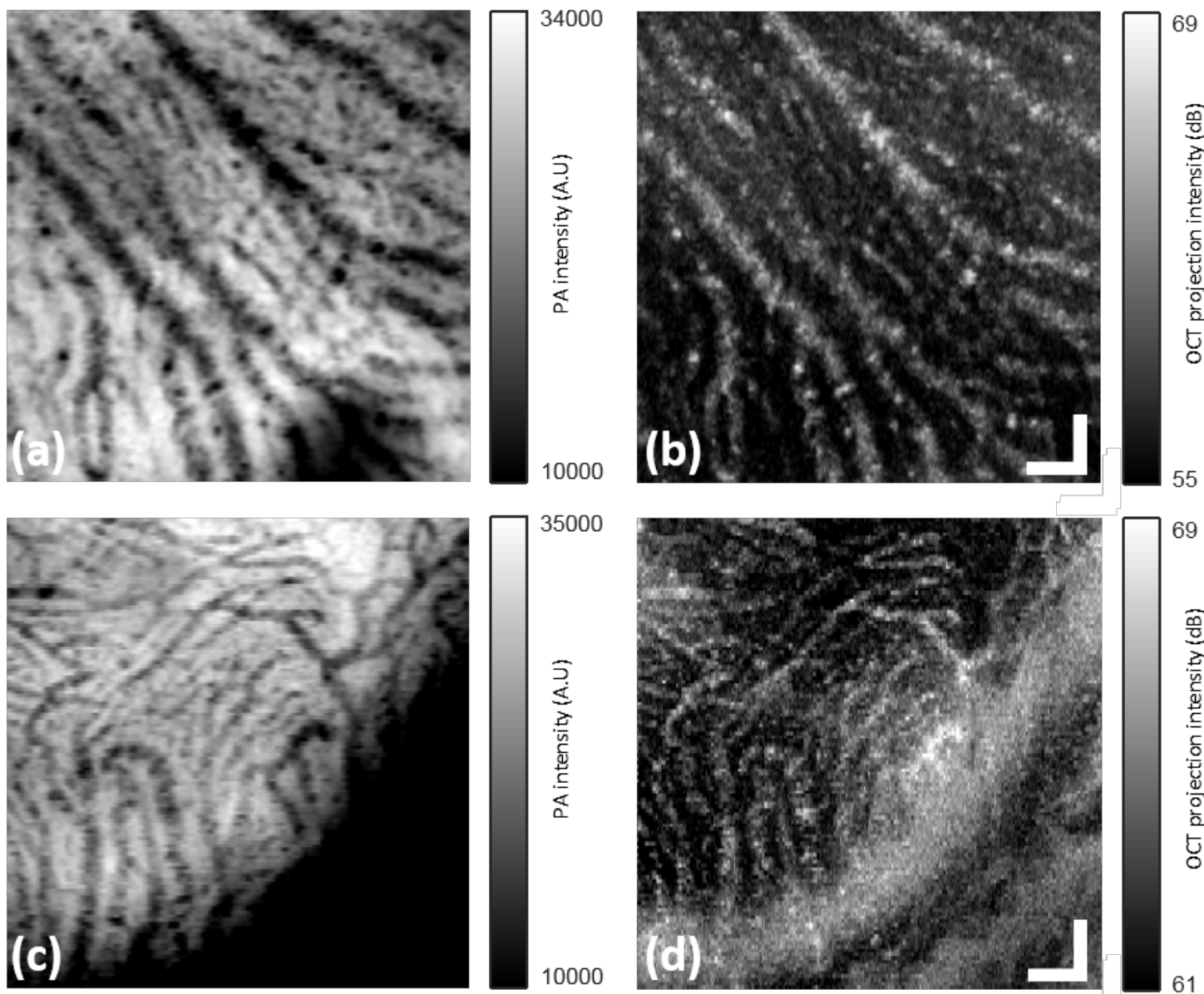

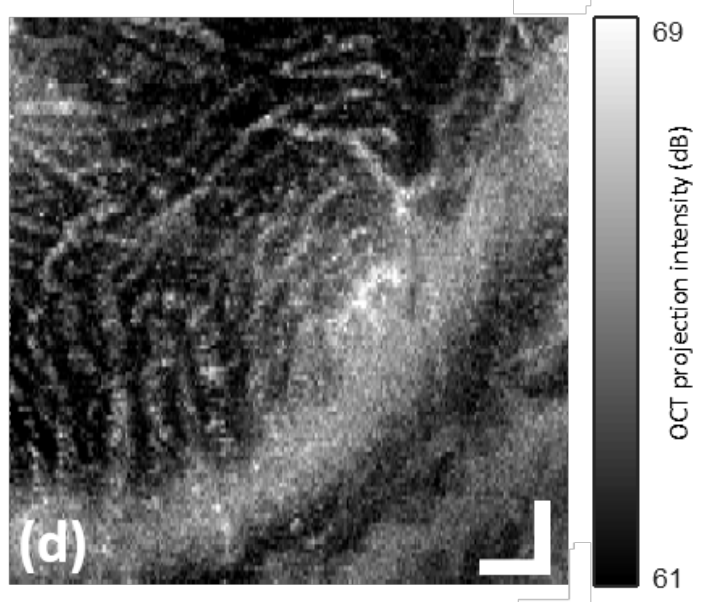

Figure 4.5 Simultaneously acquired PAM and OCT images of a porcine eye ex vivo. (a) and (c) PA images. (b) and (d) OCT projection images generated from the acquired 3D OCT datasets. Bar: Bar: $150 \mu \mathrm{m}(\mathrm{a}, \mathrm{b}) 300 \mu \mathrm{m}$ (c, d)

A porcine eye was imaged ex vivo to show that the system could also image ocular tissues. A piece of the posterior segment of the eye was cut, retina removed, and imaged with the system. Figure 4.5 shows the simultaneously acquired PAM images [Figure 4.5(a) and (c)] and OCT 
projection images [Figure 4.5(b) and (d)]. Figure 4.5(a) and (b) consist of $256 \times 128$ pixels, covering an area of $1.2 \times 1.2 \mathrm{~mm}^{2}$. Figure $4.5(\mathrm{c})$ and (d) consist of $256 \times 128$ pixels, covering an area of $2.3 \times 2.3 \mathrm{~mm}^{2}$. The PAM image has a bright background with dark, blood vessel like patterns, which appear bright in the OCT projection image. In our previous study on rat eye imaging in vivo with pulsed-light NIR OC-PAM system, the dark pattern in the PAM image are confirmed as images of the choroidal blood vessels.[106] Indeed, the cross-sectional OCT image [Figure 4.6(d)] and a microphotography of histological cross-section of the same tissue [Figure 4.6(c)] demonstrate clearly the blood vessel like pattern in Figure 4.5 was choroidal blood vessels (red arrows in Figure 4.6(c) and Figure 4.6(d)).
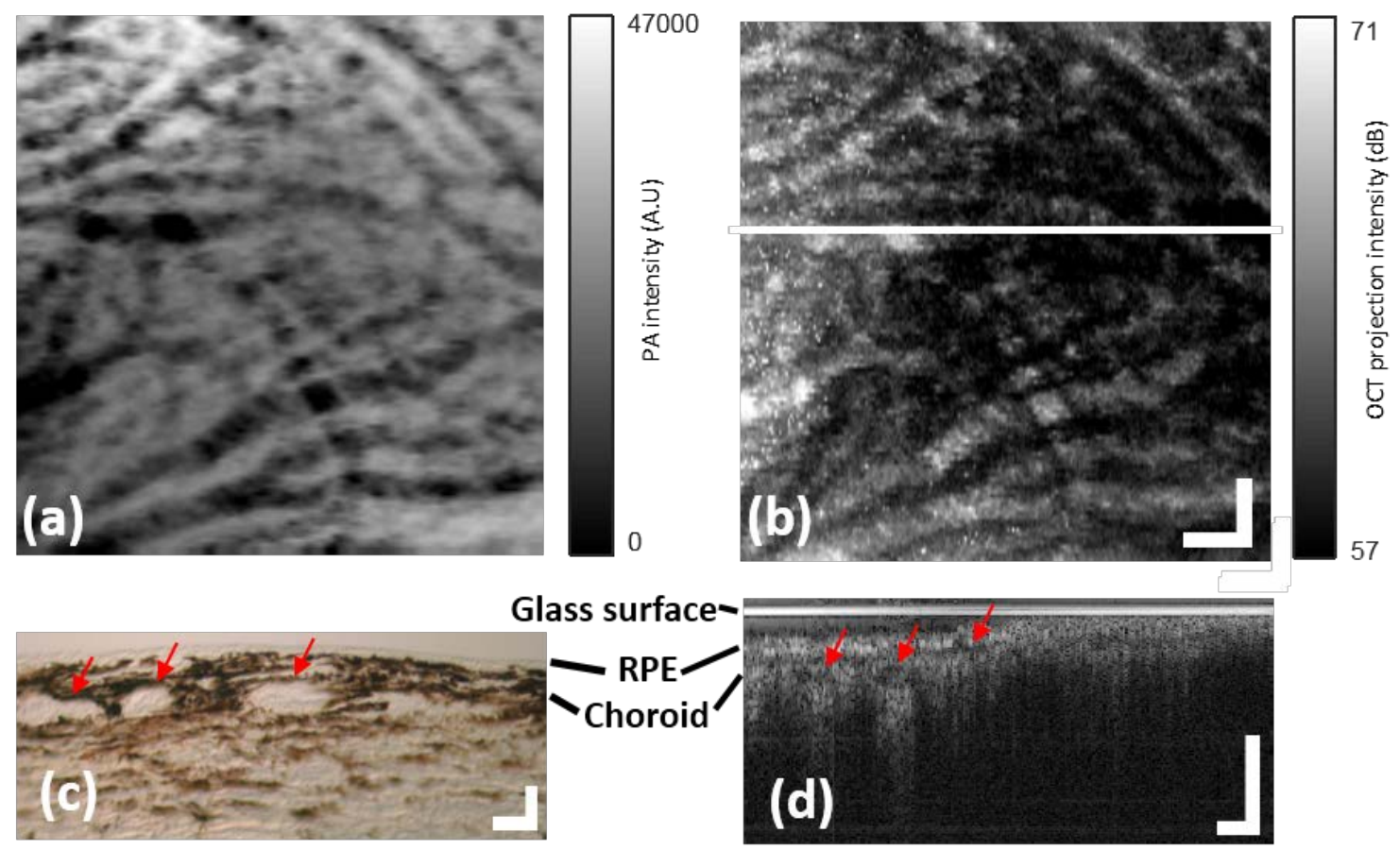

Figure 4.6 Comparison of OCT cross-sectional image and histologic image of porcine eye ex vivo. (a) PA image. (b) OCT projection image, where the white line marks the location of the OCT cross-sectional image shown in (d). (c) Histologic image of porcine eye. (d) OCT cross-sectional image of porcine eye. Bar: $150 \mu \mathrm{m}(\mathrm{a}, \mathrm{b}) 50 \mu \mathrm{m}$ (c) $90 \mu \mathrm{m}(\mathrm{d})$. 
In the porcine eyes, melanin is much more concentrated in the choroid than in the RPE [Figure 4.6(c)], a phenomenon also found in pigmented rat eyes in our previous study[106]. The depth of focus of the ultrasonic transducer is much longer than the thickness of the RPE, thus both the choroid and RPE melanin contributes to the intensity of each pixel of the PAM image. The higher concentration of melanin in the choroid indicates that choroidal melanin is the major contributor to the absorption contrast in PAM images, whereas the absence of melanin in choroidal blood vessels reduces the amount of melanin contributing to the photoacoustic signals in the pixels corresponding to the locations of the vessels, providing the contrast of blood vessels compared with the tissues surrounding the choroidal blood vessels where high melanin concentration is present.

From the OCT cross-sectional image we can see that there were stronger signals from the tissues beneath the choroidal blood vessels, as shown in Figure 4.6(d). The location of the crosssectional image shown in Figure 4.6(d) is marked as a white line in Figure 4.6(b). When we reconstructed the OCT projection image, the choroidal blood vessels appear bright compared to the background. These features are in good agreement with the weak signals of blood vessels in photoacoustic image. In addition, the choroidal blood vessels in the histological image [Figure 4.6(d), red arrows] match well with the choroidal vessels in the OCT cross-sectional image [Figure 4.6(c), red arrows]. These results provide strong evidence that the shadows in PAM image are the distribution of choroidal blood vessels.

\subsection{Discussion}

In this study we can see that the OCT image quality is better than that in the OC-PAM using a pulsed light source because of the much better stability of the commercial SLD light source. To compare the OCT image quality fairly, the pulsed light source used in Chapter 3 and the intensitymodulated broadband SLD light source used in this chapter were combined by a flip mirror and 
coupled into the source arm of fiber-based OCT system as a supplement study of this topic. The same rat was first imaged using the pulsed light source with the flip mirror down and then imaged using the intensity-modulated cw light source by flipping up the mirror.

The OCT cross-sectional images are shown in Figure 4.7. Figure 4.7(a) is the OCT crosssectional image using pulse broadband light source and Figure 4.7(b) is the OCT cross-sectional images using intensity-modulated cw light source. Compared with the OCT image acquired with pulse broadband light source, the one acquired with intensity-modulated cw light source shows stronger signal intensity and better contrast to the background. Furthermore, the layered structures in retina are better resolved in the OCT image acquired with intensity-modulated cw light source.
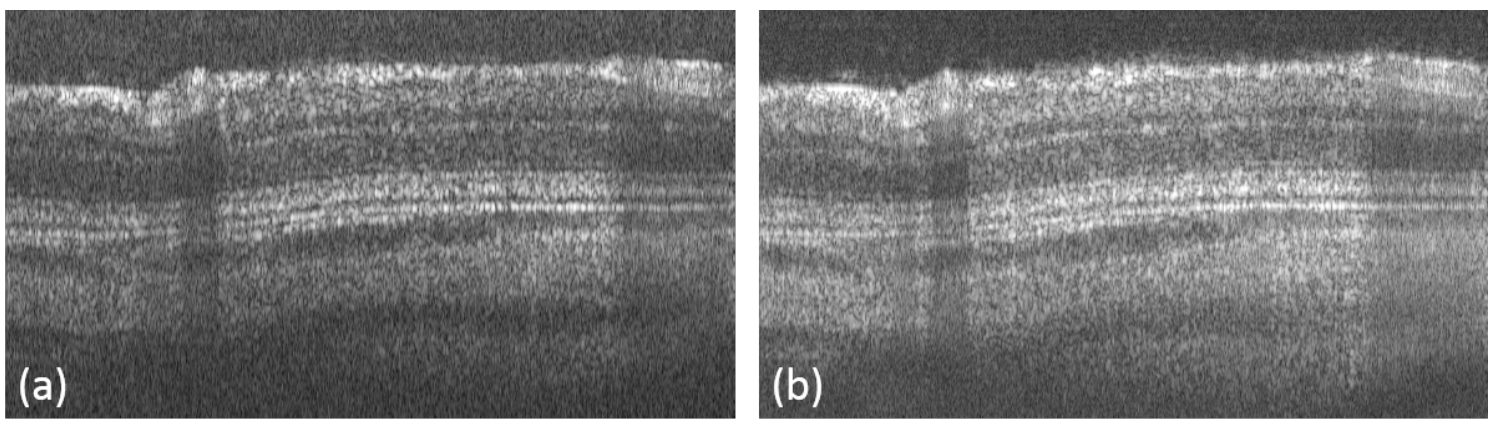

Figure 4.7 Comparison of OCT cross-sectional images acquired by OCT system using pulsed broadband light source and cw light source, respectively. (a) OCT crosssectional images using pulse broadband light source (b) OCT cross-sectional images using cw light source.

However, the PAM image quality in this topic is not very good due to limitation of the ultrasonic transducer sensitivity. As a result, contrast agent needs to be used for vasculature imaging. A possible $20 \mathrm{~dB}$ of magnitude improvement can be expected when a resonant transducer is used[111]. Thus, the system could benefit from using a resonant transducer to image blood vessels without the use of contrast agent. The glass cube at the back of the sample also improved (roughly doubled) the photoacoustic signal by reflecting the ultrasound transmitted through the sample. Our current OC-PAM system is configured in the transmission mode due to limited 
availability of the ultrasonic transducer. The availability of better transducers could allow the system to be configured in the reflection mode more suitable for in vivo imaging.

RPE and choroidal melanin provides good contrast for imaging the eye. Due to the lower optical absorption coefficient in the NIR compared to that in the visible spectrum, thus the much longer penetration depth in the NIR for the illuminating light, it is difficult to distinguish the RPE and choroidal contributions to the PAM signals. Results presented in this chapter and from our previous study [106] described in Chapter 3 clearly indicate that PAM with NIR light source is more suitable for imaging melanin distributions in the choroid. With the high melanin background, the choroidal blood vessels, which lack melanin, can be imaged. This provides a unique capability of PAM for imaging choroidal blood vessels in vivo.

Since the current OCT imaging speed can be as high as 24k A-lines/s with an exposure time of $36 \mu$ s, the imaging speed of the system is mainly limited by the integration time of the lock-in amplifier, thus the speed of PAM, and the mechanical scanning speed of the translational stages. To achieve a steady output, a 30 ms integration time was chosen for the lock-in amplifier. For an image consisting of $256 \times 128$ pixels, it may take more than 17 minutes for data acquisition and about 14 minutes for scanning, thus a total of more than 30 minutes to acquire the whole image. The slow imaging speed may limit the system for in vivo applications such as retinal imaging. Improved scanning speed can be achieved with faster scanning mechanisms such as that used in scanning-laser photoacoustic ophthalmoscope[39]. Increasing the modulation frequency of the light source may help reduce the integration time of the lock-in amplifier, thus improve the imaging speed. 


\subsection{Conclusion}

In conclusion, we have demonstrated the feasibility of an OC-PAM system with an intensitymodulated broadband SLD light source in the NIR. The system was successfully applied to imaging biological samples ex vivo and in vivo. The results show that SLD light source produces better OCT image quality in OC-PAM. Furthermore, the present studies in porcine eyes together with the studies in rat eyes previously provide evident that PAM with NIR light source is a technology capable of imaging the choroidal blood vessels. Finally, our studies point to the need for more sensitive transducers and to improve imaging speed for clinical translation. 


\section{CHAPTER 5. PHANTOM STUDY OF OC-PAM}

\subsection{Introduction}

RPE is a monolayer of pigmented cells between the photoreceptors and choriocapillaris in the eye. There are two major pigments in RPE, melanin and lipofuscin. RPE melanin serves as photoprotective role by absorbing radiation and scavenging free radicals and reactive oxygen species (ROS).[79, 80] On the other hand, lipofuscin is a by-product of phagocytosis of the photoreceptor outer segments, which accumulates with aging of the retina. Excessive levels of lipofuscin accumulation could compromise essential RPE functions and contribute to the pathogenesis of age-related macular degeneration (AMD)[81], a leading cause of blinding diseases in developed countries. Hence, in vivo imaging of melanin and lipofuscin can provide important aging information of the retina, which is important for AMD research and clinical diagnosis.[82]

In the NIR spectrum range, PAM is able to provide melanin-specific imaging of retina and OCT is able to image scattering contrast, which is related to the amount of lipofuscin accumulated in the RPE layer. Since the OCT and PA signals are generated with the same group of photons, the interpreted signal strength would represent the optical properties of the retina in ophthalmologic imaging. Thus, the proposed NIR OC-PAM system is potentially able to provide a unique technique to evaluate the existence of melanin and lipofuscin specifically, which will benefit the diagnosis and research of AMD.

Therefore, to conduct the phantom study and convert the relationship between signal intensities of OCT and PAM to the relationship between the optical scattering property and optical absorption property is very important to evaluate the existence of melanin and lipofuscin in RPE and will be strong evidence for practical application of the proposed NIR OC-PAM system. 
The assumption for this phantom study would be, for a certain phantom with a fixed ratio of the scattering and absorption coefficients, the interpreted signal strength from the acquired images would be the same. This study would enhance the practical application of the NIR OC-PAM system for studying optical properties of biological tissues.

\subsection{Methods}

\subsubsection{Theoretical Model}

As discussed in Chapter 1.1, the detected interference signal is the last tem in Equation 1.91.8 as:

$$
\tilde{I}(\tau)=2 \sum_{n} \sqrt{R_{n}} \Gamma\left[\tau \pm 2\left(\tau_{n}-\tau_{r}\right)\right]
$$

thus, we have:

$$
\tilde{I}^{2}(\tau) \propto R_{n}
$$

The relationship between the reflectivity $\mathrm{R}$ and scattering coefficient $\mu_{s}$ is given as:

$$
\begin{aligned}
R & =\mu_{s} b(g) d z, \\
b(g) & =\int_{\pi-\sin ^{-1} N A}^{\pi} p(\theta) 2 \pi \sin \theta d \theta,
\end{aligned}
$$

where the factor $\mathrm{b}(\mathrm{g})$ is the fraction of the photon that gets back scattered; the phase function, $p(\theta)$ is assumed to be a Henyey-Greenstein phase function.

To simplify the equation, the reduced scattering coefficient $\mu_{s}^{\prime}=\mu_{s}(1-g)$ is used instead, where $\mathrm{g}$ is the anisotropy of scattering. Thus, in OCT imaging we have:

$$
\tilde{I}^{2}(\tau) \propto \mu_{s}^{\prime}
$$

In Chapter 1.2, the solution to the wave equation for the velocity potential can be expressed as Equation 1.13: 


$$
\phi(\boldsymbol{r}, t)=\frac{\beta}{4 \pi \rho_{f} C_{p}} \int_{0}^{t} d t^{\prime} \int d \boldsymbol{r}^{\prime} g\left(\boldsymbol{r}, t \mid \boldsymbol{r}^{\prime}, t^{\prime}\right) H\left(\boldsymbol{r}^{\prime}, t^{\prime}\right)
$$

The heating function for optical illumination may be expressed as:

$$
H(\boldsymbol{r}, t)=\mu_{a}(\boldsymbol{r}) \Phi(\boldsymbol{r}, t)
$$

where $\mu_{a}$ is the absorption coefficient, and $\Phi(\boldsymbol{r})$ is the optical radiation fluence rate. Within the diffusive regime,

$$
\Phi(\boldsymbol{r}) \approx \Phi_{0} e^{-\mu_{e f f} r}
$$

where $\Phi_{0}$ is the illumination fluence, $\mu_{\text {eff }}=\sqrt{3 \mu_{a}\left(\mu_{a}+\mu_{s}^{\prime}\right)}, \mu_{s}^{\prime}$ is the reduced scattering coefficient.

In the OC-PAM system with an intensity-modulated CW light source, the light intensity is expressed as $I=I_{C W}\left[1+\sin \left(\omega_{c} t\right)\right]$, where $I_{C W}$ is the time-averaged light intensity and $\omega_{c}$ is the modulation frequency. If the light intensity is written as $I=I_{0} f(\hat{t})$, where $I_{0}$ denotes the maximum and $\hat{t}$ denotes a dimensionless time $\left(\hat{t}=t / \tau\right.$ or $\left.\omega_{c} t\right)$. In three dimension, the time profile of the photoacoustic wave generated by a long light pulse can be derived as[68, 119]:

$$
p=\mu_{a}(\boldsymbol{r}) \frac{\Phi_{0} e^{-\mu_{e f f} r} \sigma \beta}{4 \pi \theta^{2} C_{P} r} \frac{d}{d \hat{t}} f(\hat{t}),
$$

where $\sigma$ is the optical cross section of the particle, $\theta$ is a pulse width parameter. Thus, the generated PA pressure is proportional to the absorption coefficient $\mu_{a}(\boldsymbol{r})$ :

$$
p(\boldsymbol{r}) \propto \mu_{a}(\boldsymbol{r}) .
$$

\subsubsection{Phantom Design}

Intralipid $^{\mathrm{TM}}$ is an aqueous suspension of lipid droplets that is commonly used in the biomedical optics community. Since it has no strong absorption in the spectra of visible and NIR, Intralipid has 
been widely used as a scattering medium for phantoms that mimic turbid tissues when conducting optical experiments.[120-123]

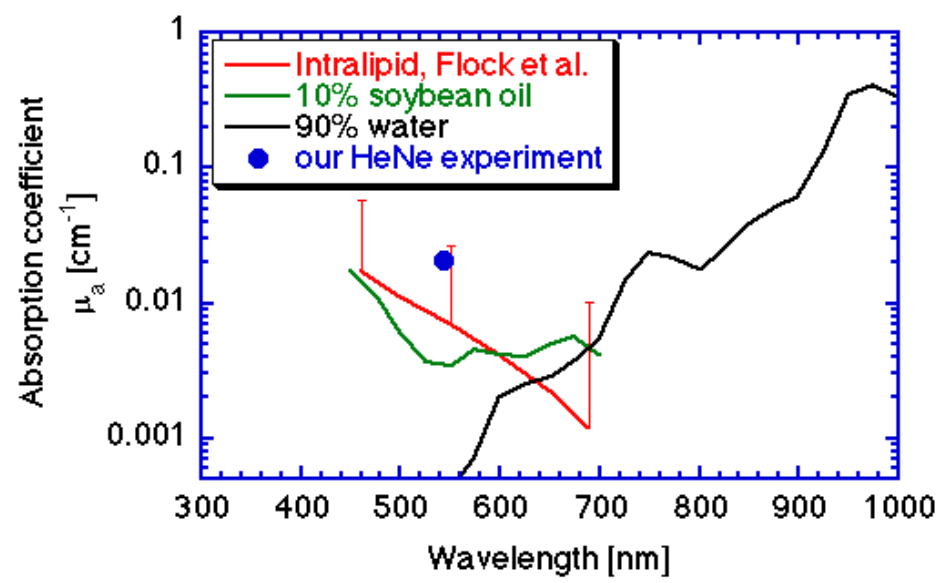

Figure 5.1 The absorption coefficient of Intralipid-10\%.

The optical properties of Intralipid has been studied by several groups.[124-128] A summarized graph of the optical absorption coefficient is shown in Figure 5.1 and scattering properties are shown in Figure 5.2 and Figure 5.3.[129] The absorption coefficient of Intralipid is less than $0.03 \mathrm{~cm}^{-1}$ in NIR spectra, which is much smaller than the absorption of NIR dye, thus it is negligible in this phantom study.
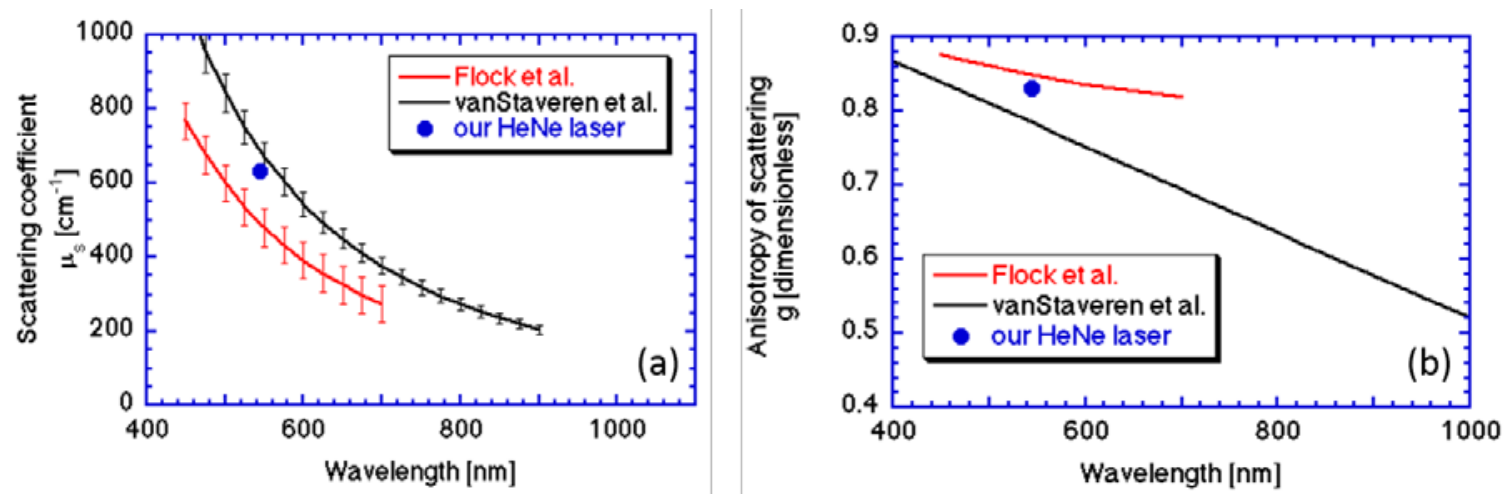

Figure 5.2 The scattering properties of Intralipid-10\%. (a) The scattering coefficient of Intralipid-10\%. (b) The anisotropy of scattering of Intralipid-10\%. 


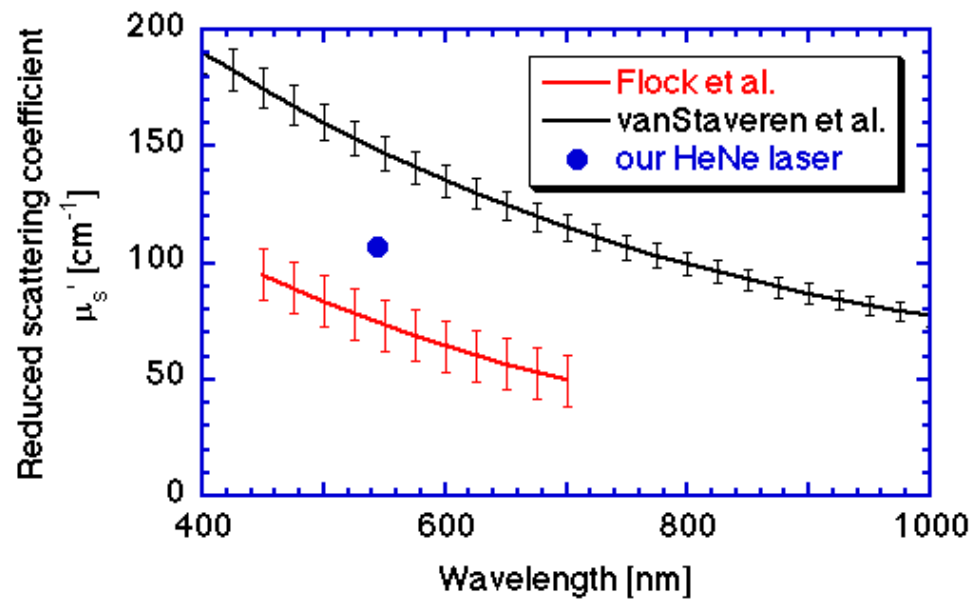

Figure 5.3 The reduced scattering coefficient of Intralipid-10\%.

According to the study of van Staveren et al.[128], the Mie theory approximation of the scattering coefficient of Intralipid-10\% is:

$$
\mu_{s}=2.54 \times 10^{9} \lambda^{-2.4}\left(\mathrm{~cm}^{-1}\right) \text {, }
$$

and the anisotropy of scattering of Intralipid-10\% is:

$$
g=1.1-0.58 \times 10^{-3} \lambda,
$$

where $\lambda$ is the wavelength in nanometers. In our OC-PAM system, the center wavelength is $850 \mathrm{~nm}$, thus the calculated $\mu_{s}$ and $g$ of Intralipid-10\% from Equation 5.10 and Equation 5.11 are $236.7 \mathrm{~cm}^{-1}$ and 0.607 , respectively.

The reduced scattering coefficient of Intralipid-10\% using $\mu_{s}$ and $g$ calculated above is:

$$
\mu_{s}^{\prime}=\mu_{s}(1-g)=93 \mathrm{~cm}^{-1}
$$

NIR869A (QCR Solutions Corp) is a commercial water-soluble absorbing NIR dye specially designed for use in water-based systems. The absorption peak of the NIR869A is $869 \mathrm{~nm}$, as shown in its absorption spectrum in Figure 5.4. The absorptivity of NIR869A is $273 \mathrm{~L} \mathrm{~g}^{-1} \mathrm{~cm}^{-1}$, thus the absorption coefficient $\mu_{\mathrm{a}}$ is $273 \mathrm{~cm}^{-1}$ for concentration of $1 \mathrm{~g} / \mathrm{L}$. 


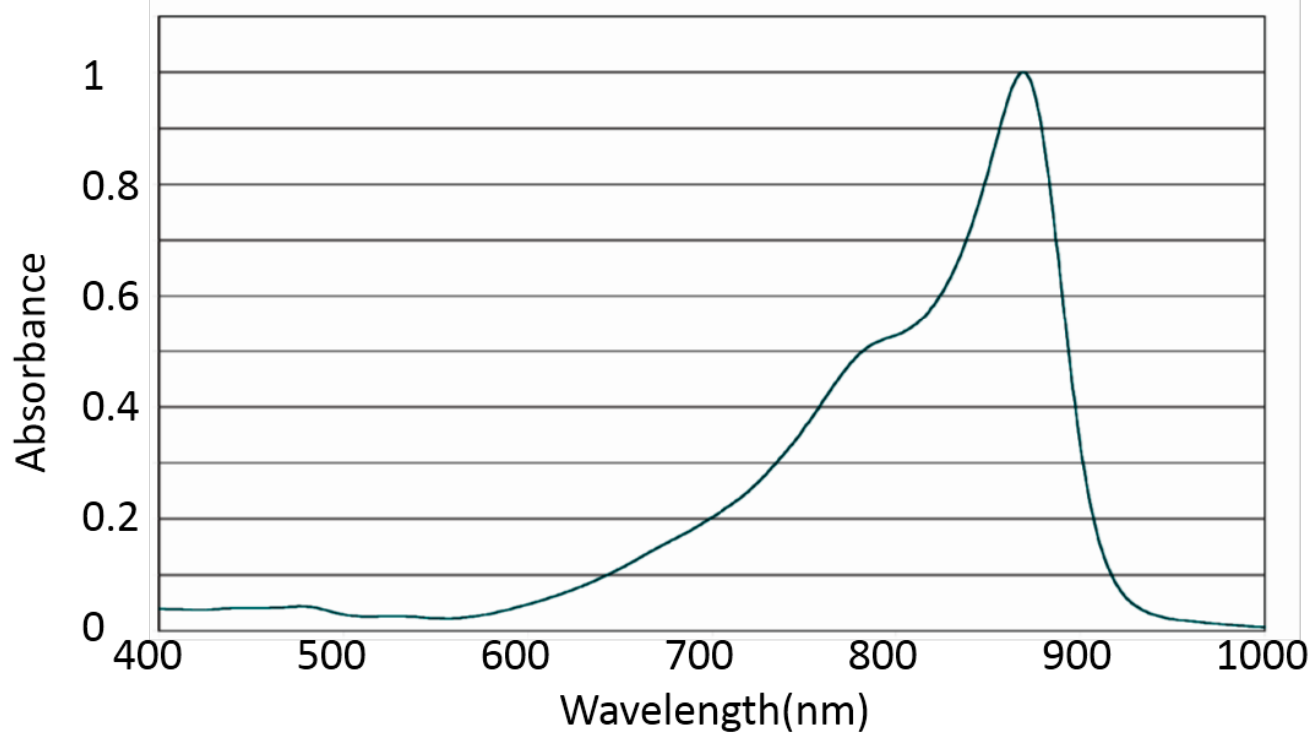

Figure 5.4 The absorption spectrum of NIR869A.

In this phantom study, the Intralipid was used as a scattering substance of lipofuscin phantom and the NIR dye as an absorption phantom of RPE melanin. By determining the optical properties of melanin and lipofuscin, the phantoms will be prepared with different mixture ratios in the range of the similar scattering and absorption coefficients.

The optical properties of melanosome has been studied by several groups.[130-133] A summarized graph of the optical absorption coefficient is shown in Figure 5.5.[77] The absorption coefficient of retinal melanosome can be approximated as[132]:

$$
\mu_{a}=6.49 \times 10^{12} \lambda^{-3.48}\left(\mathrm{~cm}^{-1}\right),
$$

where $\lambda$ is the wavelength in nanometers. Thus, the absorption coefficient at the wavelength of 850 $\mathrm{nm}$ is $414.8 \mathrm{~cm}^{-1}$, which is approximately the same absorption as NIR dye with a concentration of $1.52 \mathrm{~g} / \mathrm{L}$. 


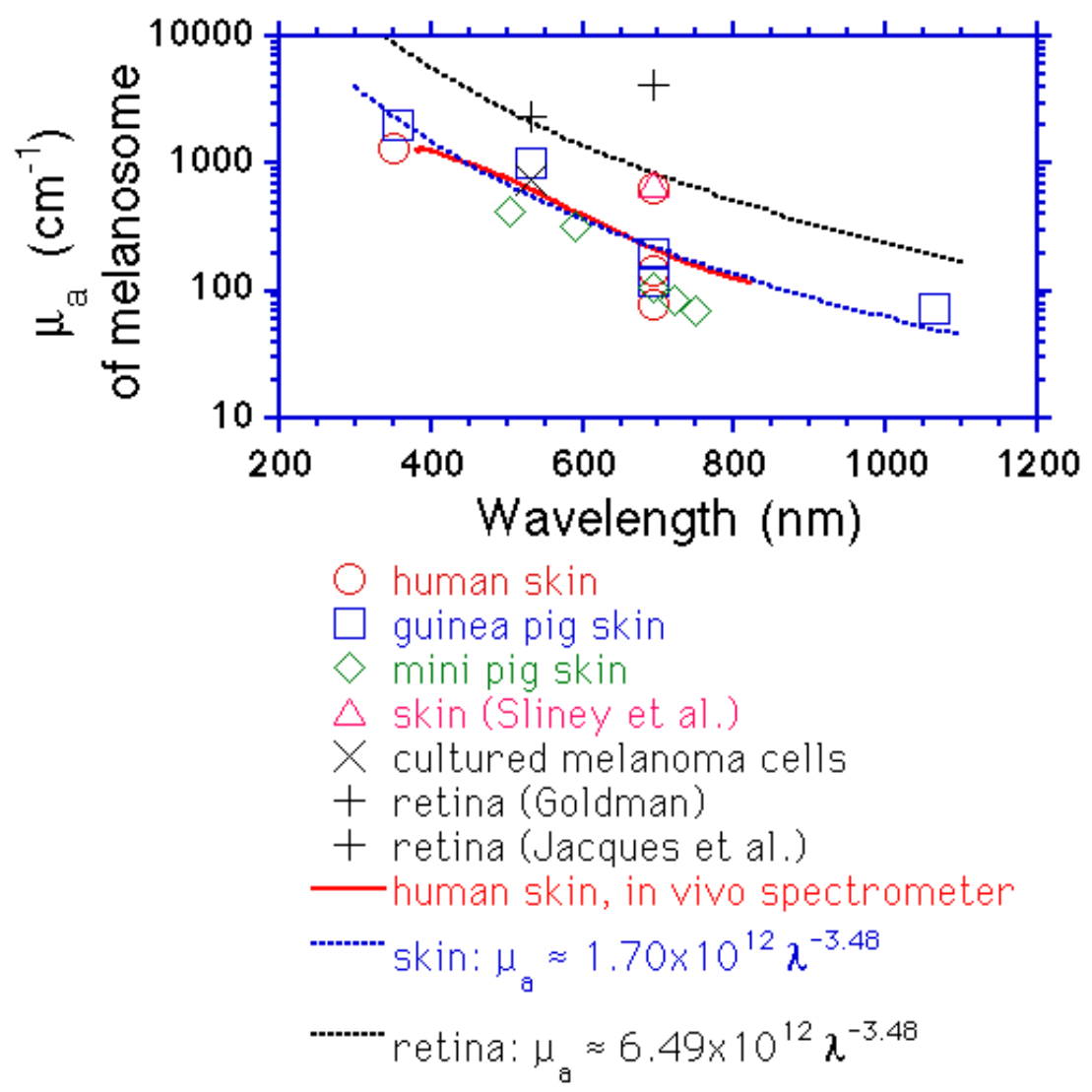

Figure 5.5 The absorption coefficient of melanosome.

To design the phantom solutions, a pilot study was performed to help understand the relationship between the measured signal intensities and phantom concentrations. Figure 5.6 shows the relationship between the OCT signal intensities and the concentration of Intralipid in the pilot phantom solutions. Higher concentration of Intralipid results in higher OCT signal intensities. Figure 5.7 shows the relationship between the PA signal intensities and the concentration of NIR dye in the pilot phantom solutions. Higher concentration of NIR dye results in higher PA signal intensities. For each phantom solution, the ratio of the processed OCT signal intensities to the PA signal intensities was calculated. The results are shown in Figure 5.8. We can find within a certain range of the ratio of the reduced scattering coefficient to the absorption coefficient, the OCT/PA ratios could be quite close to each other. 


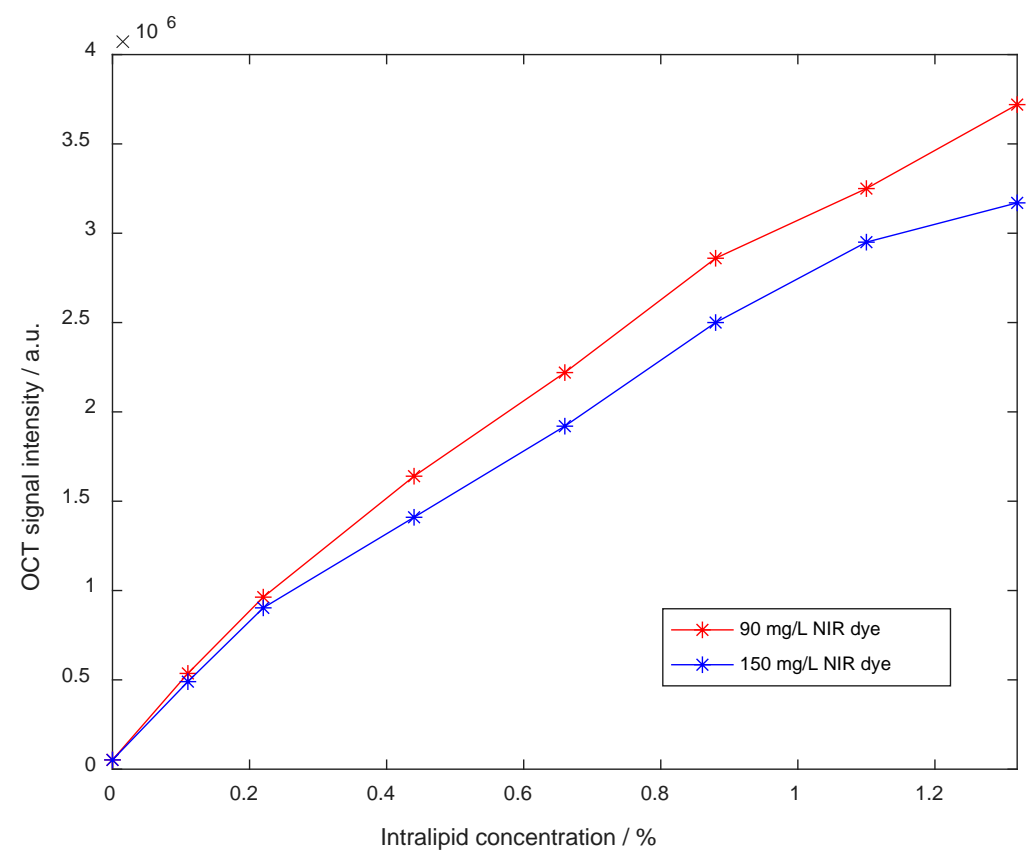

Figure 5.6 OCT signal intensities of pilot phantom solutions

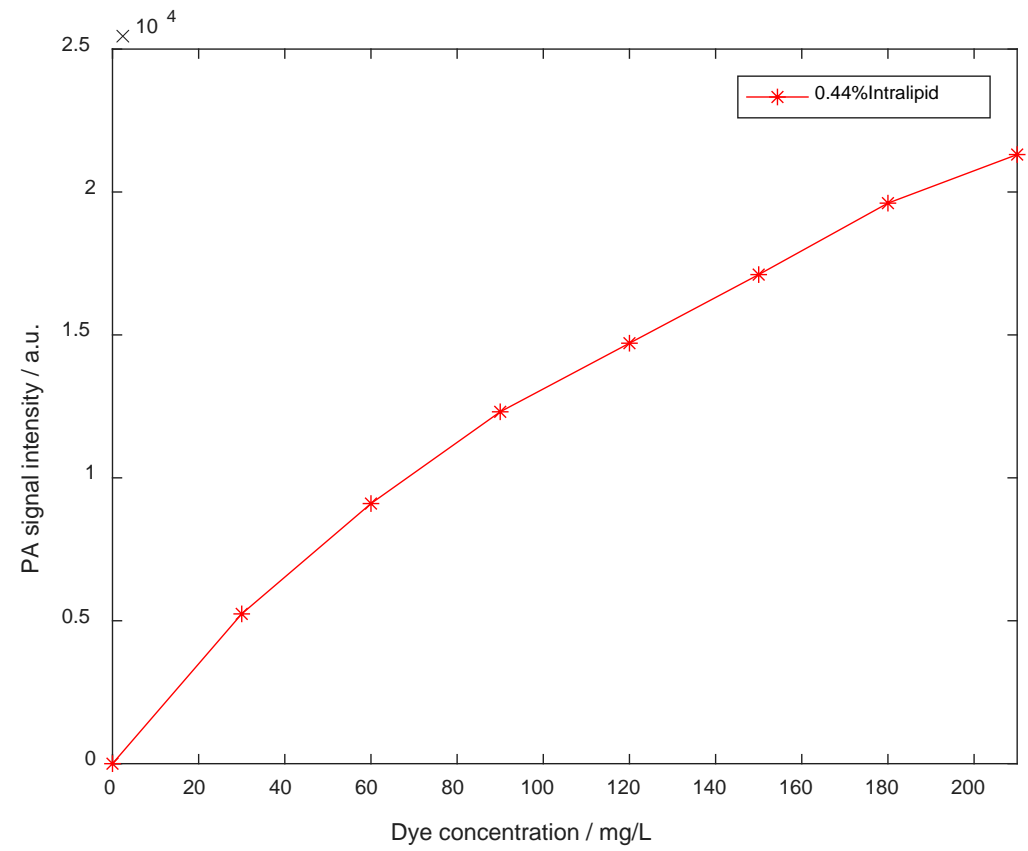

Figure 5.7 PA signal intensities of pilot phantom solutions 


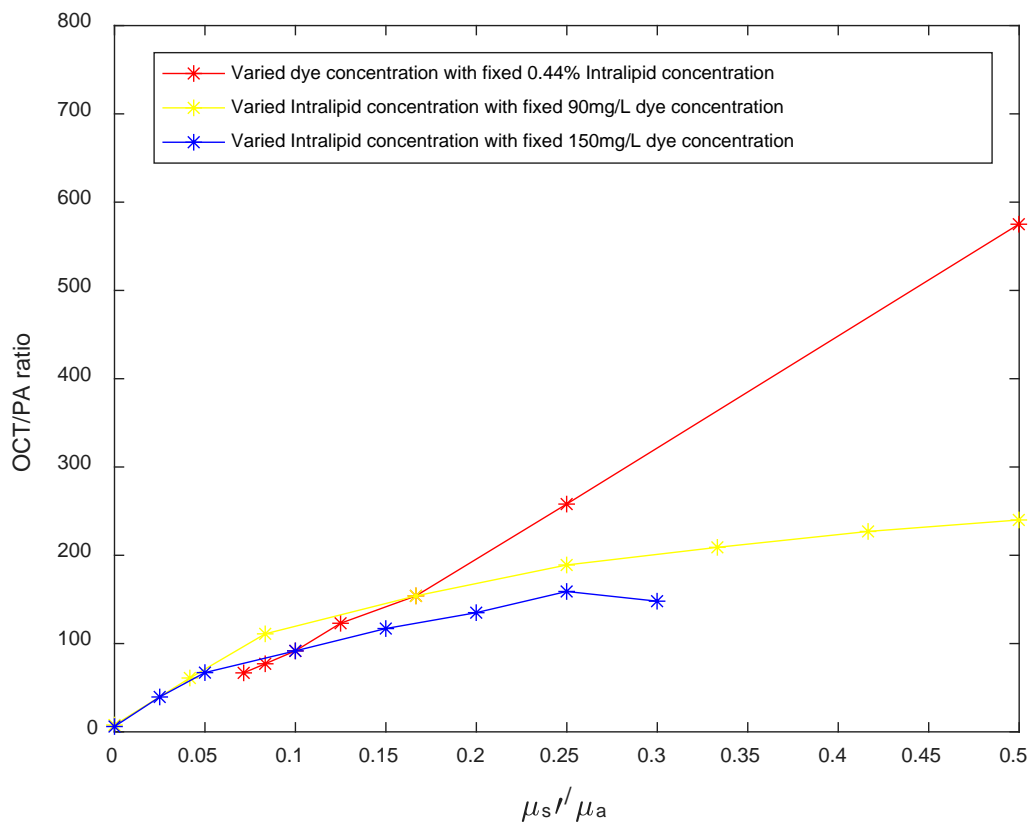

Figure 5.8 Ratio of OCT signal intensities to PA signal intensities of pilot phantom solutions

To link the phantom solutions to optical properties of biological tissues, some literature research was done to determine the concentration of phantom solutions. Hammer et al. measured the optical properties of bovine RPE, the absorption coefficient $\mu_{a}=380 \mathrm{~cm}^{-1}$ and the reduced scattering coefficient $\mu_{s}^{\prime}=256 \mathrm{~cm}^{-1}$.[134] Based on these studies and optical properties of RPE, the absorption coefficient of the phantom solution is designated to be $31.01 \mathrm{~cm}^{-1}$ to $620.2 \mathrm{~cm}^{-1}$ and the reduced scattering coefficient is designated to be from $18.6 \mathrm{~cm}^{-1}$ to $186.1 \mathrm{~cm}^{-1}$. To achieve a fixed ratio of the reduced scattering coefficient to the absorption coefficient from 0.3 to 0.6 , the concentration of Intralipid and NIR dye in the phantom solution is shown in Table 5.1. 
Table 5.1 Concentration of Intralipid and NIR dye in the phantom solution

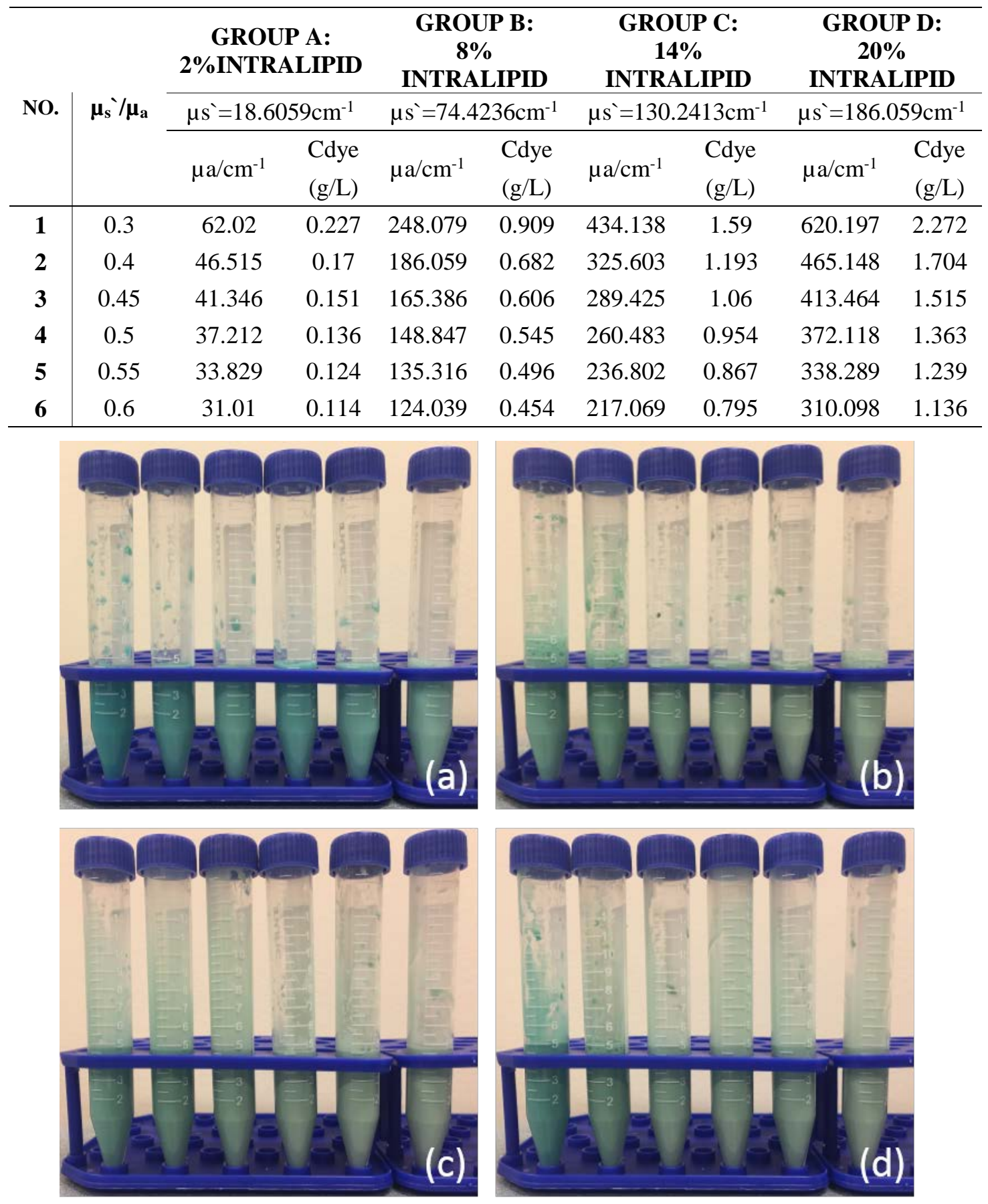

Figure 5.9 Photos of the phantom solutions prepared based on the mix ratio in Table 5.1. (a) Group A with 2\% Intralipid. (b) Group B with 8\% Intralipid. (c) Group C with 14\% Intralipid. (d) Group D with 20\% Intralipid. In each picture, the ratio of the reduced scattering coefficient to the absorption coefficient is $0.3,0.4,0.45,0.5,0.55$ and 0.6 from left to right. 
There are totally four groups of phantom solutions. For each group, a fixed concentration of Intralipid is mixed with varied concentrations of NIR dye to create phantom solutions with different ratios of the reduced scattering coefficient to the absorption coefficient, ranging from 0.3 to 0.6 . Since our hypothesis is related to optical properties, the ratios of the reduced scattering coefficient to the absorption coefficient is fixed as $0.3,0.4,0.45,0.5,0.55$ and 0.6. The concentrations of Intralipid from Group A to Group D are 2\%, 8\%, 14\% and 20\%, thus the concentration of NIR dye can be determined by the preset ratios and the optical scattering property of Intralipid. Figure 5.9 shows the photos of the phantom solutions prepared based on the mix ratios in Table 5.1. Figure 5.9(a)-(d) show the pictures of Group A to Group D with 2\%, $8 \%, 14 \%$ and 20\% Intralipid, respectively. In each picture, the ratios of the reduced scattering coefficient to the absorption coefficient are $0.3,0.4,0.45,0.5,0.55$ and 0.6 from left to right.

\subsubsection{Imaging System}

The experimental system is the one used for OC-PAM with intensity-modulated light source in Chapter 4, as shown in Figure 4.1. The phantom solution was injected into a polystyrene capillary tube (CTPS281-500-5, Paradigm Optics, Inc) by a syringe. The inside diameter of the polystyrene capillary tube is $281 \mu \mathrm{m}$ and the thickness of the wall is $109 \mu \mathrm{m}$. The tube was placed under the window of the water tank between the polyethylene membrane and a glass cube, and acoustically coupled with ultrasonic coupling gel. Both of the PAM and OCT images consist of $256 \times 20$ pixels, covering an area of $1.35 \times 0.2 \mathrm{~mm}^{2}$. 


\subsection{Results}

\subsubsection{OCT Signals}

Table 5.2 OCT signal intensities of phantom solutions

\begin{tabular}{|c|c|c|c|c|}
\hline$\mu s^{2} / \mu a$ & 2\%Intralipid & 8\% Intralipid & 14\% Intralipid & $20 \%$ Intralipid \\
\hline 0.3 & $9.4797 e+06$ & $1.9168 \mathrm{e}+07$ & $2.1362 \mathrm{e}+07$ & $2.4225 \mathrm{e}+07$ \\
\hline 0.4 & $1.0056 \mathrm{e}+07$ & $2.0999 \mathrm{e}+07$ & $2.4506 \mathrm{e}+07$ & $2.6592 \mathrm{e}+07$ \\
\hline 0.45 & $1.0564 \mathrm{e}+07$ & $2.2176 \mathrm{e}+07$ & $2.7408 \mathrm{e}+07$ & $2.9179 \mathrm{e}+07$ \\
\hline 0.5 & $1.0303 \mathrm{e}+07$ & $2.2853 \mathrm{e}+07$ & $2.6982 \mathrm{e}+07$ & $3.3028 \mathrm{e}+07$ \\
\hline 0.55 & $1.0342 \mathrm{e}+07$ & $2.2601 \mathrm{e}+07$ & $2.7675 \mathrm{e}+07$ & $3.1660 \mathrm{e}+07$ \\
\hline 0.6 & $1.0405 \mathrm{e}+07$ & $2.3148 \mathrm{e}+07$ & $2.7741 \mathrm{e}+07$ & $3.1434 \mathrm{e}+07$ \\
\hline
\end{tabular}

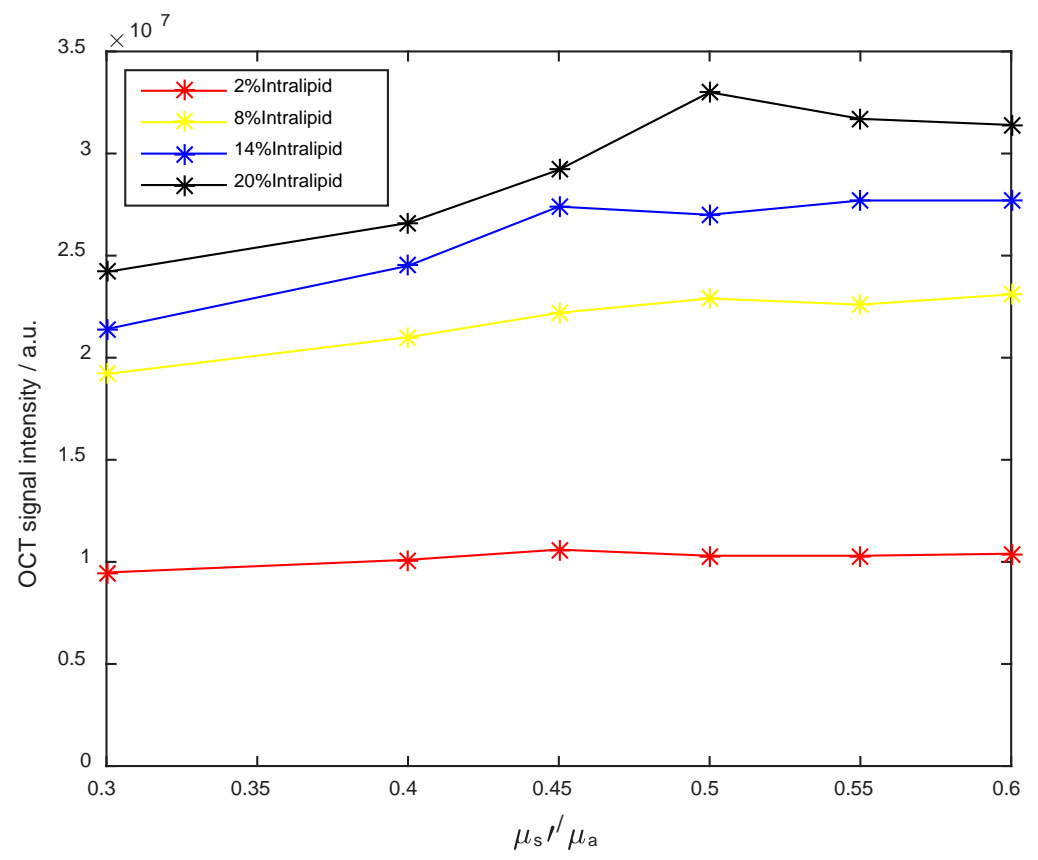

Figure 5.10 OCT signal intensities of phantom solutions

Based on Equation 5.1, the OCT signal intensity of phantom solution is presented by the square of the interpreted OCT signal in the time-domain. Table 5.2 shows the results of OCT signal 
intensities of different phantom solutions and the plot is shown in Figure 5.10. We can find that among those groups with different Intralipid concentrations, the whole group of phantom solutions with higher Intralipid concentration results in higher OCT signal intensities. The group with 2\% Intralipid reflects significantly lower OCT signal intensities because the concentration of Intralipid is only $1 / 4$ of $8 \%$ and $1 / 10$ of $20 \%$. Within the group of same Intralipid concentration, the OCT signal intensities increase smoothly with the increase of the $\mu_{s}^{\prime} / \mu_{a}$ ratio, which suggests that the existence of NIR dye also has minor effect on OCT signal intensities.

\subsubsection{PA Signals}

To evaluate the PA signal intensity, the measured PA signals were averaged and the average of background signal intensity was subtracted. Error! Not a valid bookmark self-reference. shows the results of PA signal intensities of different phantom solutions and the plot is shown in Figure 5.11. With higher absorption coefficient of the phantom solution, the processed PA signal intensities were increasing accordingly but nonlinearly with the existence of high scattering Intralipid.

Table 5.3 PA signal intensities of phantom solutions

\begin{tabular}{|c|c|c|c|c|}
\hline$\mu s ` / \mu a$ & 2\%Intralipid & 8\% Intralipid & 14\% Intralipid & $20 \%$ Intralipid \\
\hline 0.3 & $9.3783 e+03$ & $2.3098 \mathrm{e}+04$ & $2.8533 \mathrm{e}+04$ & $3.1480 \mathrm{e}+04$ \\
\hline 0.4 & $8.1944 \mathrm{e}+03$ & $2.1046 \mathrm{e}+04$ & $2.7173 e+04$ & $3.0623 \mathrm{e}+04$ \\
\hline 0.45 & $7.8560 \mathrm{e}+03$ & $2.1333 e+04$ & $2.7426 \mathrm{e}+04$ & $3.0183 e+04$ \\
\hline 0.5 & $6.9485 e+03$ & $2.0220 \mathrm{e}+04$ & $2.6216 \mathrm{e}+04$ & $2.8501 \mathrm{e}+04$ \\
\hline 0.55 & $6.9305 \mathrm{e}+03$ & $2.0452 \mathrm{e}+04$ & $2.6027 \mathrm{e}+04$ & $2.9142 \mathrm{e}+04$ \\
\hline 0.6 & $6.8175 \mathrm{e}+03$ & $2.0102 \mathrm{e}+04$ & $2.2618 \mathrm{e}+04$ & $2.6606 \mathrm{e}+04$ \\
\hline
\end{tabular}




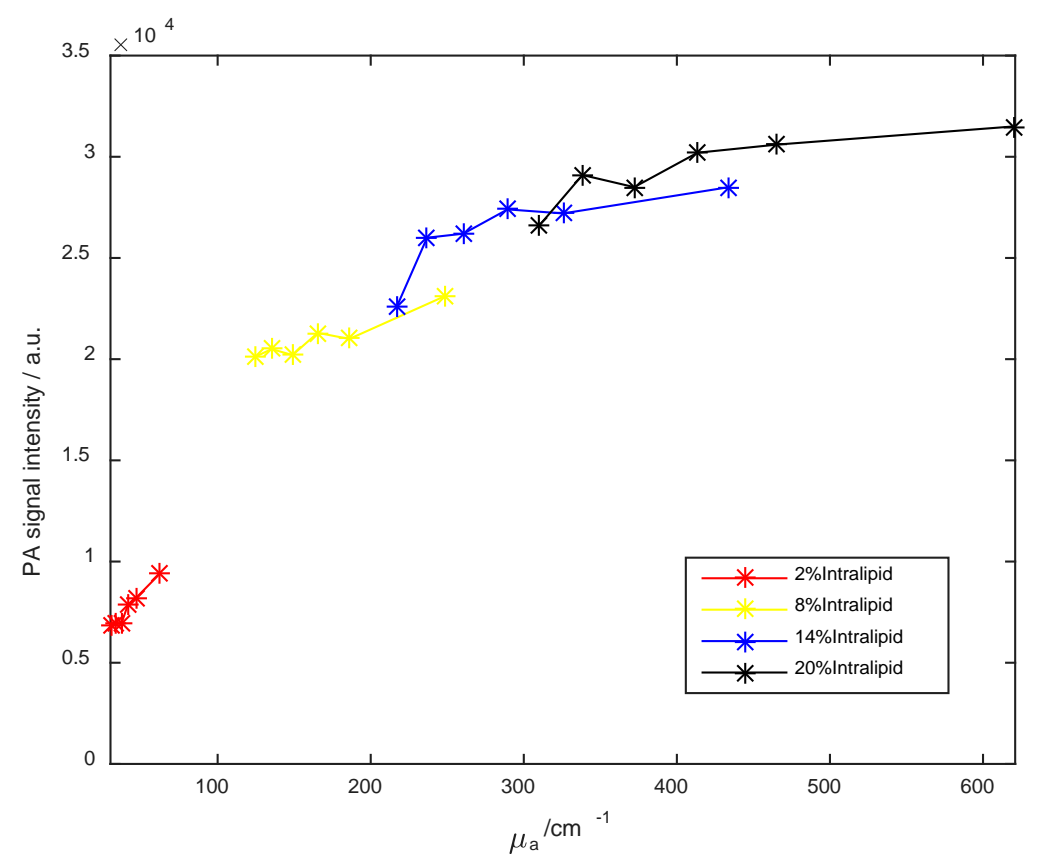

Figure 5.11 PA signal intensities of phantom solutions

\subsubsection{Relationship between OCT and PA Signals}

For each phantom solution, the ratio of the processed OCT signal intensities to the PA signal intensities was calculated. The results are shown in Table 5.4 and plotted in Figure 5.12. In Figure 5.12, we can find that for those groups with $8 \%, 14 \%$ and $20 \%$ Intralipid, the ratios of the OCT intensity to the PA intensity are very close when the ratios of the reduced scattering coefficient to the absorption coefficient are the same. However, for the group with $2 \%$ Intralipid, the line flies away from this trend, resulting in a significantly higher OCT/PA ratio. It could be caused by the error induced by the extremely small amount of NIR dye.

The average and standard deviation of the ratio of the OCT intensity to the PA intensity at each fixed ratio of the reduced scattering coefficient to the absorption coefficient are calculated and plotted in Figure 5.13. 
Table 5.4 Ratio of OCT signal intensities to PA signal intensities of phantom solutions

\begin{tabular}{ccccc}
\hline $\boldsymbol{\mu s}$ / $\boldsymbol{\mu a}$ & $\mathbf{2 \% \text { Intralipid }}$ & $\mathbf{8 \%}$ Intralipid & $\mathbf{1 4 \%}$ Intralipid & $\mathbf{2 0 \%}$ Intralipid \\
\hline 0.3 & $1.01 \mathrm{e}+03$ & $8.30 \mathrm{e}+02$ & $7.49 \mathrm{e}+02$ & $7.70 \mathrm{e}+02$ \\
0.4 & $1.23 \mathrm{e}+03$ & $9.98 \mathrm{e}+02$ & $9.02 \mathrm{e}+02$ & $8.68 \mathrm{e}+02$ \\
0.45 & $1.34 \mathrm{e}+03$ & $1.04 \mathrm{e}+03$ & $9.99 \mathrm{e}+02$ & $9.67 \mathrm{e}+02$ \\
0.5 & $1.48 \mathrm{e}+03$ & $1.13 \mathrm{e}+03$ & $1.03 \mathrm{e}+03$ & $1.16 \mathrm{e}+03$ \\
0.55 & $1.49 \mathrm{e}+03$ & $1.11 \mathrm{e}+03$ & $1.06 \mathrm{e}+03$ & $1.09 \mathrm{e}+03$ \\
0.6 & $1.53 \mathrm{e}+03$ & $1.15 \mathrm{e}+03$ & $1.23 \mathrm{e}+03$ & $1.18 \mathrm{e}+03$ \\
\hline
\end{tabular}

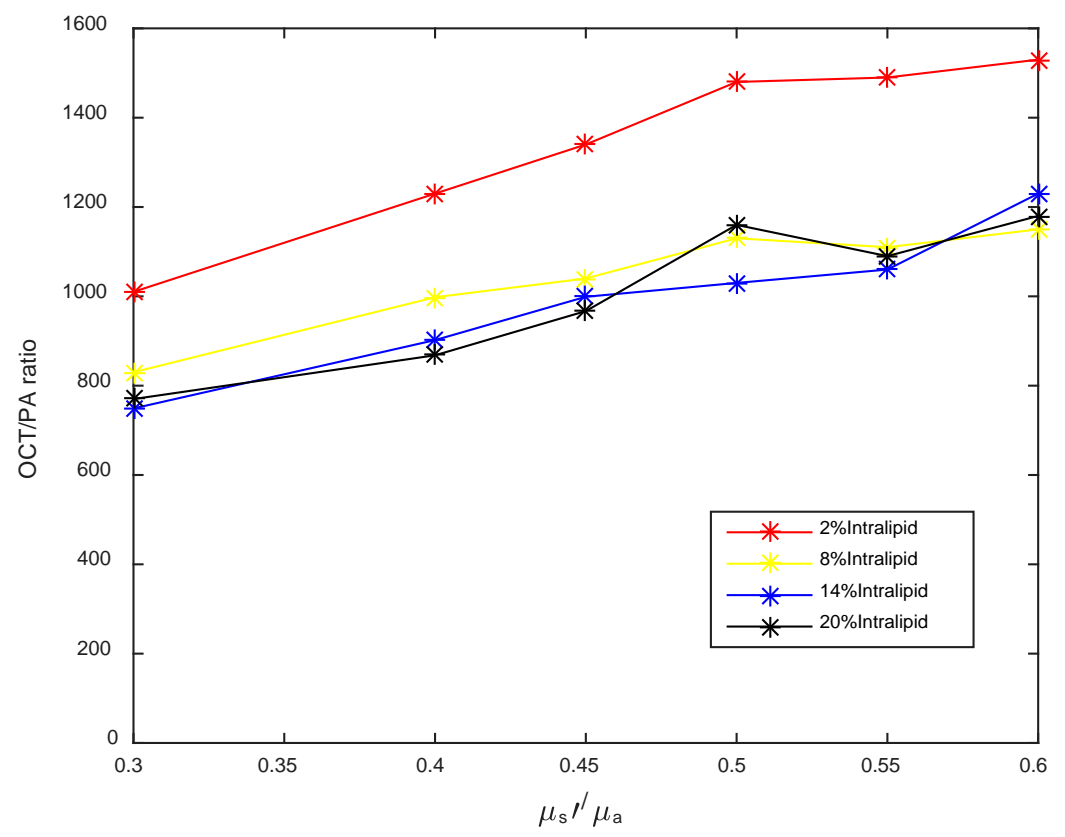

Figure 5.12 Ratio of OCT signal intensities to PA signal intensities of phantom solutions 


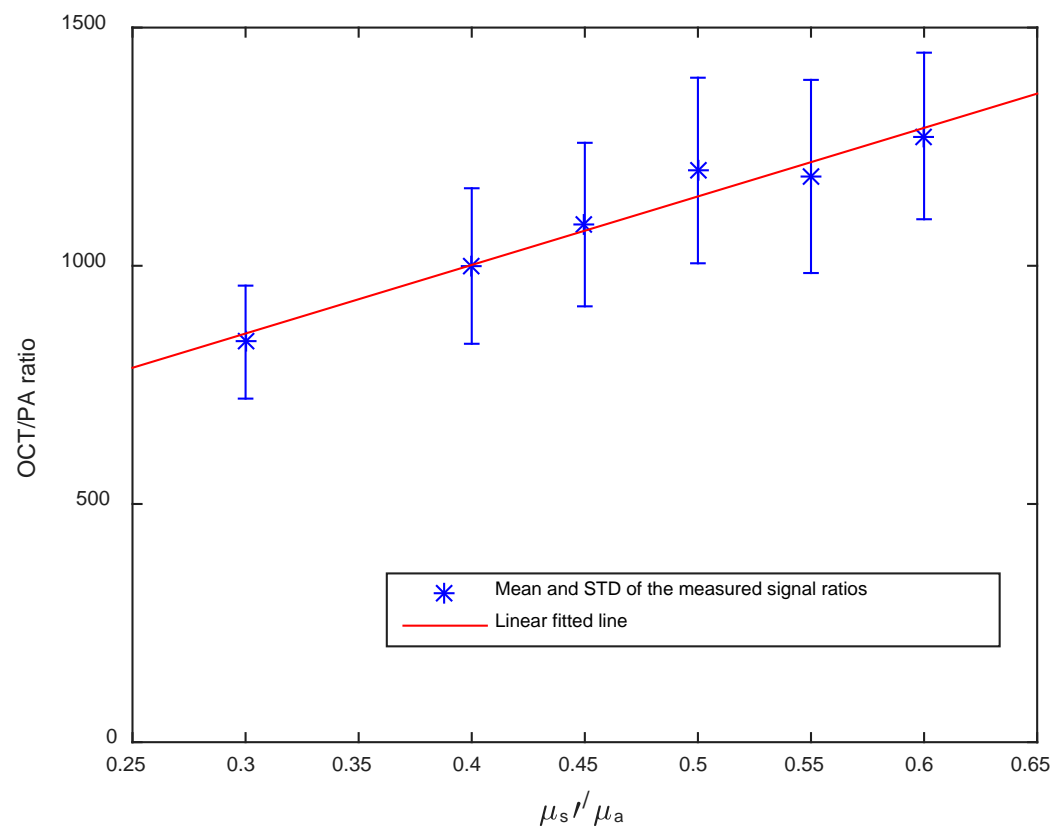

Figure 5.13 Mean and standard deviation of the ratio of OCT signal intensities to PA signal intensities of phantom solutions

Based on the assumption discussed in Chapter 5.2 and the trend of the plotted data, a linear model was used to fit the averaged data. The fitting result is expressed as:

$$
R_{O C T / P A}=1439 * R_{\mu_{s}^{\prime} / \mu_{a}}+426.2,
$$

where $R_{O C T / P A}$ is the ratio of processed OCT signal intensities to PA signal intensities of phantom solutions, $R_{\mu_{s}^{\prime} / \mu_{a}}$ is the ratio of the reduced scattering coefficient to the absorption coefficient of phantom solutions. The $\mathrm{R}^{2}$ for this fitting is 0.9629 , which shows a good fit of the data.

Thus, by transforming Equation 5.13, the ratio related to the optical properties of the imaged phantom solutions by OC-PAM system can be expressed as:

$$
R_{\mu_{s}^{\prime} / \mu_{a}}=0.000669 * R_{O C T / P A}-0.268,
$$

This result may indicate the relationship between optical properties and images acquired by the proposed NIR OC-PAM system. 


\subsection{Discussion}

In this phantom study of the NIR OC-PAM system, the imaging model was simplified as a monolayer of scatterers and absorbers when discussing the theoretical model, thus a linear relationship was interpreted from the measured OCT and PA signals. However, the real eye has a more complicated structure. Therefore, to study the optical properties of RPE, a more comprehensive model with characteristics of all layers before and after RPE is preferred and will be built and discussed in the future.

Another concern about this phantom study is the accuracy about phantom preparation. Even though the measurement was repeated more than once on these phantom solutions, the accuracy of the phantom solution should also be taken into consideration and ideally more than one phantom solution should be prepared for each mixed concentration. However, limited by the phantom materials, this concern haven’t been eliminated by preparing more groups of phantom solutions and creating more data to perform curve fitting.

More work can be done regarding the calibration and normalization of the measured signals on different subjects and under different circumstances. To build more reliable evidence to evaluate the existence of melanin and lipofuscin specifically, thus to benefit the diagnosis and research of AMD, the normal model and diseased model are both necessary to be imaged by this NIR OCPAM system.

In general, to be statistically reliable and valid, future work will be focused on acquiring more data points to create the plot representing the relationship between the signal intensities and optical properties so as to calculate more accurate coefficients for the fitted line. 


\subsection{Conclusion}

In conclusion, a phantom study was conducted with the NIR OC-PAM system in this chapter. The relationship between the image intensities of OCT and PAM and the relationship between the optical scattering property and optical absorption property was modeled and fitted with the data acquired from the phantom solutions. The assumption for this phantom study that for a certain phantom with a fixed ratio of the scattering and absorption coefficients, the interpreted signal strength of OCT and photoacoustic from the acquired images would be accordingly related has been proven by a simplified modeling. This study would potentially enhance the practical application of the NIR OC-PAM system for studying optical properties of biological tissues. 


\section{CHAPTER 6. SUMMARY}

Optical coherence tomography (OCT) and Photoacoustic microscopy (PAM) are two noninvasive high-resolution three-dimensional biomedical imaging modalities based on different contrast mechanisms. OCT detects the light backscattered from a biological sample either in the time or spectral domain using an interferometer to form an image. PAM is sensitive to optical absorption by detecting the light induced acoustic waves to form an image. Due to their complementary contrast mechanisms OCT and PAM are suitable for being combined to achieve multimodal imaging.

The goals of the dissertation were:

1. To develop optical coherence photoacoustic microscopy (OC-PAM) for in vivo multimodal retinal imaging with a pulsed broadband NIR light source.

2. To develop OC-PAM for in vivo multimodal imaging with an intensity-modulated continuous-wave light source in the NIR.

3. In vivo application of NIR OC-PAM on small animals.

4. To conduct a phantom study and extract quantitative information of the optical properties from OC-PAM.

In the first part of this dissertation, a spectral-domain optical coherence tomography (SD-OCT) system was built for in vivo imaging of rodent retina to evaluate the effect of powerless contact lens on improving the quality of optical coherence tomography imaging of rodent retina. A commercial powerless contact lens for rat eye was tested in the experiments. The fundus images generated from the measured 3D OCT datasets with contact lens showed sharper retinal blood vessels than those without contact lens. The contrast of the retinal blood vessels was also significantly enhanced in the OCT fundus images with contact lens. As high as $10 \mathrm{~dB}$ improvements in SNR was observed 
for OCT images with contact lens compared to the images of the same retinal area without contact lens. We have demonstrated for the first time that the use of powerless contact lens on rat eye can significantly improve OCT image quality of rodent retina, which is a benefit in addition to preventing cataract formation. We believe the improvement in image quality is the result of partial compensation of the optical aberrations of the rodent eye by the contact lens.

As a follow-up study of this topic, another OCT system was built to image the anterior segment of rat's eye in order to observe the condition of cornea. The results in this follow-up study show the structure of anterior segment of rat eye, and cornea defects were observed on most of the rats' eye. These uneven surfaces of cornea may induce severe aberrations to the eye when performing optical imaging such as OCT on the eye. After applying contact lens, the surface of eye became smooth and even, which improves the optics system of anterior segment of rat eye. This supplement study is a support of our previous hypothesis that the improvement of OCT image quality by applying contact lens is a result of compensation of the optical aberration caused by the defects of rat cornea.

In the second part of this dissertation, an optical coherence photoacoustic microscopy (OCPAM) system, which can accomplish optical coherence tomography (OCT) and photoacoustic microscopy (PAM) simultaneously by using a single pulsed broadband light source, was developed. With a center wavelength of $800 \mathrm{~nm}$ and a bandwidth of $30 \mathrm{~nm}$ the system is suitable for imaging the retina. Generated from the same group of photons, the OCT and PAM images are intrinsically registered in the lateral directions. To test the capabilities of the system on multimodal ophthalmic imaging, we imaged the retina of pigmented rats. The OCT images showed the retinal structures with quality similar to conventional OCT, while the PAM images revealed the distribution of absorbers in the retina. Since the absorption of hemoglobin is relatively weak at 
around $800 \mathrm{~nm}$, the NIR PAM signals are generated mainly from melanin in the posterior segment of the eye, thus provide melanin-specific imaging of the retina.

Although no damage to the rat retina was observed after at least ten times of imaging experiments in a period of 6 months, the fact is that the pulse energy of $\sim 400 \mathrm{~nJ}$ in this experiment is higher than the MPE according to the ANSI laser safety standard. We believe the pulse energy can be lowered when a transducer with better sensitivity is available. Limited by the relatively slow PRR of the light source, the imaging speed is not as high as conventional spectral-domain OCT. Increasing the PRR can increase the imaging speed. However, it will also increase the average power of the light source, which may cause laser safety issues in practical applications. In all situations an ultrasonic transducer with high sensitivity is a key for reducing the laser pulse energy, thus makes it safe for eye imaging.

In the third part of this dissertation, an optical coherence photoacoustic microscopy (OC-PAM) system using an intensity-modulated continuous-wave (CW) superluminescent diode with a center wavelength of $840 \mathrm{~nm}$ was developed. The system can also accomplish optical coherence tomography (OCT) and photoacoustic microscopy (PAM) simultaneously. Compared to the system with a pulsed light source in the second part of this dissertation, this system is able to achieve OCT imaging with quality as high as conventional spectral-domain OCT. Since both of the OCT and PAM images are generated from the same group of photons, they are intrinsically registered in the lateral directions. The system was tested for multimodal imaging the vasculature of mouse ear in vivo by using Gold Nanorods (GNRs) as contrast agent for PAM, as well as excised porcine eyes ex vivo. The OCT and PAM images showed complimentary information of the sample.

Since the imaging speed of the system is mainly limited by the mechanical scanning speed of the translational stages and the integration time of the lock-in amplifier used for photoacoustic 
signal processing, a total of more than 30 minutes is needed to acquire the whole PAM image. The slow imaging speed may limit the system for in vivo applications such as retinal imaging. Improved scanning mechanisms such as optical scanning with galvanometer and increased modulation frequency of the light source may help improve the imaging speed.

To further compare the OCT image quality fairly, the pulsed light source used in the second part and the intensity-modulated broadband SLD light source used in this part were combined by a flip mirror and coupled into the source arm of fiber-based OCT system. Compared with the OCT image acquired with pulse broadband light source, the one acquired with intensity-modulated cw light source shows stronger signal intensity and better contrast to the background. Furthermore, the layered structures in retina are better resolved in the OCT image acquired with intensity-modulated cw light source. However, the PAM image quality in this topic is not very good due to limitation of the ultrasonic transducer sensitivity. As a result, contrast agent needs to be used for vasculature imaging. As reported by other group, a possible $20 \mathrm{~dB}$ of magnitude improvement can be expected when a resonant transducer is used[111]. In our system setup, the glass cube at the back of the sample also improved (roughly doubled) the photoacoustic signal by reflecting the ultrasound transmitted through the sample. However, this OC-PAM system is configured in the transmission mode due to limited availability of the ultrasonic transducer. We believe that the availability of better transducers could allow the system to be configured in the reflection mode, which is more suitable for in vivo imaging.

In the final part of this dissertation, a phantom study was conducted with the NIR OC-PAM system. The relationship between the image intensities of OCT and PAM and the relationship between the optical scattering property and optical absorption property was modeled and fitted with the data acquired from the phantom solutions. The assumption for this phantom study that for a 
certain phantom with fixed ratio of the scattering and absorption coefficient, the interpreted signal strength of OCT and photoacoustic from the acquired images would be accordingly related has been proved by a simplified modeling. This study would potentially enhance the practical application of the NIR OC-PAM system for studying optical properties of biological tissues.

In this phantom study of the NIR OC-PAM system, the imaging model was simplified as a monolayer of scatterers and absorbers when discussing the theoretical model, thus a linear relationship was interpreted from the measured OCT and PA signals. However, the real eye has a more complicated structure. Therefore, to study the optical properties of RPE, a more comprehensive model with characteristics of all layers before and after RPE is preferred and will be built and discussed in the future. In general, to be statistically reliable and valid, future work will be focused on acquiring more data points to create the plot representing the relationship between the signal intensities and optical properties so as to calculate more accurate coefficients for the fitted line.

In conclusion, there are several areas of potential improvement and further development:

- First of all, the sensitivity of the ultrasonic transducer is the main limitation of PAM image quality. Due to the limited availability of the ultrasonic transducer, the quality of our current PAM imaging modality hasn’t reached the best of the current photoacoustic imaging technique and the reflection mode of photoacoustic detection would be more suitable for in vivo imaging than the transmission mode. Therefore, if an ultrasonic transducer with better sensitivity is employed, the practical application of the proposed NIR OC-PAM system will be enhanced.

- $\quad$ Second, more work can be done regarding the phantom study to build more reliable evidence to evaluate the existence of melanin and lipofuscin specifically. For example, 
the measured signals on different subjects and under different circumstances can be calibrated, a more comprehensive model with the optical properties of all layers before and after RPE can be built, and the normal model and diseased model should be compared to benefit the diagnosis and research of AMD.

- Third, the current NIR OC-PAM system is a table-top experimental prototype, which lacks flexibilities for clinical application. Thus, if a hand-held detection head of NIR OC-PAM is built, the proposed system will be able to achieve maximum flexibility. 


\section{LIST OF REFERENCES}

[1] Huang D, Swanson E A, Lin C P, Schuman J S, Stinson W G, Chang W, Hee M R, Flotte T, Gregory K and Puliafito C A 1991 Optical coherence tomography Science 254 1178-81

[2] Wojtkowski M, Srinivasan V, Ko T, Fujimoto J, Kowalczyk A and Duker J 2004 Ultrahigh-resolution, high-speed, Fourier domain optical coherence tomography and methods for dispersion compensation Optics express 12 2404-22

[3] Swanson E A, Izatt J, Lin C, Fujimoto J, Schuman J, Hee M, Huang D and Puliafito C 1993 In vivo retinal imaging by optical coherence tomography Optics letters 18 1864-6

[4] Hee M R, Puliafito C A, Wong C, Duker J S, Reichel E, Rutledge B, Schuman J S, Swanson E A and Fujimoto J G 1995 Quantitative assessment of macular edema with optical coherence tomography Archives of ophthalmology 113 1019-29

[5] Hee M R, Baumal C R, Puliafito C A, Duker J S, Reichel E, Wilkins J R, Coker J G, Schuman J S, Swanson E A and Fujimoto J G 1996 Optical coherence tomography of age-related macular degeneration and choroidal neovascularization Ophthalmology 103 1260-70

[6] Uchino E, Uemura A and Ohba N 2001 Initial stages of posterior vitreous detachment in healthy eyes of older persons evaluated by optical coherence tomography Archives of Ophthalmology 119 1475-9

[7] Altaweel M and Ip M 2003 Macular hole: improved understanding of pathogenesis, staging, and management based on optical coherence tomography. In: Seminars in ophthalmology: Taylor \& Francis) pp 58-66

[8] Medeiros F A, Zangwill L M, Bowd C, Vessani R M, Susanna R and Weinreb R N 2005 Evaluation of retinal nerve fiber layer, optic nerve head, and macular thickness measurements for glaucoma detection using optical coherence tomography American journal of ophthalmology 139 44-55

[9] Pircher M, Götzinger E, Leitgeb R, Fercher A and Hitzenberger C 2003 Measurement and imaging of water concentration in human cornea with differential absorption optical coherence tomography Optics Express 11 2190-7

[10] Costa R A, Scapucin L, Moraes N S, Calucci D, Melo L A, Cardillo J A and Farah M E 2002 Indocyanine green-mediated photothrombosis as a new technique of treatment for persistent central serous chorioretinopathy Current eye research 25 287-97 
[11] Campochiaro P A 2004 Reduction of diabetic macular edema by oral administration of the kinase inhibitor PKC412 Investigative ophthalmology \& visual science 45 922-31

[12] Michels S, Rosenfeld P J, Puliafito C A, Marcus E N and Venkatraman A S 2005 Systemic bevacizumab (Avastin) therapy for neovascular age-related macular degeneration: twelve-week results of an uncontrolled open-label clinical study Ophthalmology 112 1035-47. e9

[13] Gladkova N D, Petrova G, Nikulin N, Radenska - Lopovok S, Snopova L, Chumakov Y P, Nasonova V, Gelikonov V, Gelikonov G and Kuranov R 2000 In vivo optical coherence tomography imaging of human skin: norm and pathology Skin Research and Technology 6 6-16

[14] Welzel J 2001 Optical coherence tomography in dermatology: a review Skin Research and Technology 7 1-9

[15] Brezinski M E, Tearney G J, Bouma B E, Izatt J A, Hee M R, Swanson E A, Southern J F and Fujimoto J G 1996 Optical coherence tomography for optical biopsy properties and demonstration of vascular pathology Circulation 93 1206-13

[16] Brezinski M E, Tearney G J, Bouma B E, Boppart S A, Hee M R, Swanson E A, Southern J F and Fujimoto J G 1996 Imaging of coronary artery microstructure (in vitro) with optical coherence tomography The American journal of cardiology 77 92-3

[17] Fujimoto J, Boppart S A, Tearney G, Bouma B, Pitris C and Brezinski M 1999 High resolution in vivo intra-arterial imaging with optical coherence tomography Heart 82 128-33

[18] Gupta M, Rollins A M, Izatt J A and Efimov I R 2002 Imaging of the atrioventricular node using optical coherence tomography Journal of cardiovascular electrophysiology 13 95-

[19] Jang I-K, Tearney G J, MacNeill B, Takano M, Moselewski F, Iftima N, Shishkov M, Houser S, Aretz H T and Halpern E F 2005 In vivo characterization of coronary atherosclerotic plaque by use of optical coherence tomography Circulation 111 1551-5

[20] Tearney G J, Yabushita H, Houser S L, Aretz H T, Jang I-K, Schlendorf K H, Kauffman C R, Shishkov M, Halpern E F and Bouma B E 2003 Quantification of macrophage content in atherosclerotic plaques by optical coherence tomography Circulation 107 113-9

[21] Izatt J A, Kulkarni M D, Wang H-W, Kobayashi K and Sivak M V 1996 Optical coherence tomography and microscopy in gastrointestinal tissues IEEE Journal of Selected topics in quantum electronics 2 1017-28 
[22] Brand S, Poneros J, Bouma B, Tearney G, Compton C and Nishioka N 2000 Optical coherence tomography in the gastrointestinal tract Endoscopy 32 796-803

[23] Jäckle S, Gladkova N, Feldchtein F, Terentieva A, Brand B, Gelikonov G, Gelikonov V, Sergeev A, Fritscher-Ravens A and Freund J 2000 In vivo endoscopic optical coherence tomography of the human gastrointestinal tract-toward optical biopsy Endoscopy 32 743-9

[24] Sivak M V, Kobayashi K, Izatt J A, Rollins A M, Ung-Runyawee R, Chak A, Wong R C, Isenberg G A and Willis J 2000 High-resolution endoscopic imaging of the GI tract using optical coherence tomography Gastrointestinal endoscopy 51 474-9

[25] Boppart S A, Luo W, Marks D L and Singletary K W 2004 Optical coherence tomography: feasibility for basic research and image-guided surgery of breast cancer Breast cancer research and treatment 84 85-97

[26] Zysk A M and Boppart S A 2006 Computational methods for analysis of human breast tumor tissue in optical coherence tomography images Journal of biomedical optics 11 054015--7

[27] Drexler W, Stamper D, Jesser C, Li X, Pitris C, Saunders K, Martin S, Lodge M B, Fujimoto J G and Brezinski M E 2001 Correlation of collagen organization with polarization sensitive imaging of in vitro cartilage: implications for osteoarthritis The Journal of rheumatology 28 1311-8

[28] Li X, Martin S, Pitris C, Ghanta R, Stamper D L, Harman M, Fujimoto J G and Brezinski M E 2005 High-resolution optical coherence tomographic imaging of osteoarthritic cartilage during open knee surgery Arthritis Res Ther 71

[29] Armstrong J, Leigh M, Walton I, Zvyagin A, Alexandrov S, Schwer S, Sampson D, Hillman D and Eastwood P 2003 In vivo size and shape measurement of the human upper airway using endoscopic longrange optical coherence tomography Optics express 11 1817-26

[30] Freitas A, Zezell D, Vieira Jr N, Ribeiro A and Gomes A 2006 Imaging carious human dental tissue with optical coherence tomography Journal of Applied Physics $\mathbf{9 9}$ 024906

[31] Jiao S 2004 Polarization-sensitive Mueller-matrix optical coherence tomography. Texas A\&M University)

[32] Schmitt J M 1999 Optical coherence tomography (OCT): a review IEEE Journal of selected topics in quantum electronics 5 1205-15

[33] Chen T C, Cense B, Pierce M C, Nassif N, Park B H, Yun S H, White B R, Bouma B E, Tearney G J and de Boer J F 2005 Spectral domain optical coherence 
tomography: ultra-high speed, ultra-high resolution ophthalmic imaging Archives of Ophthalmology 123 1715-20

[34] Nassif N, Cense B, Park B, Pierce M, Yun S, Bouma B, Tearney G, Chen T and de Boer J 2004 In vivo high-resolution video-rate spectral-domain optical coherence tomography of the human retina and optic nerve Optics express 12 367-76

[35] Jiao S, Knighton R, Huang X, Gregori G and Puliafito C 2005 Simultaneous acquisition of sectional and fundus ophthalmic images with spectral-domain optical coherence tomography Optics Express 13 444-52

[36] Goodman J 1985 Statistical Optics John Wiley \& Sons New York

[37] Zhang H F, Maslov K, Stoica G and Wang L V 2006 Functional photoacoustic microscopy for high-resolution and noninvasive in vivo imaging Nature biotechnology 24 848-51

[38] Maslov K, Zhang H F, Hu S and Wang L V 2008 Optical-resolution photoacoustic microscopy for in vivo imaging of single capillaries Optics letters 33 92931

[39] Jiao S, Jiang M, Hu J, Fawzi A, Zhou Q, Shung K K, Puliafito C A and Zhang H F 2010 Photoacoustic ophthalmoscopy for in vivo retinal imaging Optics express 18 3967-72

[40] Zhang H F, Maslov K and Wang L V 2007 In vivo imaging of subcutaneous structures using functional photoacoustic microscopy Nature protocols 2 797-804

[41] Hu S and Wang L V 2010 Photoacoustic imaging and characterization of the microvasculature Journal of biomedical optics 15 011101--15

[42] Wang L V 2009 Multiscale photoacoustic microscopy and computed tomography Nature photonics 3 503-9

[43] Bell A G 1880 On the production and reproduction of sound by light American Journal of Science 305-24

[44] Maugh T H 1975 Photoacoustic spectroscopy: New uses for an old technique Science 188 38-9

[45] Kruger R A, Liu P and Appledorn C R 1995 Photoacoustic ultrasound (PAUS) reconstruction tomography Medical physics 22 1605-9

[46] Li C and Wang L V 2009 Photoacoustic tomography and sensing in biomedicine Physics in medicine and biology 54 R59 
[47] Wang X, Pang Y, Ku G, Xie X, Stoica G and Wang L V 2003 Noninvasive laserinduced photoacoustic tomography for structural and functional in vivo imaging of the brain Nature biotechnology 21 803-6

[48] Zhang E Z, Povazay B, Laufer J, Alex A, Hofer B, Pedley B, Glittenberg C, Treeby B, Cox B and Beard P 2011 Multimodal photoacoustic and optical coherence tomography scanner using an all optical detection scheme for 3D morphological skin imaging Biomedical optics express 2 2202-15

[49] Favazza C P, Jassim O, Cornelius L A and Wang L V 2011 In vivo photoacoustic microscopy of human cutaneous microvasculature and a nevus Journal of biomedical optics 16 016015--6

[50] Jansen K, Van Der Steen A F, van Beusekom H M, Oosterhuis J W and van Soest G 2011 Intravascular photoacoustic imaging of human coronary atherosclerosis Optics letters 36 597-9

[51] Wang B, Yantsen E, Larson T, Karpiouk A B, Sethuraman S, Su J L, Sokolov K and Emelianov S Y 2008 Plasmonic intravascular photoacoustic imaging for detection of macrophages in atherosclerotic plaques Nano Letters 9 2212-7

[52] Yang J-M, Maslov K, Yang H-C, Zhou Q, Shung K K and Wang L V 2009 Photoacoustic endoscopy Optics letters 34 1591-3

[53] Yang J-M, Favazza C, Chen R, Yao J, Cai X, Maslov K, Zhou Q, Shung K K and Wang L V 2012 Toward dual-wavelength functional photoacoustic endoscopy: laser and peripheral optical systems development. In: SPIE BiOS: International Society for Optics and Photonics) pp 822316--7

[54] Erpelding T N, Kim C, Pramanik M, Jankovic L, Maslov K, Guo Z, Margenthaler J A, Pashley M D and Wang L V 2010 Sentinel lymph nodes in the rat: noninvasive photoacoustic and US imaging with a clinical US system 1 Radiology 256 102-10

[55] Kim C, Cho E C, Chen J, Song K H, Au L, Favazza C, Zhang Q, Cobley C M, Gao F and Xia Y 2010 In vivo molecular photoacoustic tomography of melanomas targeted by bioconjugated gold nanocages ACS nano 4 4559-64

[56] Hu S and Wang L V 2010 Neurovascular photoacoustic tomography Front. Neuroenergetics 2

[57] Hu S, Yan P, Maslov K, Lee J-M and Wang L V 2009 Intravital imaging of amyloid plaques in a transgenic mouse model using optical-resolution photoacoustic microscopy Optics letters 34 3899-901

[58] Hu S, Rao B, Maslov K and Wang L V 2010 Label-free photoacoustic ophthalmic angiography Optics letters 35 1-3 
[59] Zemp R J, Bitton R, Li M-L, Shung K K, Stoica G and Wang L V 2007 Photoacoustic imaging of the microvasculature with a high-frequency ultrasound array transducer Journal of biomedical optics 12 010501--3

[60] Brecht H-P, Su R, Fronheiser M, Ermilov S A, Conjusteau A and Oraevsky A A 2009 Whole-body three-dimensional optoacoustic tomography system for small animals Journal of biomedical optics 14 064007--8

[61] Li C, Aguirre A, Gamelin J, Maurudis A, Zhu Q and Wang L V 2010 Real-time photoacoustic tomography of cortical hemodynamics in small animals Journal of biomedical optics 15 010509--3

[62] Maslov K, Stoica G and Wang L V 2005 In vivo dark-field reflection-mode photoacoustic microscopy Optics letters 30 625-7

[63] Zhang H F, Puliafito C A and Jiao S 2011 Photoacoustic ophthalmoscopy for in vivo retinal imaging: current status and prospects Ophthalmic Surgery, Lasers and Imaging Retina 42 S106-S15

[64] Song K H and Wang L V 2007 Deep reflection-mode photoacoustic imaging of biological tissue Journal of biomedical optics 12 060503--3

[65] Ma T, Zhang X, Chiu C T, Chen R, Shung K K, Zhou Q and Jiao S 2014 Systematic study of high-frequency ultrasonic transducer design for laser-scanning photoacoustic ophthalmoscopy Journal of biomedical optics 19 016015-

[66] Guittet C, Ossant F, Vaillant L and Berson M 1999 In vivo high-frequency ultrasonic characterization of human dermis IEEE transactions on biomedical engineering 46 740-6

[67] Jiao S, Xie Z, Zhang H F and Puliafito C A 2009 Simultaneous multimodal imaging with integrated photoacoustic microscopy and optical coherence tomography Optics letters 34 2961-3

[68] Diebold G, Sun T and Khan M 1991 Photoacoustic monopole radiation in one, two, and three dimensions Physical review letters 673384

[69] Deán-Ben X L, Razansky D and Ntziachristos V 2011 Correction for acoustic attenuation effects in optoacoustic tomographic reconstructions. In: European Conference on Biomedical Optics: Optical Society of America) p 809014

[70] Li L, Maslov K, Ku G and Wang L V 2009 Three-dimensional combined photoacoustic and optical coherence microscopy for in vivo microcirculation studies Optics express 17 16450-5

[71] Liu T, Wei Q, Song W, Burke J M, Jiao S and Zhang H F 2012 Near-infrared light photoacoustic ophthalmoscopy Biomedical optics express 3 792-9 
[72] Izatt J A, Kulkarni M D, Yazdanfar S, Barton J K and Welch A J 1997 In vivo bidirectional color Doppler flow imaging of picoliter blood volumes using optical coherence tomography Optics letters 22 1439-41

[73] Song W, Wei Q, Liu W, Liu T, Yi J, Sheibani N, Fawzi A A, Linsenmeier R A, Jiao S and Zhang H F 2014 A combined method to quantify the retinal metabolic rate of oxygen using photoacoustic ophthalmoscopy and optical coherence tomography Scientific reports 4

[74] Wang L V and Wu H-i 2012 Biomedical optics: principles and imaging: John Wiley \& Sons)

[75] Zhang X, Zhang H F and Jiao S 2012 Optical coherence photoacoustic microscopy: accomplishing optical coherence tomography and photoacoustic microscopy with a single light source Journal of biomedical optics 17 0305021-3

[76] Lee C, Han S, Kim S, Jeon M, Jeon M Y, Kim C and Kim J 2013 Combined photoacoustic and optical coherence tomography using a single near-infrared supercontinuum laser source Applied optics 52 1824-8

[77] Jacques S L Melanosome absorption coefficient.

[78] Vogel A and Birngruber R 1992 Temperature profiles in human retina and choroid during laser coagulation with different wavelengths ranging from 514 to $810 \mathrm{~nm}$ Lasers Light Ophthalmol 5 9-16

[79] Sarna T 1992 New trends in photobiology: Properties and function of the ocular melanin—a photobiophysical view Journal of Photochemistry and Photobiology B: Biology 12 215-58

[80] Korytowski W, Kalyanaraman B, Menon I, Sarna T and Sealy R C 1986 Reaction of superoxide anions with melanins: electron spin resonance and spin trapping studies Biochimica et Biophysica Acta (BBA)-General Subjects 882 145-53

[81] Delori F o C, Goger D G and Dorey C K 2001 Age-related accumulation and spatial distribution of lipofuscin in RPE of normal subjects Investigative ophthalmology \& visual science 42 1855-66

[82] Weiter J, Delori F, Wing G and Fitch K 1986 Retinal pigment epithelial lipofuscin and melanin and choroidal melanin in human eyes Investigative ophthalmology \& visual science 27 145-52

[83] Liu X, Wang C H, Dai C, Camesa A, Zhang H F and Jiao S 2013 Effect of contact lens on optical coherence tomography imaging of rodent retina Curr Eye Res 38 1235-40

[84] Ruggeri M, Wehbe H, Jiao S, Gregori G, Jockovich M E, Hackam A, Duan Y and Puliafito C A 2007 In vivo three-dimensional high-resolution imaging of rodent retina 
with spectral-domain optical coherence tomography Investigative ophthalmology \& visual science 48 1808-14

[85] Kim K H, Puoris' haag M, Maguluri G N, Umino Y, Cusato K, Barlow R B and de Boer J F 2008 Monitoring mouse retinal degeneration with high-resolution spectraldomain optical coherence tomography Journal of vision 8 17-

[86] Fischer M D, Huber G, Beck S C, Tanimoto N, Muehlfriedel R, Fahl E, Grimm C, Wenzel A, Remé C E and van de Pavert S A 2009 Noninvasive, in vivo assessment of mouse retinal structure using optical coherence tomography PloS one 4 e7507

[87] Xu J, Molday L L, Molday R S and Sarunic M V 2009 In vivo imaging of the mouse model of $\mathrm{X}$-linked juvenile retinoschisis with fourier domain optical coherence tomography Investigative ophthalmology \& visual science $\mathbf{5 0} 2989-93$

[88] Ruggeri M, Tsechpenakis G, Jiao S, Jockovich M E, Cebulla C, Hernandez E, Murray T G and Puliafito C A 2009 Retinal tumor imaging and volume quantification in mouse model using spectraldomain optical coherence tomography Optics express 17 4074-83

[89] Shousha M A, Perez V L, Wang J, Ide T, Jiao S, Chen Q, Chang V, Buchser N, Dubovy S R and Feuer W 2010 Use of ultra-high-resolution optical coherence tomography to detect in vivo characteristics of Descemet's membrane in Fuchs' dystrophy Ophthalmology 117 1220-7

[90] Pennesi M E, Michaels K V, Magee S S, Maricle A, Davin S P, Garg A K, Gale M J, Tu D C, Wen Y, Erker L R and Francis P J 2012 Long-Term Characterization of Retinal Degeneration in rd1 and rd10 Mice Using Spectral Domain Optical Coherence Tomography Investigative Ophthalmology \& Visual Science 53 4644-56

[91] Liang J, Williams D R and Miller D T 1997 Supernormal vision and highresolution retinal imaging through adaptive optics Josa a 14 2884-92

[92] Roorda A, Romero-Borja F, Donnelly W J, Queener H, Hebert T J and Campbell M C W 2002 Adaptive optics scanning laser ophthalmoscopy Optics Express 10 405-12

[93] Porter J, Queener H, Lin J, Thorn K and Awwal A 2006 Adaptive optics for vision science: Wiley Online Library)

[94] Geng Y, Greenberg K P, Wolfe R, Gray D C, Hunter J J, Dubra A, Flannery J G, Williams D R and Porter J 2009 In vivo imaging of microscopic structures in the rat retina Investigative ophthalmology \& visual science $505872-9$

[95] Barbero S, Marcos S and Jimenez-Alfaro I 2003 Optical aberrations of intraocular lenses measured in vivo and in vitro Journal of the Optical Society of America a-Optics Image Science and Vision 20 1841-51 
[96] Burns S A, Zhou Y, Lin C P, Bifano T G, Veilleux I and Webb R H 2004 Retinal imaging and wavefront sensing in mice Investigative Ophthalmology \& Visual Science 45 U1003-U

[97] de la Cera E G, Rodriguez G, Llorente L, Schaeffel F and Marcos S 2006 Optical aberrations in the mouse eye Vision Research 46 2546-53

[98] Srinivasan V J, Ko T H, Wojtkowski M, Carvalho M, Clermont A, Bursell S-E, Song Q H, Lem J, Duker J S, Schuman J S and Fujimoto J G 2006 Noninvasive volumetric Imaging and morphometry of the rodent retina with high-speed, ultrahighresolution optical coherence tomography Investigative Ophthalmology \& Visual Science $475522-8$

[99] Ridder W H, Nusinowitz S and Heckenlively J R 2002 Causes of cataract development in anesthetized mice Experimental Eye Research 75 365-70

[100] Kawaguchi I, Higashide T, Ohkubo S, Takeda H and Sugiyama K 2006 In vivo imaging and quantitative evaluation of the rat retinal nerve fiber layer using scanning laser ophthalmoscopy Investigative Ophthalmology \& Visual Science 47 2911-6

[101] Huber G, Beck S C, Grimm C, Sahaboglu-Tekgoz A, Paquet-Durand F, Wenzel A, Humphries P, Redmond T M, Seeliger M W and Fischer M D 2009 Spectral domain optical coherence tomography in mouse models of retinal degeneration Investigative ophthalmology \& visual science 50 5888-95

[102] Bartsch D, Zinser G and Freeman W R 1994 Resolution improvement in confocal scanning laser tomography of the human fundus Vision Science and its applications: technical digest (OSA, Washington, DC) 134-7

[103] American National Standard for Safe Use of Lasers, ANSI Z136.1. 2007.

[104] Wehbe H, Ruggeri M, Jiao S, Gregori G, Puliafito C A and Zhao W 2007 Automatic retinal blood flow calculation using spectral domain optical coherence tomography Optics Express 15 15193-206

[105] Weber A, Cheney M C, Smithwick Q Y and Elsner A E 2004 Polarimetric imaging and blood vessel quantification Optics Express 12 5178-90

[106] Liu X, Liu T, Wen R, Li Y, Puliafito C A, Zhang H F and Jiao S 2015 Optical coherence photoacoustic microscopy for in vivo multimodal retinal imaging Optics letters 40 1370-3

[107] Liu X, Wen R, Li Y and Jiao S 2016 Optical coherence photoacoustic microscopy (OC-PAM) with an intensity-modulated continuous-wave broadband light source Journal of Optics 18064001 
[108] Liu T, Liu X, Wen R, Lam B L and Jiao S 2015 In vivo imaging rhodopsin distribution in the photoreceptors with nano-second pulsed scanning laser ophthalmoscopy Quantitative imaging in medicine and surgery 563

[109] Liu T, Wen R, Lam B L, Puliafito C A and Jiao S 2015 Depth-resolved rhodopsin molecular contrast imaging for functional assessment of photoreceptors Scientific reports 5

[110] Murray T and Balogun O 2004 High-sensitivity laser-based acoustic microscopy using a modulated excitation source Applied physics letters 85 2974-6

[111] Maslov K and Wang L V 2008 Photoacoustic imaging of biological tissue with intensity-modulated continuous-wave laser Journal of biomedical optics 13 024006--5

[112] Yang X, Skrabalak S E, Li Z-Y, Xia Y and Wang L V 2007 Photoacoustic tomography of a rat cerebral cortex in vivo with Au nanocages as an optical contrast agent Nano letters 7 3798-802

[113] Wang Y, Xie X, Wang X, Ku G, Gill K L, O'Neal D P, Stoica G and Wang L V 2004 Photoacoustic tomography of a nanoshell contrast agent in the in vivo rat brain Nano Letters 4 1689-92

[114] Eghtedari M, Oraevsky A, Copland J A, Kotov N A, Conjusteau A and Motamedi M 2007 High sensitivity of in vivo detection of gold nanorods using a laser optoacoustic imaging system Nano letters 7 1914-8

[115] Kim K, Huang S-W, Ashkenazi S, O’Donnell M, Agarwal A, Kotov N A, Denny M F and Kaplan M J 2007 Photoacoustic imaging of early inflammatory response using gold nanorods Applied physics letters $\mathbf{9 0} 223901$

[116] Song K H, Kim C, Maslov K and Wang L V 2009 Noninvasive in vivo spectroscopic nanorod-contrast photoacoustic mapping of sentinel lymph nodes European journal of radiology 70 227-31

[117] Eghtedari M, Liopo A V, Copland J A, Oraevsky A A and Motamedi M 2008 Engineering of hetero-functional gold nanorods for the in vivo molecular targeting of breast cancer cells Nano letters 9 287-91

[118] Agarwal A, Huang S, O’Donnell M, Day K, Day M, Kotov N and Ashkenazi S 2007 Targeted gold nanorod contrast agent for prostate cancer detection by photoacoustic imaging Journal of applied physics 102064701

[119] Calasso I G, Craig W and Diebold G J 2001 Photoacoustic point source Physical Review Letters 863550

[120] Chen Z, Milner T E, Dave D and Nelson J S 1997 Optical Doppler tomographic imaging of fluid flow velocity in highly scattering media Optics letters 22 64-6 
[121] Barton J K and Stromski S 2005 Flow measurement without phase information in optical coherence tomography images Optics express 13 5234-9

[122] Schmitt J, Xiang S and Yung K 1998 Differential absorption imaging with optical coherence tomography JOSA A 15 2288-96

[123] Mariampillai A, Standish B A, Moriyama E H, Khurana M, Munce N R, Leung M K, Jiang J, Cable A, Wilson B C and Vitkin I A 2008 Speckle variance detection of microvasculature using swept-source optical coherence tomography Optics letters 33 $1530-2$

[124] Driver I, Feather J, King P and Dawson J 1989 The optical properties of aqueous suspensions of Intralipid, a fat emulsion Physics in medicine and biology 341927

[125] Flock S T, Jacques S L, Wilson B C, Star W M and van Gemert M J 1992 Optical properties of Intralipid: a phantom medium for light propagation studies Lasers in surgery and medicine 12 510-9

[126] Giusto A, Saija R, Iatì M A, Denti P, Borghese F and Sindoni O I 2003 Optical properties of high-density dispersions of particles: application to intralipid solutions Applied optics 42 4375-80

[127] Michels R, Foschum F and Kienle A 2008 Optical properties of fat emulsions Optics Express 16 5907-25

[128] Van Staveren H J, Moes C J, van Marie J, Prahl S A and Van Gemert M J 1991 Light scattering in lntralipid-10\% in the wavelength range of 400-1100 nm Applied optics 30 4507-14

[129] Jacques S L Optical properties of "Intralipid".

[130] Jacques S L 2013 Optical properties of biological tissues: a review Physics in medicine and biology 58 R37

[131] Tseng S-H, Grant A and Durkin A J 2008 In vivo determination of skin nearinfrared optical properties using diffuse optical spectroscopy Journal of biomedical optics 13 014016--7

[132] Jacques S L, Glickman R D and Schwartz J A 1996 Internal absorption coefficient and threshold for pulsed laser disruption of melanosomes isolated from retinal pigment epithelium. In: Photonics West'96: International Society for Optics and Photonics) pp 468-77

[133] Bashkatov A N, Genina E A, Kochubey V I, Stolnitz M M, Bashkatova T A, Novikova O V, Peshkova A Y and Tuchin V V 2000 Optical properties of melanin in the skin and skinlike phantoms. In: EOS/SPIE European Biomedical Optics Week:

International Society for Optics and Photonics) pp 219-26 
[134] Hammer M, Roggan A, Schweitzer D and Muller G 1995 Optical properties of ocular fundus tissues-an in vitro study using the double-integrating-sphere technique and inverse Monte Carlo simulation Physics in medicine and biology 40963 


\section{VITA}

\section{XIAOJING LIU}

Born, Wuhan, China

2006-2010

Bachelor of Science in Biomedical Engineering Huazhong University of Science and Technology Wuhan, China

2010-2012

Master of Science in Medical Device and Diagnostic Engineering University of Southern California

Los Angeles, California

2012-2017

Doctoral Candidate, Research Assistant, Teaching Assistant Florida International University

Miami, Florida

2015

The paper of "Optical coherence photoacoustic microscopy for in vivo multimodal retinal imaging" is honored to be "Feature of The Week" on OCT News home page

\section{PUBLICATIONS AND PRESENTATIONS}

Liu, X., Wen, R., Li, Y., \& Jiao, S. (2016). Optical coherence photoacoustic microscopy (OC-PAM) with an intensity-modulated continuous-wave broadband light source. Journal of Optics, 18(6), 064001.

Liu, X., Liu, T., Wen, R., Li, Y., Puliafito, C. A., Zhang, H. F., \& Jiao, S. (2015). Optical coherence photoacoustic microscopy for in vivo multimodal retinal imaging. Optics letters, 40(7), 1370-1373.

Liu, X., (April, 2015). Optical coherence photoacoustic microscopy (OC-PAM) for in vivo multimodal retinal imaging. Poster presented at 2015 International Year of LightPhotonics in Healthcare, Miami, Florida.

Liu, X., (February, 2015). Optical coherence photoacoustic microscopy (OC-PAM) for in vivo multimodal retinal imaging. Poster presented at SPIE Photonics West 2015, San Francisco, California.

Jiang, M., Liu, T., Liu, X., \& Jiao, S. (2014). Simultaneous optical coherence tomography and lipofuscin autofluorescence imaging of the retina with a single broadband light source at 480nm. Biomedical optics express, 5(12), 4242-4248. 
Liu, T., Liu, X., Wen, R., Lam, B. L., \& Jiao, S. (2014). In vivo imaging rhodopsin distribution in the photoreceptors with nano-second pulsed scanning laser ophthalmoscopy. Quantitative imaging in medicine and surgery, 5(1), 63-68.

Liu, X., (February, 2014). Absolute retinal blood flow measurement with a dual-beam Doppler OCT. Paper presented at SPIE Photonics West 2014, San Francisco, California.

Liu, X., (February, 2014). Effect of contact lens on optical coherence tomography imaging of rodent retina. Poster presented at SPIE Photonics West 2014, San Francisco, California.

Liu, X., Wang, C. H., Dai, C., Camesa, A., Zhang, H. F., \& Jiao, S. (2013). Effect of contact lens on optical coherence tomography imaging of rodent retina. Current eye research, 38(12), 1235-1240.

Dai, C., Liu, X., Zhang, H. F., Puliafito, C. A., \& Jiao, S. (2013). Absolute Retinal Blood Flow Measurement with a Dual-Beam Doppler Optical Coherence Tomography Imaging Retinal Blood Flow with Dual-Beam OCT. Investigative ophthalmology \& visual science, 54(13), 7998-8003.

Liu, X., (May, 2013). Simultaneous optical coherence tomography and autofluorescence microscopy with a single light source. Paper presented at the 29th Southern Biomedical Engineering Conference, Miami, Florida.

Liu, X., (February, 2013). Laser-scanning photoacoustic microscopy with ultrasonic phased array transducer. Paper presented at SPIE Photonics West 2013, San Francisco, California.

Dai, C., Liu, X., \& Jiao, S. (2012). Simultaneous optical coherence tomography and autofluorescence microscopy with a single light source. Journal of biomedical optics, 17(8), 0805021-0805023. 